Review

\title{
Cytoreductive surgery plus hyperthermic intraperitoneal chemotherapy improves survival for peritoneal carcinomatosis from colorectal cancer: a systematic review and meta-analysis of current evidence
}

\author{
Chao-Qun Huang ${ }^{1, *}$, Yao Min ${ }^{2, *}$, Shu-Yi Wang ${ }^{1}$, Xiao-Jun Yang1, Yang Liu ${ }^{3}$, Bin \\ Xiong ${ }^{1}$, Yutaka Yonemura ${ }^{3}$ and Yan $\mathbf{L i}^{1,4}$ \\ ${ }^{1}$ Department of Gastrointestinal Surgery, Zhongnan Hospital of Wuhan University, Hubei Cancer Clinical Study Center \& \\ Hubei Key Laboratory of Tumor Biological Behaviors, Wuhan Clinical Research Center for Peritoneal Carcinomatosis, Wuhan, \\ P.R. China \\ 2 Department of Ophthalmology, Central Hospital of Wuhan Affiliated to Tongji Medical College of Huazhong University of \\ Science and Technology, Wuhan, P.R. China \\ ${ }^{3}$ NPO to Support Peritoneal Surface Malignancy Treatment, Osaka, Japan \\ ${ }^{4}$ Department of Peritoneal Cancer Surgery, Beijing Shijitan Hospital of the Capital Medical University, Beijing, P.R. China \\ * These authors have contributed equally to this work
}

Correspondence to: Yan Li, email: liyansd2@163.com

Keywords: colorectal cancer; peritoneal carcinomatosis; cytoreductive surgery; hyperthermic intraperitoneal chemotherapy; meta-analysis

Received: August 29, $2016 \quad$ Accepted: January 24, $2017 \quad$ Published: April 27, 2017

Copyright: Huang et al. This is an open-access article distributed under the terms of the Creative Commons Attribution License 3.0 (CC BY 3.0), which permits unrestricted use, distribution, and reproduction in any medium, provided the original author and source are credited.

\section{ABSTRACT}

Objectives The therapeutic efficacy of cytoreductive surgery (CRS) plus hyperthermic intraperitoneal chemotherapy (HIPEC) for patients with peritoneal carcinomatosis (PC) from colorectal cancer (CRC) is still under debate. This metaanalysis and systematic review of published literature on this comprehensive strategy aims to evaluate its efficacy on CRC patients with PC.

Methods A systemic review with meta-analysis of published literatures on treatment of CRS plus HIPEC for patients with PC from CRC was performed. In addition, a summary of study results of published literatures concerning CRS plus HIPEC treating patients with PC from CRC was also conducted.

Results A total of 76 studies were selected, including 1 randomized controlled trial, 14 non-randomized controlled studies, and 61 non-controlled studies. The pooled hazard ratios (HRs) for overall survival (OS) in the 15 researches for meta-analysis was 2.67 (95\% CI, 2.21-3.23, $\left.I^{2}=0 \%, P<0.00001\right)$, and no significant evidence of publication bias was found. The difference of chemotherapy regimens of HIPEC was not associated with OS and DFS (disease-free survival) after CRS and HIPEC, with no significant difference of heterogeneity $\left(P=0.27, I^{2}=24.1 \%\right)$. In both groups of mitomycin C based HIPEC group and oxaliplatin group, patients received HIPEC had significant better survival $(P<\mathbf{0 . 0 0 0 0 1})$. The mean mortality and morbidity for HIPEC program were $2.8 \%$ and $33.0 \%$, respectively.

Conclusions This meta-analysis revealed that comprehensive therapeutic strategy of CRS plus HIPEC could bring survival benefit for selected patients with PC from CRC with acceptable safety.

\section{INTRODUCTION}

Peritoneal carcinomatosis (PC), as a lethal regional progression for patients with colorectal cancer (CRC), has long been considered as a terminal condition with few effective treatments. In the past, the median overall survival (OS) of PC from colorectal cancer is 4 to 7 months after palliative surgery or 5-FU-based systemic 
chemotherapy with best supportive care [1-3]. Current systemic chemotherapy focusing on new chemotherapeutic agents such as oxaliplatin and irinotecan, along with antiangiogenesis molecular targeting agents cetuximab and bevacizumab [4-7], could extend the median OS up to about 12 months [5]. However, long-term survival is still hard to be achieved by systemic chemotherapy alone.

Researches on treatment of CRC PC did not reveal promising progress until the development of a comprehensive treatment strategy including cytoreductive surgery (CRS) plus hyperthermic intraperitoneal chemotherapy (HIPEC) and perioperative chemotherapy. [8-15] This new comprehensive treatment improves the median OS of selected patients with CRC PC up to 2163 months, and 5-year survival rate up to approximately $40 \%$ [16-28], or even 58\% according to the American Society of Peritoneal Surface Malignancies (ASPSM) multi-institution study [29]. It has been widely recognized in North America, Europe, Australia, and Japan [14, 24, 26, 30-32]. In the 9th International Congress on Peritoneal Surface Malignancies in Amsterdam in 2014, peritoneal surface oncology group international (PSOGI) reached a consensus that CRS+HIPEC should be considered as the standard therapy for the selected patients with mild-tomoderate CRC PC [33].
Nevertheless, therapeutic efficacy of this comprehensive treatment strategy for CRC PC patient remains controversial due to insufficient convincing evidence. Therefore, we conducted this meta-analysis of published clinical studies to verify the efficacy of this strategy against CRC PC.

\section{RESULTS}

\section{Basic characteristics of all data}

\section{Results of literature search}

Literature search identified 326 researches, 76 of which met the inclusion criteria, including 1 randomized controlled trail (RCT) (87 patients) [12], 14 nonrandomized controlled studies (3,092 patients) [13-15, 26, $28,29,34-40,99]$, and 61 non-controlled studies $(6,857$ patients) $[16,19-21,41-92,100-104]$. The other 250 studies were excluded for miscellaneous reasons, and the flowchart of search strategy is showed in Figure 1. We conducted a meta-analysis on the 15 controlled studies $(3,179$ patients $)$ and a summary of 76 HIPEC-related studies (10,036 patients).

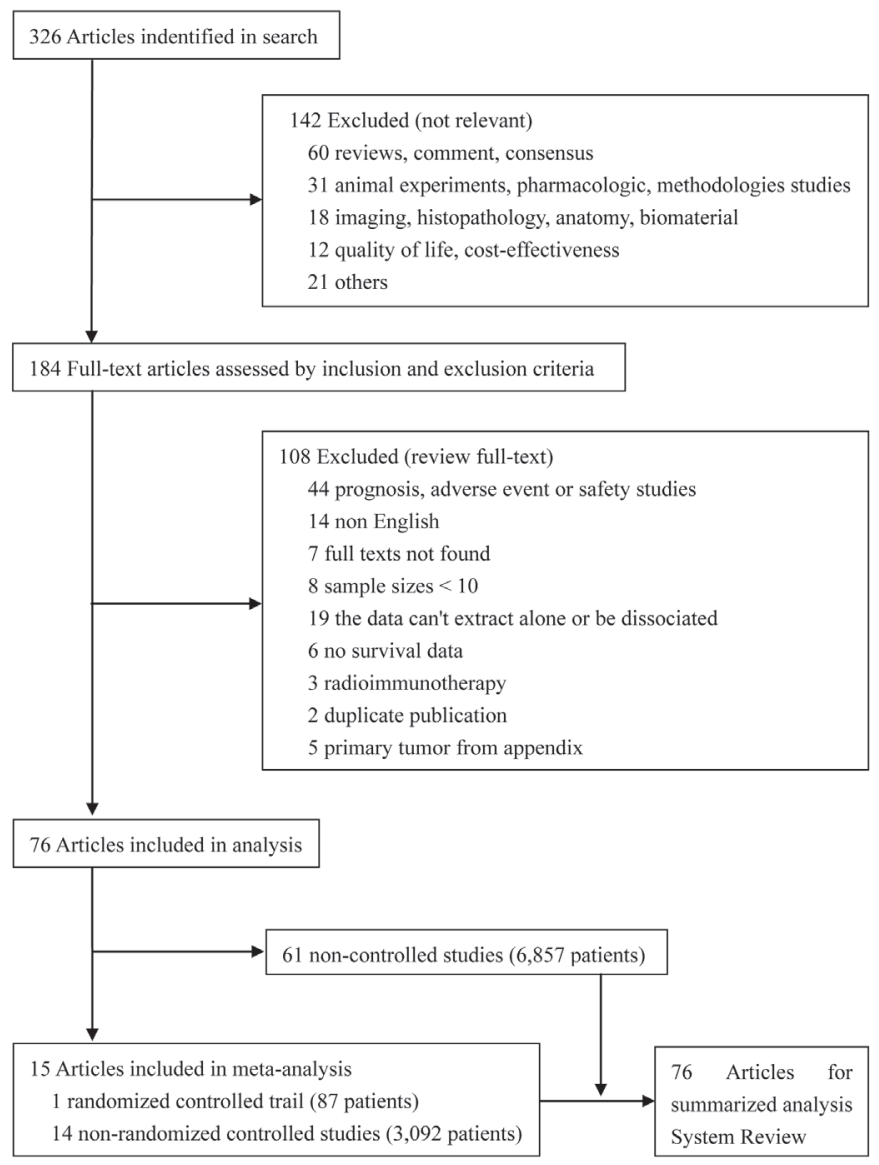

Figure 1: Study flowchart of systematic reviews and meta-analyses. 
Table 1: Major Characteristics of Fifteen Controlled Researches on Peritoneal Carcinomatosis (PC) from Colorectal Cancer (CRC) Treated with Cytoreductive Surgery (CRS) plus Hyperthermic Intraperitoneal Chemotherapy (HIPEC) versus Surgery alone with Systemic Chemotherapy (SC) and/or Early Postoperative Intraperitoneal Chemotherapy (EPIC).

\begin{tabular}{|c|c|c|c|c|c|c|c|}
\hline \multirow{2}{*}{ Author/ Year/ Country } & \multirow{2}{*}{\begin{tabular}{|l|} 
Participating \\
Institutions
\end{tabular}} & \multirow{2}{*}{\begin{tabular}{|l|} 
Study \\
Period
\end{tabular}} & \multirow{2}{*}{ Design } & \multirow{2}{*}{\begin{tabular}{|l} 
Level of \\
Evidence
\end{tabular}} & \multirow{2}{*}{$\begin{array}{|ll|}\text { Number } & \text { of } \\
\text { CRC PC } & \end{array}$} & \multicolumn{2}{|l|}{ Treatment strategy } \\
\hline & & & & & & HIPEC group & Control group \\
\hline $\begin{array}{l}\text { Chua TC/ 2009/ Australia } \\
\text { [34] }\end{array}$ & 2 & $1997-2008$ & retrospective & $\mathrm{IIb}$ & $15(15 / 33)$ & $\begin{array}{l}\text { CRS+HIPEC } 7 \mathrm{pts} ; \\
\text { HIPEC: MMC }\left(10-20 \mathrm{mg} / \mathrm{m}^{2}\right) \\
\text { for } 90 \text { min at } 42^{\circ} \mathrm{C} \text { using the } \\
\text { closed abdomen technique. } \\
\text { No EPIC. } \\
\text { SC: FOLFOX and } \\
\text { Bevacizumab }\end{array}$ & $\begin{array}{l}\text { SC } 8 \text { pts } \\
\text { SC: FOLFOX and } \\
\text { Bevacizumab } \\
\text { No HIPEC } \\
\text { No EPIC }\end{array}$ \\
\hline $\begin{array}{l}\text { Chua TC/ 2011/ Australia } \\
\text { [15] }\end{array}$ & 3 & $1988-2009$ & retrospective & IIa & $294(294 / 294)$ & $\begin{array}{l}\text { CRS+HIPEC+SC } 110 \mathrm{pts} \\
\text { HIPEC: MMC }\left(10-20 \mathrm{mg} / \mathrm{m}^{2}\right) \\
\text { for } 90 \mathrm{~min} \text { at } 42^{\circ} \mathrm{C} \text { using the } \\
\text { Coliseum technique. } \\
\text { No EPIC } \\
\text { SC: } 5 \text {-FU + LV; 5-FU + LV or } \\
\text { CBP with L-OHP or CPT-11; } \\
\text { or Regimen 2 + BEV, C225, } \\
\text { or PAN }\end{array}$ & $\begin{array}{l}\text { Surgery and/or SC } \\
184 \text { pts } \\
\text { SC: 5-FU + LV; } \\
5 \text {-FU + LV or } \\
\text { CBP with L-OHP } \\
\text { or CPT-11; or } \\
\text { Regimen 2 + BEV, } \\
\text { C225, or PAN } \\
\text { No EPIC } \\
\text { No HIPEC }\end{array}$ \\
\hline $\begin{array}{l}\text { Chua TC/ 2013/ Australia } \\
\text { [26] }\end{array}$ & 1 & $1996-2011$ & prospective & IIa & $75(75 / 98)$ & $\begin{array}{l}\text { CRS+HIPEC with/without } \\
\text { EPIC 75pts } \\
\text { HIPEC: MMC }(10-12.5 \mathrm{mg} / \\
\left.\mathrm{m}^{2}\right) \text { or L-OHP }\left(460 \mathrm{mg} / \mathrm{m}^{2}\right) \\
\text { for } 90 \mathrm{~min} \text { at } 42^{\circ} \mathrm{C} \text { using the } \\
\text { closed abdomen technique; } \\
\text { Before starting HIEPC, } \\
\text { oxaliplatin, 5-FU }(400 \mathrm{mg} / \\
\left.\mathrm{m}^{2}\right) \text { and LV }\left(20 \mathrm{mg} / \mathrm{m}^{2}\right) \mathrm{by} \\
\text { intravenous perfusion. } \\
\text { EPIC: 5-FU (650-800 mg/ } \\
\left.\mathrm{m}^{2} / \mathrm{d}\right) \text { on Day } 1-5 \text { after surgery } \\
\mathrm{SC}(\text { not reported) }\end{array}$ & $\begin{array}{l}\text { EPIC alone } 23 \mathrm{pts} \\
\text { EPIC: } 5-\mathrm{FU}(650- \\
\left.800 \mathrm{mg} / \mathrm{m}^{2} / \mathrm{d}\right) \\
\text { on Day } 1-5 \text { after } \\
\text { surgery } \\
\text { SC (not reported) } \\
\text { No HIPEC }\end{array}$ \\
\hline
\end{tabular}

Note: CRC: colorectal cancer; PC: peritoneal carcinomatosis; Pts: patients; MMC: mitomycin C; DDP: cisplatin; FU: fluorouracil; LV: leucovorin; L-OHP: oxaliplatin; CPT-11: irinotecan; Cap: capecitabine; C225: cetuximab; CPT: camptothecin; BEV: bevacizumab; DXL: docetaxel; CBP: carboplatin; PAN: panitumumab;

Table 2: Major Characteristics of Fifteen Controlled Researches on Peritoneal Carcinomatosis (PC) from Colorectal Cancer (CRC) Treated with Cytoreductive Surgery (CRS) plus Hyperthermic Intraperitoneal Chemotherapy (HIPEC) versus Surgery alone with Systemic Chemotherapy (SC) and/or Early Postoperative Intraperitoneal Chemotherapy (EPIC).

\begin{tabular}{|c|c|c|c|c|c|c|c|}
\hline \multirow{2}{*}{ Author/ Year/ Country } & \multirow{2}{*}{$\begin{array}{l}\text { Participating } \\
\text { Institutions }\end{array}$} & \multirow{2}{*}{$\begin{array}{l}\text { Study } \\
\text { Period }\end{array}$} & \multirow{2}{*}{ Design } & \multirow{2}{*}{$\begin{array}{l}\text { Level of } \\
\text { Evidence }\end{array}$} & \multirow{2}{*}{\begin{tabular}{|l|l|}
$\begin{array}{l}\text { Number } \\
\text { of }\end{array}$ & CRC \\
PC
\end{tabular}} & \multicolumn{2}{|l|}{ Treatment strategy } \\
\hline & & & & & & HIPEC group & Control group \\
\hline Elias D/ 2001/ France [35] & 1 & 1993-1999 & prospective & IIa & $55(55 / 64)$ & $\begin{array}{l}\text { HIPEC } 27 \mathrm{pts} \\
\text { HIPEC: } 1 . \text { MMC }^{\circ}(5,8, \text { or } 10 \mathrm{mg} / \mathrm{L}) \text { for } 1 \mathrm{~h} \\
\text { between } 41^{\circ} \mathrm{C} \text { and } 44^{\circ} \mathrm{C} \text { using the Coliseum } \\
\text { technique. } 2 \text {. MMC }\left(20 \mathrm{mg} / \mathrm{m}^{2}\right)+\text { DDP }(200 \\
\left.\mathrm{mg} / \mathrm{m}^{2}\right) \text { for } 1 \mathrm{~h} \text { between } 41^{\circ} \mathrm{C} \text { and } \\
44^{\circ} \mathrm{C} \text { using the Coliseum technique. } \\
\text { EPIC: MMC }\left(10 \mathrm{~g} / \mathrm{m}^{2}\right) \text { on Day } 1+5 \text {-FU }(500 \\
\left.\mathrm{mg} / \mathrm{m}^{2}\right) \text { form Day } 2 \text { to Day } 6 \text { lasted } 23 \mathrm{~h} \\
\text { No SC }\end{array}$ & $\begin{array}{l}\text { EPIC37 pts } \\
\text { EPIC: MMC }(10 \\
\left.\mathrm{g} / \mathrm{m}^{2}\right) \text { on Day } 1+ \\
5 \text {-FU }\left(500 \mathrm{mg} / \mathrm{m}^{2}\right) \\
\text { form Day } 2 \text { to Day } \\
6 \text { lasted } 23 \mathrm{~h} \\
\text { No HIPEC } \\
\text { No SC }\end{array}$ \\
\hline Elias D/ 2007/ France [36] & 1 & \begin{tabular}{|l}
$1999-2002$ \\
$1994-2000$
\end{tabular} & prospective & IIa & $46(46 / 46)$ & $\begin{array}{l}\text { CRS+HIPEC } 23 \mathrm{pts} \\
\text { HIPEC: L-OHP }\left(460 \mathrm{mg} / \mathrm{m}^{2}\right) \text { for } 35 \mathrm{~min} \\
\text { between } 42-44^{\circ} \mathrm{C} \text { using the Coliseum } \\
\text { technique; Before starting HIEPC, 5-FU }(400 \\
\left.\mathrm{mg} / \mathrm{m}^{2}\right) \text { and } \mathrm{LV}\left(20 \mathrm{mg} / \mathrm{m}^{2}\right) \text { by intravenous } \\
\text { perfusion. } \\
\text { EPIC: } \mathrm{MMC}\left(10 \mathrm{mg} / \mathrm{m}^{2}\right) \text { at day } 0 \text {, then } 5-\mathrm{FU} \\
\left(650 \mathrm{mg} / \mathrm{m}^{2}\right) \text { for the next } 4 \text { days } \\
\text { SC }(\text { not reported) }\end{array}$ & $\begin{array}{l}\text { EPIC } 23 \text { pts } \\
\text { EPIC: MMC (10 } \\
\left.\mathrm{mg} / \mathrm{m}^{2}\right) \text { at day } 0, \\
\text { then 5-FU ( } 650 \\
\left.\mathrm{mg} / \mathrm{m}^{2}\right) \text { for the next } \\
4 \text { days } \\
\text { SC (not reported) } \\
\text { No HIPEC }\end{array}$ \\
\hline Elias D/ 2009/ France [28] & $\begin{array}{l}6 \\
\text { (Only one } \\
\text { centre } \\
\text { conducted } \\
\text { HIPEC, the } \\
\text { rest of } 5 \text { as a } \\
\text { control) }\end{array}$ & $1998-2003$ & retrospective & IIa & $96(96 / 96)$ & $\begin{array}{l}\text { Neoadjuvant IPC+CRS+HIPEC+SC } 48 \text { pts } \\
\text { Neoadjuvant IPC: } \mathrm{L}-\mathrm{OHP} \text { or CPT-11 (not } \\
\text { reported the detailed regimen) } \\
\text { HIPEC: L-OHP }\left(460 \mathrm{mg} / \mathrm{m}^{2}\right) \text { over } 30 \mathrm{~min} \text { at } \\
43^{\circ} \mathrm{C} \text { using the Coliseum technique. Before } \\
\text { starting HIEPC, } 5 \text {-FU } 400 \mathrm{mg} / \mathrm{m}^{2} \text { and LV } 20 \\
\mathrm{mg} / \mathrm{m}^{2} \text { by intravenous perfusion. } \\
\text { SC: } 1 \text {. FU Plus CPT-11 or L-OHP, LV; } 2 \text {. Cap } \\
\text { Plus L-OHP; 3. CPT-11 plus C } 225 \text { and CPT }\end{array}$ & $\begin{array}{l}\text { Surgery and/or SC } \\
48 \text { pts } \\
\text { SC: 1. FU Plus } \\
\text { CPT-11 or L-OHP, } \\
\text { LV; 2. Cap Plus } \\
\text { L-OHP; 3. CPT-11 } \\
\text { plus C225 and CPT } \\
\text { No HIPEC } \\
\text { No EPIC }\end{array}$ \\
\hline
\end{tabular}

Note: CRC: colorectal cancer; PC: peritoneal carcinomatosis; Pts: patients; MMC: mitomycin C; DDP: cisplatin; FU: fluorouracil; LV: leucovorin; L-OHP: oxaliplatin; CPT-11: irinotecan; Cap: capecitabine; C225: cetuximab; CPT: camptothecin; BEV: bevacizumab; DXL: docetaxel; CBP: carboplatin; PAN: panitumumab; 


\section{Study characteristics}

The characteristics of 15 controlled studies [8-15, 26, 28, 29, 34-40, 99] were shown in Table 1-5, and all 76 selected studies [12-16, 19-21, 26, 28, 29, 34-92, 99-104] were summarized in Table 6-10. All these studies were published between 1993 and 2016 as full texts, performed in 19 countries and regions (Table 11-19). Fifty-eight studies were single-center studies $[12,16,19,21,35,36$, 38-43, 45, 43-53, 55-57, 60-63, 66-71, 74-83, 86-92, 99, $100,102-104]$, and the other 18 were multicenter studies (participating institutions from 2 to 28) [13-15, 28, 29, 34, $37,44,46,54,58,59,64,65,72,73,84,85,101]$. In these multicenter studies, 6 studies were performed by over 10 participating institutions included studies conducted by Glehen et al ( $n=28$, a central database) [13], Glehen et al ( $n=25$, a central database) [54], Elias et al $(n=$ 25 , a central database) [14], Esquivel et al $(n=21$, The American Society of Peritoneal Surface Malignancies
$(\mathrm{ASPSM}))$ [29], and Prada-Villaverde et al $(n=15)$ [72]. A total of 63 articles were retrospective studies, in which 11 articles were included in this meta-analysis [13-16, 19-21, $28,29,34,37-40,43-48,50-52,54-57,59-66,68-72$, 74$88,91,99-104]$. Thirteen articles were prospective studies, in which 4 were included in this meta-analysis [12, 26, 35, $36,42,49,53,58,67,73,89,90,92]$. According to the North-England evidence-based guidelines [34, 35], there was one evidence level $\mathrm{Ib}$ in this meta-analysis [12], the rest cohort studies or "outcome" researches were evidence level II.[13-15, 26, 28, 29, 34-40, 99]

\section{Patients characteristics}

In this meta-analysis, the median complete cytoreduction (CC0-1) rate was $72.2 \%$ (range, 32.4\% $100 \%$ ), including 4 studies with $100 \%$ CC0 [28, 35, 36, 40], 7 studies with $50 \%$ - 99\% CC0 [14, 15, 26, 29, 34, $37,99]$, and 4 studies with $<50 \%$ CC0 [12, 13, 38, 39]. Major clinico-pathologic characteristics of the 6,857 CRC

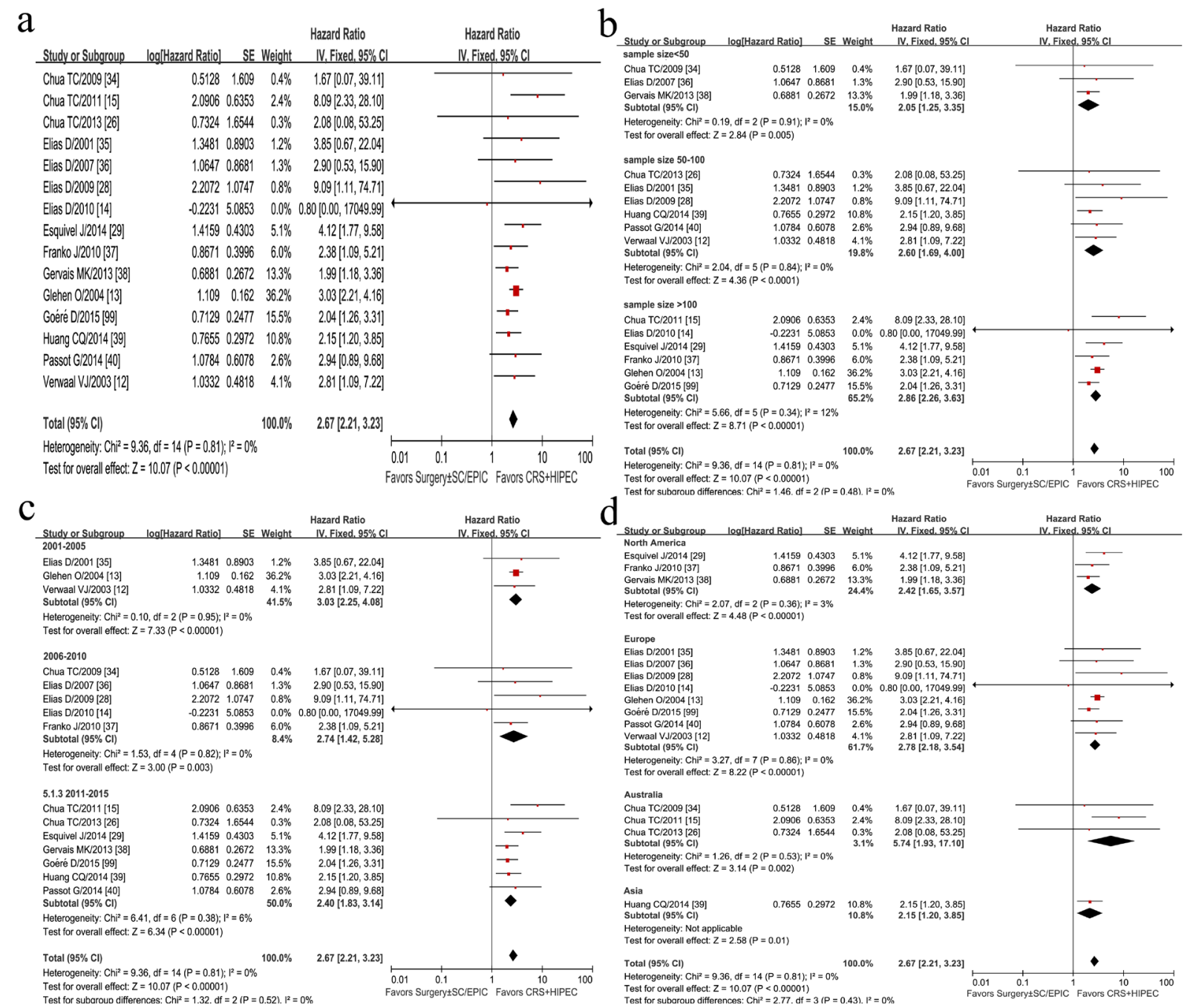

Figure 2: Forest plots of 15 studies displaying the results of the meta-analysis on hazard ratios (HR) for overall survival (OS) (a); Sensitivity analysis of sample size difference (b), published-time difference (c), and geographic-distribution difference (d). 
Table 3: Major Characteristics of Fifteen Controlled Researches on Peritoneal Carcinomatosis (PC) from Colorectal Cancer (CRC) Treated with Cytoreductive Surgery (CRS) plus Hyperthermic Intraperitoneal Chemotherapy (HIPEC) versus Surgery alone with Systemic Chemotherapy (SC) and/or Early Postoperative Intraperitoneal Chemotherapy (EPIC).

\begin{tabular}{|c|c|c|c|c|c|c|c|}
\hline \multirow{2}{*}{\begin{tabular}{|l} 
Author/ \\
Country
\end{tabular}} & \multirow{2}{*}{\begin{tabular}{|l|} 
Participating \\
Institutions
\end{tabular}} & \multirow{2}{*}{\begin{tabular}{|l|} 
Study \\
Period
\end{tabular}} & \multirow{2}{*}{ Design } & \multirow{2}{*}{$\begin{array}{l}\text { Level of } \\
\text { Evidence }\end{array}$} & \multirow{2}{*}{\begin{tabular}{|l|l|} 
Number \\
CRC PC
\end{tabular}} & \multicolumn{2}{|l|}{ Treatment strategy } \\
\hline & & & & & & HIPEC group & Control group \\
\hline $\begin{array}{l}\text { Elias D/2010/ France } \\
\text { [14] }\end{array}$ & $\begin{array}{l}25 \\
\text { (a } \quad \text { central } \\
\text { database) }\end{array}$ & $1990-2007$ & retrospective & IIa & $523(523 / 523)$ & $\begin{array}{l}\text { CRS+HIPEC with/without SC } \\
443 \text { pts } \\
\text { CRS+HIPEC+EPIC with/without } \\
\text { SC 9 pts } \\
\text { HIPEC: } 1 \text { MMC }\left(30-50 \mathrm{mg} / \mathrm{m}^{2}\right) \\
\pm \text { DDP }\left(50-100 \mathrm{mg} / \mathrm{m}^{2}\right) \text { during } \\
60 \text { to } 120 \mathrm{~min} \text { at } 41^{\circ} \mathrm{C} \text { using } \\
\text { Coliseum or closed abdomen } \\
\text { technique; 2. L-OHP }(360- \\
\left.460 \mathrm{mg} / \mathrm{m}^{2}\right) \pm C P T-11(200 \mathrm{mg} / \\
\left.\mathrm{m}^{2}\right)+ \text { intravenous } 5-\mathrm{FU} \text { and } \mathrm{LV} \\
\text { during } 30 \text { minutes at } 43^{\circ} \mathrm{C} \text { using } \\
\text { Coliseum or closed abdomen } \\
\text { technique. } \\
\text { EPIC: MMC }\left(10 \mathrm{~g} / \mathrm{m}^{2}\right) \text { on Day } \\
\text { 1+5-FU }\left(600 \mathrm{mg} / \mathrm{m}^{2}\right) \text { form Day } 2 \\
\text { to Day } 6 \text { lasted } 23 \mathrm{~h} \\
\text { SC: not reported the detailed } \\
\text { regimen }\end{array}$ & $\begin{array}{l}\text { CRS+EPIC with/ } \\
\text { without SC } 84 \mathrm{pts} \\
\text { EPIC: MMC (10 } \\
\left.\mathrm{g} / \mathrm{m}^{2}\right) \text { on Day } \\
1+5-\mathrm{FU}(600 \mathrm{mg} / \\
\left.\mathrm{m}^{2}\right) \text { form Day } 2 \text { to } \\
\text { Day } 6 \text { lasted } 23 \mathrm{~h} \\
\text { SC: not reported } \\
\text { the detailed } \\
\text { regimen } \\
\text { No HIPEC }\end{array}$ \\
\hline $\begin{array}{l}\text { Esquivel J/2014 / } \\
\text { America [29] }\end{array}$ & $\begin{array}{l}21 \\
\text { (The } \\
\text { American } \\
\text { Society of } \\
\text { Peritoneal } \\
\text { Surface } \\
\text { Malignancies } \\
\text { (ASPSM)) }\end{array}$ & $1985-2012$ & retrospective & IIa & $\begin{array}{l}1,013 \\
(1,013 / 1,013)\end{array}$ & $\begin{array}{l}\text { CRS+HIPEC } 705 \text { pts } \\
\text { HIPEC: The chemotherapy drugs } \\
\text { L-OHP or MMC or others but not } \\
\text { reported the remaining details. } \\
\text { SC (not detailed reported) } \\
\text { No EPIC }\end{array}$ & $\begin{array}{l}\text { SC alone } 308 \text { pts } \\
\text { SC (not detailed } \\
\text { reported) } \\
\text { No EPIC } \\
\text { No HIPEC }\end{array}$ \\
\hline $\begin{array}{l}\text { Franko J// 2010/ } \\
\text { America [37] }\end{array}$ & $\begin{array}{l}3 \\
\text { (one centre } \\
\text { conducted } \\
\text { HIPEC, two } \\
\text { centre as a } \\
\text { control) }\end{array}$ & 2001-2007 & retrospective & IIa & $105(105 / 105)$ & $\begin{array}{l}\text { CRS+HIPEC+SC } 67 \text { pts } \\
\text { HIPEC: MMC } 30 \mathrm{mg} \text { for the first } \\
\text { hour, followed by an additional } \\
10 \mathrm{mg} \text { for } 40 \text { more minutes using } \\
\text { the closed abdomen technique. } \\
\text { (Perfusion fluid temperature not } \\
\text { reported) } \\
\text { No EPIC } \\
\text { SC: } 1 \text {. 5-FU and CPT-11; 2. } \\
\text { L-OHP or biological agents (BEV } \\
\text { and/or C225) }\end{array}$ & $\begin{array}{l}\text { Surgery + SC } 38 \\
\text { pts } \\
\text { SC: 1. 5-FU and } \\
\text { CPT-11;2. L-OHP } \\
\text { or biological } \\
\text { agents (BEV and// } \\
\text { or C225) } \\
\text { No EPIC } \\
\text { No HIPEC }\end{array}$ \\
\hline
\end{tabular}

Note: CRC: colorectal cancer; PC: peritoneal carcinomatosis; Pts: patients; MMC: mitomycin C; DDP: cisplatin; FU: fluorouracil; LV: leucovorin; L-OHP: oxaliplatin; CPT-11: irinotecan; Cap: capecitabine; C225: cetuximab; CPT: camptothecin; BEV: bevacizumab; DXL: docetaxel; CBP: carboplatin; PAN: panitumumab;

PC patients (sample size ranging from 11 to 660 ) in 61 non-controlled studies are listed by Table 6-10.

\section{HIPEC characteristics}

Major technical features of HIPEC procedures in each institution are summarized in Table 11-19. HIPEC was performed using only open technique in 22 institutions and only closed techniques 10 institutions, with 41 institutions used both open and closed techniques. The commonly used chemotherapy agents were mitomycin $\mathrm{C}$ (MMC) alone $\left(n=63\right.$, dosage of $30-50 \mathrm{mg} / \mathrm{m}^{2}$ in $88 \%$ of institutions, median temperature $41.5^{\circ} \mathrm{C}$, ranging from 40 $-43^{\circ} \mathrm{C}$, and median duration $90 \mathrm{~min}$, ranging from 60 - 90 min), oxaliplatin (L-OHP) alone ( $n=43$, dosage of 460 $\mathrm{mg} / \mathrm{m}^{2}$ in $60 \%$ of institutions, median temperature $43^{\circ} \mathrm{C}$, ranging from $40-43^{\circ} \mathrm{C}$; and median duration $60 \mathrm{~min}$ ), and a combination of MMC and cisplatin (CDDP) $(n=$ 24 , dosage of $30-50 \mathrm{mg} / \mathrm{m}^{2}+50-100 \mathrm{mg} / \mathrm{m}^{2}$ in $33 \%$ of institutions).

\section{Primary results for meta-analysis}

\section{Meta-analysis outcomes}

The summarized HRs for OS in the 15 controlled researches was $2.67\left(95 \% \mathrm{CI}, 2.21-3.23, I^{2}=0 \%, P<\right.$ 0.00001) (Figure 2a), suggesting that CRC PC patients could obtain more benefits from CRS plus HIPEC than traditional therapy, without apparent heterogeneity among the studies $\left(P=0.81, I^{2}=0 \%\right)$.

Sensitivity analysis of summarized HR and 95\% CI showed no difference after choosing random effects model and fixed effects model. In terms of sample size difference, 15 researches were divided into three subgroups (sample size $<50,50-100,>100$ ) by a sensitivity study for a stratified meta-analysis. The summarized HR and 95\% CI showed no difference, with no betweensubgroup heterogeneity $\left(P=0.48, I^{2}=0 \%\right.$ ) (Figure $2 b)$. In a sensitivity analysis, four studies with potential heterogeneity was removed due to small sample size [34] 
Table 4: Major Characteristics of Fifteen Controlled Researches on Peritoneal Carcinomatosis (PC) from Colorectal Cancer (CRC) Treated with Cytoreductive Surgery (CRS) plus Hyperthermic Intraperitoneal Chemotherapy (HIPEC) versus Surgery alone with Systemic Chemotherapy (SC) and/or Early Postoperative Intraperitoneal Chemotherapy (EPIC).

\begin{tabular}{|c|c|c|c|c|c|c|c|}
\hline \multirow[b]{2}{*}{ Author/Year/ Country } & \multirow[b]{2}{*}{$\begin{array}{l}\text { Participating } \\
\text { Institutions }\end{array}$} & \multirow[b]{2}{*}{$\begin{array}{l}\text { Study } \\
\text { Period }\end{array}$} & \multirow[b]{2}{*}{ Design } & \multirow[b]{2}{*}{$\begin{array}{l}\text { Level of } \\
\text { Evidence }\end{array}$} & \multirow[b]{2}{*}{$\begin{array}{l}\text { Number } \\
\text { CRC PC }\end{array}$} & \multicolumn{2}{|l|}{ Treatment strategy } \\
\hline & & & & & & HIPEC group & \begin{tabular}{|l} 
Control \\
group
\end{tabular} \\
\hline $\begin{array}{l}\text { Gervais MK/ 2013/ } \\
\text { Canada [38] }\end{array}$ & 1 & 2004-2011 & retrospective & III & $40(40 / 40)$ & $\begin{array}{l}\text { Neoadjuvant SC with/without } \\
\text { neoadjuvant radiotherapy+ } \\
\text { CRS+HIPEC+SC } 25 \text { pts } \\
\text { Neoadjuvant SC: BEV } \\
\text { HIPEC: L-OHP }\left(460 \mathrm{mg} / \mathrm{m}^{2}\right) \text { for } \\
30 \text { min between } 42-44^{\circ} \mathrm{C} \text { using } \\
\text { the Coliseum technique; Before } \\
\text { starting HIEPC, } 5 \text {-FU }\left(400 \mathrm{mg} / \mathrm{m}^{2}\right) \\
\text { and LV }\left(20 \mathrm{mg} / \mathrm{m}^{2}\right) \text { by intravenous } \\
\text { perfusion. } \\
\text { No EPIC } \\
\text { SC: } 5 \text {-FU, LV, L-OHP, and/or CPT- } \\
\text { 11, with or without BEV }\end{array}$ & $\begin{array}{l}\text { Neoadjuvant } \\
\text { SC with/ } \\
\text { without } \\
\text { neoadjuvant } \\
\text { radiotherapy } \\
+ \text { surgery + } \\
\text { SC 15 pts } \\
\text { Neoadjuvant } \\
\text { SC: BEV } \\
\text { SC: 5-FU, } \\
\text { LV, L-OHP, } \\
\text { and/or CPT- } \\
\text { 11, with or } \\
\text { without BEV } \\
\text { No EPIC } \\
\text { No HIPEC }\end{array}$ \\
\hline $\begin{array}{l}\text { Glehen O/ 2004/ France } \\
\text { [13] }\end{array}$ & $\mid \begin{array}{ll}28 & \\
\text { (a } & \text { central } \\
\text { database })\end{array}$ & $1987-2002$ & retrospective & II & $506(506 / 506)$ & 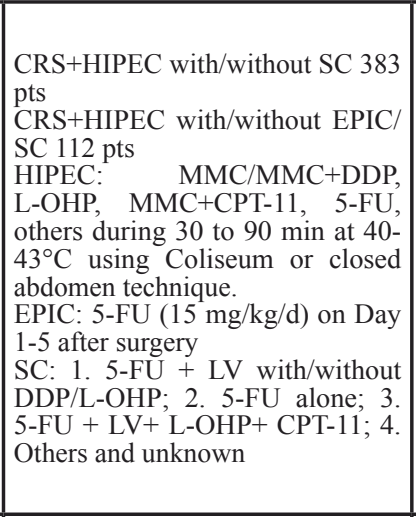 & $\begin{array}{l}\text { CRS+EPIC } \\
\text { with/without } \\
\text { SC 235 pts } \\
\text { EPIC: } \\
\text { 5-FU (15 } \\
\text { mg/kg/d) on } \\
\text { Day 1-5 after } \\
\text { surgery } \\
\text { SC: 1. 5-FU } \\
+ \text { LV with/ } \\
\text { without DDP/ } \\
\text { L-OHP; 2. 2. } \\
\text { 5-FU alone; } 3 . \\
\text { 5-FU + LV+ } \\
\text { L-OHP+ CPT- } \\
\text { 11; 4. Others } \\
\text { and unknown } \\
\text { No HIPEC } \\
\end{array}$ \\
\hline $\begin{array}{l}\text { Goéré D/ 2015/ France } \\
\text { [99] }\end{array}$ & 1 & $2000-2010$ & retrospective & III & $139(139 / 180)$ & $\begin{array}{l}\text { Neoadjuvant } \\
\text { +CRS+HIPEC+SC with/without } \\
\text { EPIC 139 pts } \\
\text { HIPEC: L-OHP+CPT-11 (72\%), } \\
\text { CPT-11 alone (15\%), other items } \\
\text { not reported. } \\
\text { SC: } 1.5 \text {-FU + L-OHP; } 2.5 \text {-FU + } \\
\text { CPT-11; 3. 5-FU alone } \\
\text { EPIC: MMC/5-FU }\end{array}$ & $\begin{array}{l}\text { Neoadjuvant } \\
\text { SC } \\
+ \text { Surgery+SC } \\
41 \text { pts } \\
\text { SC: 1. 5-FU } \\
+ \text { L-OHP; 2. } \\
5-F U+\text { CPT- } \\
\text { 11; 3. 5-FU } \\
\text { alone }\end{array}$ \\
\hline
\end{tabular}

Note: CRC: colorectal cancer; PC: peritoneal carcinomatosis; Pts: patients; MMC: mitomycin C; DDP: cisplatin; FU: fluorouracil; LV: leucovorin; L-OHP: oxaliplatin; CPT-11: irinotecan; Cap: capecitabine; C225: cetuximab; CPT: camptothecin; BEV: bevacizumab; DXL: docetaxel; CBP: carboplatin; PAN: panitumumab;

or asymmetrical sample size between two groups $[14,26$, $40,99]$, but the summary HR was $2.81(95 \% \mathrm{CI}, 2.28-3.48$, $\left.I^{2}=0 \%, P_{\text {heterogeneity }}=0.56\right)$.

There was no statistically significant heterogeneity of HRs for published-time pertinence $(P=0.52)$ (Figure 2c) and geographic-distribution pertinence $(P=0.43)$ (Figure 2d).

\section{Analysis of chemotherapy regimens}

Regarding the effect of different chemotherapy regimens in HIPEC procedure on the efficacy on OS or DFS, 15 researches were divided into 3 subgroups: group of MMC based chemotherapy, group of L-OHP based chemotherapy, and group of other regimens. The heterogeneity showed no significant difference $(P$
$=0.27, I^{2}=24.1 \%$ ), which revealed that difference of chemotherapy regimens of HIPEC was not associated with OS and DFS after CRS and HIPEC in this meta-analysis (Figure 3a). A further analysis of difference in median year survival rate between group of CRS plus HIPEC and group of traditional treatment was conducted by independentsamples $T$ test stratified by MMC and L-OHP subgroups (Figure $3 b$ and Figure 3c).

\section{MMC-based HIPEC procedure}

OS data by MMC-based HIPEC procedure were available in 7 studies with 614 patients $[12,13,15,34,35$, $37,39]$. Due to more patients received MMC regimen in studies by Elias et al. [35] (21 patients for MMC regimen, while 6 patients for L-OHP regimen) and Glehen et al. 
Table 5: Major Characteristics of Fifteen Controlled Researches on Peritoneal Carcinomatosis (PC) from Colorectal Cancer (CRC) Treated with Cytoreductive Surgery (CRS) plus Hyperthermic Intraperitoneal Chemotherapy (HIPEC) versus Surgery alone with Systemic Chemotherapy (SC) and/or Early Postoperative Intraperitoneal Chemotherapy (EPIC).

\begin{tabular}{|c|c|c|c|c|c|c|c|}
\hline \multirow{2}{*}{ Author/Year/ Country } & \multirow{2}{*}{$\begin{array}{l}\text { Participating } \\
\text { Institutions }\end{array}$} & \multirow{2}{*}{ Study Period } & \multirow{2}{*}{ Design } & \multirow{2}{*}{$\begin{array}{l}\text { Level of } \\
\text { Evidence }\end{array}$} & \multirow{2}{*}{$\begin{array}{l}\text { Number of } \\
\text { CRC PC }\end{array}$} & \multicolumn{2}{|l|}{\begin{tabular}{|l} 
Treatment strategy \\
\end{tabular}} \\
\hline & & & & & & HIPEC group & Control group \\
\hline $\begin{array}{l}\text { Huang CQ/ 2014/China } \\
{[39]}\end{array}$ & 1 & 2004-2013 & retrospective & IIa & $62(62 / 62)$ & $\begin{array}{l}\text { CRS+HIPEC+SC with/without PIC } 33 \text { pts } \\
\text { HIPCE: MMC (30 mg) + DDP (120 } \\
\text { mg) for } 90 \mathrm{~min} \text { at } 43.0 \pm 0.5^{\circ} \mathrm{C} \text { using the } \\
\text { Coliseum technique } \\
\text { EPIC: DXL ( } 75 \mathrm{mg} / \mathrm{m}^{2} \text {, on day 1, every } 3 \\
\text { weeks) and CBP (at Calvert formula: area } \\
\text { under the curve, AUC } 5 \text {; on day 1, every } \\
3 \text { weeks) } \\
\text { SC: FOLFOX or FOLFIRI }\end{array}$ & $\begin{array}{l}\text { CRS+ SC with/without PIC } 29 \text { pts } \\
\text { SC: } 0 \text { FOLOX or FOLFIRI } \\
\text { EPIC: DXL (75 } \mathrm{mg} / \mathrm{m}^{2} \text {, on day 1, every } 3 \\
\text { weeks) and CBP (at Calvert formula: AUC } \\
\text { 5; on day 1, every } 3 \text { weeks) } \\
\text { No HIPEC }\end{array}$ \\
\hline $\begin{array}{l}\text { Passot G/2014/ France } \\
{[40]}\end{array}$ & 1 & 2005-2012 & retrospective & IIa & $82(82 / 115)$ & \begin{tabular}{|l|} 
Neoadjuvant SC+CRS+HIPEC 82 pts \\
Neodjuvant SC: 1 . FOLFIRI with/fithout \\
BEV or C225; 2 . FOLFOX with/without \\
BEV or C225;3. Others regimens. \\
HIPEC: L-OHP (360 mg/m²) for 30 min \\
using the closed abdomen technique, not \\
reported the perfusion temperature. \\
No EPIC \\
No SC
\end{tabular} & $\begin{array}{l}\text { Neoadjuvant SC + Surgery + SC } 33 \text { pts } \\
\text { Neadjuvant SC: 1. FOLFIRI with/without } \\
\text { BEV or C225; } 2 \text {. FOLFOX with//thout } \\
\text { BEV or C225; 3. Others regimens. } \\
\text { No EPIC } \\
\text { SC (uncertainty) }\end{array}$ \\
\hline $\begin{array}{l}\text { Verwaal VJ/ } 2003 \\
\text { Netherlands [12] }\end{array}$ & 1 & 1998-2001 & prospective & $\mathrm{Ib}$ & $87(87 / 105)$ & $\begin{array}{l}\text { CRS+HIPEC with/ without SC } 54 \mathrm{pts} \\
\text { HIPEC: MMC }\left(17.5 \mathrm{mg} / \mathrm{m}^{2}\right) \text { for } 90 \mathrm{~min} \\
\text { between } 42-44^{\circ} \mathrm{C} \text { using the Coliseum } \\
\text { technique } \\
\text { No EPIC } \\
\text { SC: } 1.5 \text {-FU }\left(400 \mathrm{mg} / \mathrm{m}^{2}\right)+\mathrm{LV}\left(80 \mathrm{mg} / \mathrm{m}^{2}\right) ; \\
\text { 2. FU +CPT-11 }\left(350 \mathrm{mg} / \mathrm{m}^{2}\right)\end{array}$ & $\begin{array}{l}\text { Surgery and/or SC } 51 \mathrm{pts} \\
\text { SC.1. } 5 \text {-FU }\left(400 \mathrm{mg} / \mathrm{m}^{2}\right)+\mathrm{LV}\left(80 \mathrm{mg} / \mathrm{m}^{2}\right) \text {; } \\
\text { 2. FU + CPT-11 }\left(350 \mathrm{mg} / \mathrm{m}^{2}\right) \\
\text { No EPIC } \\
\text { No HIPEC }\end{array}$ \\
\hline
\end{tabular}

Note: CRC: colorectal cancer; PC: peritoneal carcinomatosis; Pts: patients; MMC: mitomycin C; DDP: cisplatin; FU: fluorouracil; LV: leucovorin; L-OHP: oxaliplatin; CPT-11: irinotecan; Cap: capecitabine; C225: cetuximab; CPT: camptothecin; BEV: bevacizumab; DXL: docetaxel; CBP: carboplatin; PAN: panitumumab;

Table 6: Major Characteristics of Sixty-one Single Arm Researches on Peritoneal Carcinomatosis (PC) from Colorectal Cancer (CRC) Treated with Cytoreductive Surgery (CRS) plus Hyperthermic Intraperitoneal Chemotherapy (HIPEC)

\begin{tabular}{|c|c|c|c|c|c|c|}
\hline Author/ Years/ Country & $\begin{array}{l}\text { Participating } \\
\text { Institutions }\end{array}$ & $\begin{array}{l}\text { Study } \\
\text { Period }\end{array}$ & Design & $\begin{array}{l}\text { Level of } \\
\text { evidence }\end{array}$ & $\begin{array}{l}\text { Number of } \\
\text { CRC PC }\end{array}$ & HIPEC \\
\hline $\begin{array}{l}\text { Alzahrani/ } \quad 2015 / \\
\text { Australia [41] }\end{array}$ & 1 & $\begin{array}{l}1996- \\
2014\end{array}$ & retrospective & III & $\begin{array}{l}205 \\
(205 / 675)\end{array}$ & $\begin{array}{l}\text { Before HIPEC, 5-FU }\left(400 \mathrm{mg} / \mathrm{m}^{2}\right) \text { were delivered by } \\
\text { systemic i.v., L-OHP }\left(350 \mathrm{mg} / \mathrm{m}^{2}\right) \text { for } 30 \mathrm{~min} \text { at } 43^{\circ} \mathrm{C} \text { using } \\
\text { coliseum technique. }\end{array}$ \\
\hline $\begin{array}{l}\text { Beaujard/ 2000/ France } \\
{[42]}\end{array}$ & 1 & $\begin{array}{l}1991- \\
1997\end{array}$ & prospective & $\mathrm{IIb}$ & $27(27 / 86)$ & $\begin{array}{l}\text { MMC }(10 \mathrm{mg} / \mathrm{L}) \text { for } 90 \mathrm{~min} \text { at inflow temperature } 46-49^{\circ} \mathrm{C} \\
\text { using the closed abdomen technique. }\end{array}$ \\
\hline $\begin{array}{l}\text { Bijelic/2008/ Australia } \\
{[[43]}\end{array}$ & 1 & $\begin{array}{l}1981- \\
2004\end{array}$ & retrospective & III & $70(70 / 472)$ & $\begin{array}{l}\text { MMC }\left(10 \mathrm{mg} / \mathrm{m}^{2} \text { for females and } 12.5 \mathrm{mg} / \mathrm{m}^{2} \text { for males) for }\right. \\
90 \mathrm{~min} \text { at about } 42{ }^{\circ} \mathrm{C} \text { using the coliseum technique. }\end{array}$ \\
\hline $\begin{array}{l}\text { Braam/ 2014/ Australia } \\
\text { [44] }\end{array}$ & 2 & $\begin{array}{l}2005- \\
2013\end{array}$ & retrospective & III & $\begin{array}{l}132 \\
(132 / 132)\end{array}$ & $\begin{array}{l}\text { MMC }\left(17.5 \mathrm{mg} / \mathrm{m}^{2} \text { an additional } 8.8 \mathrm{mg} / \mathrm{m}^{2} \text { at an interval }\right. \\
\text { of } 30 \text { and } 60 \mathrm{~min}) \text { for } 90 \mathrm{~min} \text { at } 42^{\circ} \mathrm{C} \text { using the coliseum } \\
\text { technique. }\end{array}$ \\
\hline $\begin{array}{l}\text { Cao/ 2009/ Australia } \\
{[45]}\end{array}$ & 1 & $\begin{array}{l}1995- \\
2008\end{array}$ & retrospective & III & $52(52 / 467)$ & $\begin{array}{l}\text { MMC }\left(10-12.5 \mathrm{mg} / \mathrm{m}^{2}\right) \text { for } 90 \mathrm{~min} \text { at } 42{ }^{\circ} \mathrm{C} \text { using coliseum } \\
\text { technique. }\end{array}$ \\
\hline $\begin{array}{l}\text { Cavaliere/ 2006/ Italy } \\
{[46]}\end{array}$ & 6 & $\begin{array}{l}1996- \\
2005\end{array}$ & retrospective & III & $\begin{array}{l}120 \\
(120 / 120)\end{array}$ & $\begin{array}{l}\text { MMC }\left(3.3 \mathrm{mg} / \mathrm{m}^{2} / \mathrm{L}\right)+\text { DDP }\left(25 \mathrm{mg} / \mathrm{m}^{2} / \mathrm{L}\right) \text { for } 60-90 \mathrm{~min} \text { at } \\
41.5-43^{\circ} \mathrm{C} \text { using the coliseum or closed abdomen technique. } \\
\text { After intravenous administration of } 5 \text {-FU }\left(400 \mathrm{mg} / \mathrm{m}^{2}\right) \text { and } \\
\mathrm{LV}\left(20 \mathrm{mg} / \mathrm{m}^{2}\right) \text {, L-OHP }\left(460 \mathrm{mg} / \mathrm{m}^{2}\right) \text { for } 30 \mathrm{~min} \text { at } 43{ }^{\circ} \mathrm{C} \\
\text { using the coliseum or closed abdomen technique. }\end{array}$ \\
\hline $\begin{array}{l}\text { Ceelen/ 2014/ Belgium } \\
{[47]}\end{array}$ & 1 & $\begin{array}{l}2002- \\
2012\end{array}$ & retrospective & III & $\begin{array}{l}152 \\
(152 / 166)\end{array}$ & $\begin{array}{l}\text { Before HIPEC, LV }\left(20 \mathrm{mg} / \mathrm{m}^{2}\right) \text { and } 5-\mathrm{FU}\left(400 \mathrm{mg} / \mathrm{m}^{2}\right) \text { were } \\
\text { delivered by systemic i.v. L-OHP }\left(460 \mathrm{mg} / \mathrm{m}^{2}\right) \text { or MMC }(35 \\
\left.\mathrm{mg} / \mathrm{m}^{2}\right) \text { for } 30-90 \text { min at } 41{ }^{\circ} \mathrm{C} \text { using coliseum technique. }\end{array}$ \\
\hline $\begin{array}{l}\text { Desantis/ 2014/ France } \\
\text { [48] }\end{array}$ & 1 & $\begin{array}{l}1999- \\
2011\end{array}$ & retrospective & III & $74(74 / 356)$ & $\begin{array}{l}\text { MMC }\left(10 \mathrm{mg} / \mathrm{m}^{2} \text { for females and } 12.5 \mathrm{mg} / \mathrm{m}^{2} \text { for males }\right) \\
\text { for } 90 \mathrm{~min} \text { at } 43^{\circ} \mathrm{C} \text { using coliseum or closed abdomen } \\
\text { technique. }\end{array}$ \\
\hline Elias/ 2004/ France [49] & 1 & $\begin{array}{l}1998- \\
2001\end{array}$ & prospective & $\mathrm{IIb}$ & $24(24 / 24)$ & $\begin{array}{l}\text { One hour before HIPEC, } \mathrm{LV}\left(20 \mathrm{mg} / \mathrm{m}^{2}\right) \text { and } 5 \text {-FU }(400 \mathrm{mg} / \\
\mathrm{m} 2) \text { were delivered by systemic i.v. } \\
\text { HIPEC: } \mathrm{L}-\mathrm{OHP}\left(460 \mathrm{mg} / \mathrm{m}^{2}\right) \text { for } 30 \mathrm{~min} \text { at } 43{ }^{\circ} \mathrm{C} \text { using the } \\
\text { coliseum technique. }\end{array}$ \\
\hline Elias/ 2014/ France [50] & 1 & $\begin{array}{l}1995- \\
2010\end{array}$ & retrospective & III & $\begin{array}{l}119 \\
(119 / 443)\end{array}$ & $\begin{array}{l}\text { MMC }(5,8 \text {, or } 10 \mathrm{mg} / \mathrm{L}) \text { for } 1 \mathrm{~h} \text { between } 41{ }^{\circ} \mathrm{C} \text { and } 44{ }^{\circ} \mathrm{C} \\
\text { using the coliseum technique. } \\
\text { MMC }\left(20 \mathrm{mg} / \mathrm{m}^{2}\right)+\text { DDP }\left(200 \mathrm{mg} / \mathrm{m}^{2}\right) \text { for } 1 \mathrm{~h} \text { between } 41 \\
{ }^{\circ} \mathrm{C} \text { and } 44{ }^{\circ} \mathrm{C} \text { using the coliseum technique. } \\
\mathrm{L}-\mathrm{OHP} 460 \mathrm{mg} / \mathrm{m}^{2} \text { over } 30 \mathrm{~min} \text { at } 43^{\circ} \mathrm{C} \text { using the coliseum } \\
\text { technique. } \\
\text { MMC }\left(12.9+/-3.8 \mathrm{mg} / \mathrm{m}^{2}\right) \text { for } 90 \text { min between } 41^{\circ} \mathrm{C} \text { and } 42 \\
{ }^{\circ} \mathrm{C} \text { using closed abdomen technique. }\end{array}$ \\
\hline
\end{tabular}

Note: MMC: mitomycin C; DDP: cisplatin; FU: fluorouracil; LV: leucovorin; L-OHP: oxaliplatin; CPT-11: irinotecan; NR: not reported 
[13] (322 patients for MMC regimen, while 32 patients for L-OHP regimen and 29 patients for others), these two studies were included in MMC subgroup. The stratification analysis showed that OS of patients receiving HIPEC by MMC was significantly improved $(\mathrm{HR}=2.88,95 \% \mathrm{CI}$, 2.26-3.68, $I^{2}=0 \%, P<0.00001$ ) (Figure $3 \mathrm{a}$ ), with $1-$, 3 -, and 5-year survival rates of $79.5 \%, 38.8 \%$, and $34 \%$, respectively (Figure $3 \mathrm{~b}$ ). In comparison, the corresponding survival rates in the traditional group were $54.9 \%, 18.3 \%$, and $9.7 \%$, respectively (Figure $3 \mathrm{~b}$ ).

\section{L-OHP-based chemotherapy in HIPEC procedure}

Four studies using L-OHP based chemotherapy in HIPEC procedures of 283 patients [28, 36, 38, 40, 99]. A statistically significant benefit for OS was revealed in HIPEC group $\left(\mathrm{HR}=2.18,95 \% \mathrm{CI}, 1.57-3.04, I^{2}=0 \%, P\right.$ $<0.00001$ ) (Figure 3a), with the 1-, 3-, and 5-year survival rates of $93 \%, 59 \%$, and $43 \%$, respectively in HIPEC group vs. $63 \%, 25 \%$, and $14 \%$, respectively in traditional group (Figure 3c).

\section{Other chemotherapy regimes in HIPEC procedure}

Three trials $[14,26,29]$ were identified as the subgroup of other regimen due to difficulties in identifying them as MMC subgroup or L-OHP subgroup since mixed chemotherapy regimens were used in HIPEC during the whole disease course. A significant survival benefit in HIPEC group vs. traditional group ( $\mathrm{HR}=3.90,95 \%$ CI, 1.73-8.81, $\left.I^{2}=0 \%, P<0.00001\right)$ was demonstrated (Figure 3a).

\section{Publication bias}

Publication bias was evaluated with funnel plot analyses, as shown in Figure 4, and the funnel plot was symmetric. No apparent publication bias was found in our OS meta-analysis with Begg's test $\left(\mathrm{z}_{\text {continuity corrected }}=0.99\right.$, $\operatorname{Pr}>|\mathrm{z}|_{\text {continuity corrected }}=0.32$ ) (Figure $\left.4 \mathrm{a}\right)$, or with Egger's test $(\mathrm{t}=0.82, P>|\mathrm{t}|=0.427,95 \% \mathrm{CI}$ of bias: $-0.49 \sim 1.1)$ (Figure $4 b$ ). a

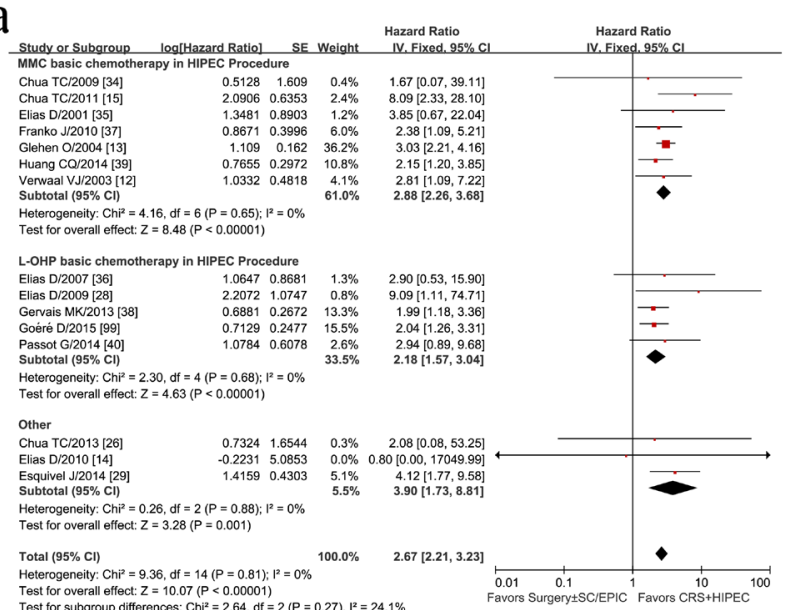

C

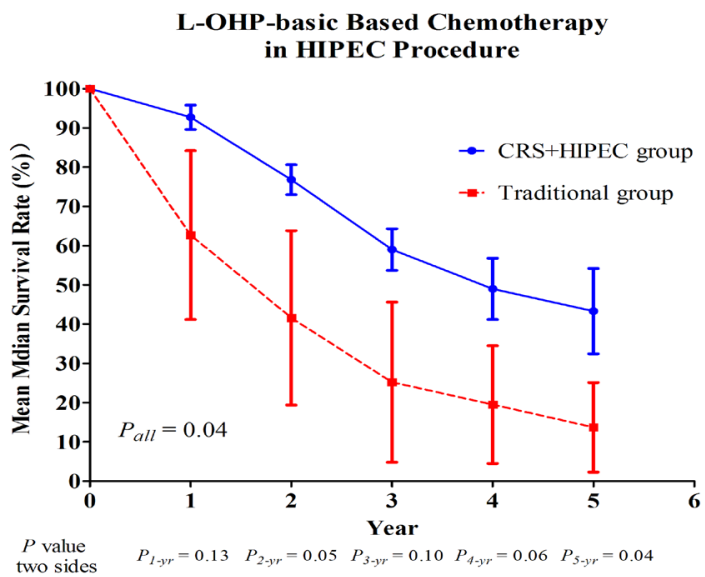

$\mathrm{b}$

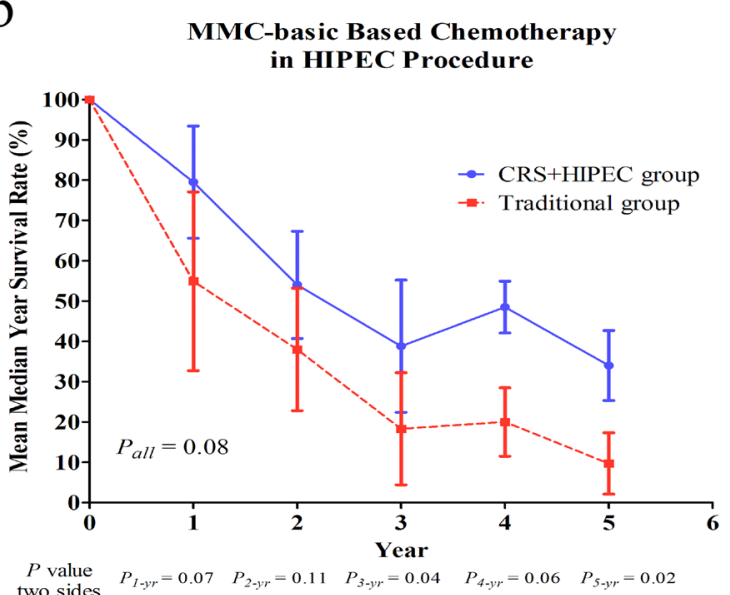

$\mathrm{d}$

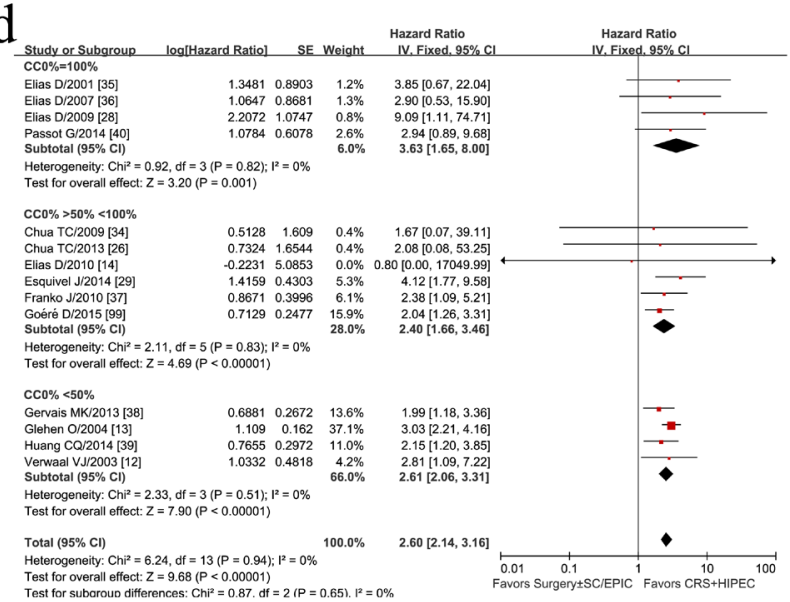

Figure 3: Forest plots of 15 studies evaluating heterogeneity test of chemotherapy regimens difference (MMC based chemotherapy; L-OHP based chemotherapy; others) in HIPEC procedure (a); The difference of mean year survival rate between CRS+HIPEC group and traditional group for MMC-basic (Mitomycin C, MMC) HIPEC procedure (b), for L-OHPbasic (Oxaliplatin, L-OHP) HIPEC procedure (c); Forest plots of 15 studies evaluating heterogeneity test of the proportion of $\mathrm{CC} 0$ difference $(\mathbf{d})$. 
Table 7: Major Characteristics of Sixty-one Single Arm Researches on Peritoneal Carcinomatosis (PC) from Colorectal Cancer (CRC) Treated with Cytoreductive Surgery (CRS) plus Hyperthermic Intraperitoneal Chemotherapy (HIPEC)

\begin{tabular}{|c|c|c|c|c|c|c|}
\hline Author/ Years/ Country & $\begin{array}{l}\text { Participating } \\
\text { Institutions }\end{array}$ & Study Period & Design & $\begin{array}{l}\text { Level of } \\
\text { evidence }\end{array}$ & $\begin{array}{l}\text { Number of CRC } \\
\text { PC }\end{array}$ & HIPEC \\
\hline $\begin{array}{l}\text { Evers/2011/ Netherlands } \\
\text { [51] }\end{array}$ & 1 & 2001-2009 & retrospective & III & $108(108 / 194)$ & $\begin{array}{l}\text { MMC }\left(35 \mathrm{mg} / \mathrm{m}^{2}\right) \text { for } 90 \mathrm{~min} \text { at } 40-41{ }^{\circ} \mathrm{C} \text {, perfusion } \\
\text { mode not reported. }\end{array}$ \\
\hline $\begin{array}{l}\text { Faron M/ 2016/ France } \\
{[100]}\end{array}$ & 1 & 2003-2012 & retrospective & III & $173(173 / 173)$ & $\begin{array}{l}\text { Before HIPEC, LV }\left(20 \mathrm{mg} / \mathrm{m}^{2}\right) \text { and } 5 \text {-FU }(400 \mathrm{mg} \text { / } \\
\mathrm{m} 2) \text { were delivered by systemic i.v. } \\
\text { HIPEC: } \mathrm{L}-\mathrm{OHP}\left(300 \mathrm{mg} / \mathrm{m}^{2}\right) \text { and CPT- } 11(200 \mathrm{mg} / \\
\left.\mathrm{m}^{2}\right) \text { for } 30 \mathrm{~min} \text { between } 43^{\circ} \mathrm{C} \text { using closed abdomen } \\
\text { technique. }\end{array}$ \\
\hline $\begin{array}{l}\text { Franko/ 2008/ America } \\
{[52]}\end{array}$ & 1 & 2001-2007 & retrospective & III & $65(65 / 65)$ & $\begin{array}{l}\text { MMC }\left(40 \mathrm{mg} / \mathrm{m}^{2}\right) \text { for } 90 \mathrm{~min} \text { using closed abdomen } \\
\text { technique. (have not reported the liquid perfusion } \\
\text { temperature) }\end{array}$ \\
\hline $\begin{array}{l}\text { Frøysnes/ } \\
\text { Norway[103] 2016/ }\end{array}$ & 1 & 2004-2013 & retrospective & III & $119(119 / 144)$ & $\begin{array}{l}\mathrm{MMC}\left(35 \mathrm{mg} / \mathrm{m}^{2}\right) \text { for } 90 \mathrm{~min} \text { between } 39.5^{\circ} \mathrm{C} \text { and } \\
41.2^{\circ} \mathrm{C} \text { using closed abdomen technique until } 2008 \text {, } \\
\text { and thereafter a closed technique with open abdomen }\end{array}$ \\
\hline Glehen/ 2003/ France [53] & 1 & $1998-2001$ & prospective & $\mathrm{IIb}$ & $26(26 / 56)$ & $\begin{array}{l}\text { MMC }(0.7 \mathrm{mg} / \mathrm{kg}) \text { for } 90 \mathrm{~min} \text { at } 46-48{ }^{\circ} \mathrm{C} \text { using } \\
\text { closed abdomen technique. }\end{array}$ \\
\hline Glehen/ 2004/ France [16] & 1 & 1989-2002 & retrospective & III & $53(53 / 53)$ & $\begin{array}{l}\text { MMC (total dose } 40-60 \mathrm{mg} \text { ) for } 90 \mathrm{~min} \text { at } 46-48^{\circ} \mathrm{C} \\
\text { using closed abdomen technique. }\end{array}$ \\
\hline Glehen/ 2010/ France [54] & 25 & $1989-2007$ & retrospective & III & $523(523 / 1290)$ & $\begin{array}{l}\text { MMC }\left(30-50 \mathrm{mg} / \mathrm{m}^{2}\right) \text { with or without DDP }(50- \\
\left.100 \mathrm{mg} / \mathrm{m}^{2}\right) \text { for } 60-120 \mathrm{~min} \text { at } 41-42.5^{\circ} \mathrm{C} \text { using the } \\
\text { coliseum or closed abdomen technique. } \\
\mathrm{L}-\mathrm{OHP}\left(360-460 \mathrm{mg} / \mathrm{m}^{2}\right) \text { with or without CPT-11 } \\
\left(100-200 \mathrm{mg} / \mathrm{m}^{2}\right) \text { with or without intravenous } 5 \text {-FU } \\
\text { and LV delivered over } 30 \text { min at } 43^{\circ} \mathrm{C} \text { using the } \\
\text { coliseum or closed abdomen technique. }\end{array}$ \\
\hline $\begin{array}{l}\text { Gomes da Silva/ 2005/ } \\
\text { America [55] }\end{array}$ & 1 & 1981-2004 & retrospective & III & $11(11 / 11)$ & $\begin{array}{l}\text { MMC }\left(10 \mathrm{mg} / \mathrm{m}^{2} \text { in females and } 12.5 \mathrm{mg} / \mathrm{m}^{2} \text { in }\right. \\
\text { males) for } 90 \mathrm{~min} \text { at } 41-42{ }^{\circ} \mathrm{C} \text { using closed abdomen } \\
\text { technique. }\end{array}$ \\
\hline $\begin{array}{l}\text { Gusani/ 2008/ America } \\
{[56]}\end{array}$ & 1 & $2002-2005$ & retrospective & III & $28(25 / 122)$ & $\begin{array}{l}\text { MMC }(30 \mathrm{mg}) \text { for } 60 \mathrm{~min} \text { at } 40-42^{\circ} \mathrm{C} \text { using closed } \\
\text { abdomen technique, after } 60 \mathrm{~min} \text {, additional MMC } \\
(10 \mathrm{mg}) \text { was added for } 40 \text { more min. }\end{array}$ \\
\hline $\begin{array}{l}\text { Hamilton/ 2011/ Canada } \\
\text { [57] }\end{array}$ & 1 & $2000-2008$ & retrospective & III & $31(31 / 101)$ & $\begin{array}{l}\text { MMC (12-15 mg) for } 90 \mathrm{~min} \text { at } 40-42{ }^{\circ} \mathrm{C} \text { using } \\
\text { coliseum technique. }\end{array}$ \\
\hline $\begin{array}{l}\text { Hompes/ 2012/ Belgium } \\
{[58]}\end{array}$ & 6 & 2004-2008 & prospective & $\mathrm{IIb}$ & $39(39 / 48)$ & $\begin{array}{l}\mathrm{L} \text {-OHP }\left(460 \mathrm{mg} / \mathrm{m}^{2}\right) \text { for } 30 \mathrm{~min} \text { at } 41-42{ }^{\circ} \mathrm{C} \text { using } \\
\text { coliseum technique. Before HIPEC, systemic } \\
\mathrm{LV}\left(20 \mathrm{mg} / \mathrm{m}^{2}\right) \text { and 5-FU }\left(400 \mathrm{mg} / \mathrm{m}^{2}\right) \text { were } \\
\text { administered. }\end{array}$ \\
\hline
\end{tabular}

Note: MMC: mitomycin C; DDP: cisplatin; FU: fluorouracil; LV: leucovorin; L-OHP: oxaliplatin; CPT-11: irinotecan; NR: not reported

\section{Summary of HIPEC-related data}

In 15 controlled studies and 59 single-arm studies, HIPEC-related outcomes including survival rates, median OS and 95\% CI, DFS/RFS, PFS, follow-up time, morbidity, and mortality, are summarized in Table 20-25 and Figure 5

$\mathbf{a}$

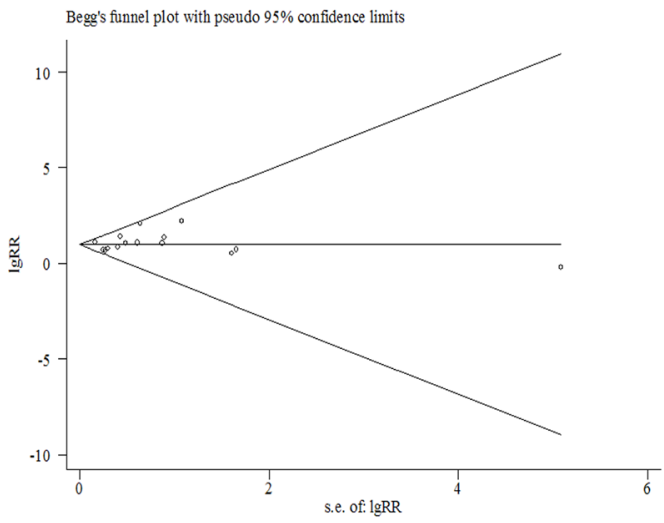

\section{Adverse events}

In controlled studies, the mean $( \pm \mathrm{SD})$ mortality and morbidity rates were $4.3 \%( \pm 3.7 \%)$ and $19.8 \%( \pm$ 9.2\%) in HIPEC groups, 6.2\% $( \pm 4.2 \%)$ and $20.5 \%( \pm$ $12.3 \%$ ) in traditional groups, respectively (Table 20$25)$. No significant difference in mortality $(P=0.423)$ or

b

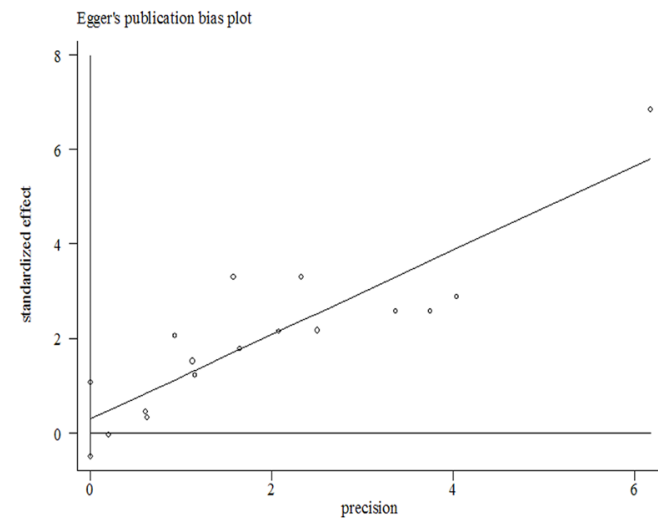

Figure 4: Funnel plots of this meta-analysis by Begg's test (a), and by Egger's test (b). 
Table 8: Major Characteristics of Sixty-one Single Arm Researches on Peritoneal Carcinomatosis (PC) from Colorectal Cancer (CRC) Treated with Cytoreductive Surgery (CRS) plus Hyperthermic Intraperitoneal Chemotherapy (HIPEC)

\begin{tabular}{|c|c|c|c|c|c|c|}
\hline Author/ Years/ Country & $\begin{array}{l}\text { Participating } \\
\text { Institutions }\end{array}$ & $\begin{array}{l}\text { Study } \\
\text { Period }\end{array}$ & Design & $\begin{array}{l}\text { Level of } \\
\text { evidence }\end{array}$ & $\begin{array}{l}\text { Number } \\
\text { CRC PC }\end{array}$ & HIPEC \\
\hline Hompes/ 2014/ Belgium [59] & 2 & $\begin{array}{l}2004-2006 \\
2006-2010\end{array}$ & retrospective & IIb & $95(95 / 95)$ & $\begin{array}{l}\text { MMC }\left(35 \mathrm{mg} / \mathrm{m}^{2}\right) \text { for } 90 \mathrm{~min} \text { at } 41-42{ }^{\circ} \mathrm{C} \text { using coliseum or } \\
\text { closed abdomen technique. } \\
\text { Before HIPEC, LV }\left(20 \mathrm{mg} / \mathrm{m}^{2}\right) \text { and } 5 \text {-FU }\left(400 \mathrm{mg} / \mathrm{m}^{2}\right) \text { were } \\
\text { delivered by systemic i.v. L-OHP }\left(460 \mathrm{mg} / \mathrm{m}^{2}\right) \text { for } 30 \mathrm{~min} \\
\text { at } 41-42{ }^{\circ} \mathrm{C} \text { using coliseum or closed abdomen technique. }\end{array}$ \\
\hline Iversen/2013/ Denmark [60] & 1 & 2006-2012 & retrospective & III & $34(34 / 80)$ & $\begin{array}{l}\text { MMC }\left(35 \mathrm{mg} / \mathrm{m}^{2}\right) \text { for } 90 \mathrm{~min} \text { at } 41.0-42.5^{\circ} \mathrm{C} \text { using coliseum } \\
\text { technique. }\end{array}$ \\
\hline $\begin{array}{l}\text { Kecmanovic/ 2005/ Serbia } \\
\text { and Montenegro [61] }\end{array}$ & 1 & $1996-2003$ & retrospective & III & $18(18 / 18)$ & $\begin{array}{l}\text { MMC }\left(12.5 \mathrm{mg} / \mathrm{m}^{2}, \max .25 \mathrm{mg} \text { for males; } 10.0 \mathrm{mg} / \mathrm{m}^{2} \text {, }\right. \\
\text { max. } 20 \mathrm{mg} \text { for females) for } 120 \mathrm{~min} \text { at } 42^{\circ} \mathrm{C} \text { using closed } \\
\text { abdomen technique }\end{array}$ \\
\hline $\begin{array}{l}\text { Kianmanesh/2007/ France } \\
{[62]}\end{array}$ & 1 & $1992-2005$ & retrospective & III & $43(43 / 43)$ & $\begin{array}{l}\mathrm{MMC}(120 \mathrm{mg})+\mathrm{DDP}\left(200 \mathrm{mg} / \mathrm{m}^{2}\right) \text { for } 90-120 \mathrm{~min} \text { at } 47-50 \\
{ }^{\circ} \mathrm{C} \text { using coliseum or closed abdomen technique. }\end{array}$ \\
\hline \begin{tabular}{|l|} 
Klaver/ 2011/ Netherlands \\
{$[63]$}
\end{tabular} & 1 & $1997-2008$ & retrospective & III & $21(21 / 21)$ & $\begin{array}{l}\text { MMC }\left(35 \mathrm{mg} / \mathrm{m}^{2}\right) \text { for } 90 \mathrm{~min} \text { at } 41^{\circ} \mathrm{C} \text { using coliseum } \\
\text { technique. }\end{array}$ \\
\hline $\begin{array}{l}\text { Klaver/ 2012/ Netherlands } \\
{[64]}\end{array}$ & 2 & 1996-2010 & retrospective & III & $17(17 / 24)$ & $\begin{array}{l}\text { MMC or L-OHP for } 90 \text { min at } 42^{\circ} \mathrm{C} \text { using coliseum } \\
\text { technique. }\end{array}$ \\
\hline $\begin{array}{l}\text { Kuijpers/ 2013/ Netherlands } \\
{[65]}\end{array}$ & 6 & $1995-2012$ & retrospective & III & $660(660 / 960)$ & $\begin{array}{l}\text { MMC }\left(35 \mathrm{mg} / \mathrm{m}^{2}\right) \text { (in three fractions (one half, one fourth, } \\
\text { and one fourth of the total dose)) for } 90 \mathrm{~min} \text { at } 41-42{ }^{\circ} \mathrm{C} \\
\text { using coliseum technique. }\end{array}$ \\
\hline $\begin{array}{l}\text { Kuijpers/ 2014/ Netherlands } \\
{[66]}\end{array}$ & 1 & 2004-2012 & retrospective & III & $73(73 / 73)$ & $\begin{array}{l}\text { MMC }\left(35 \mathrm{mg} / \mathrm{m}^{2}\right) \text { for } 90 \mathrm{~min} \text { at } 41-42{ }^{\circ} \mathrm{C} \text { using coliseum } \\
\text { technique. }\end{array}$ \\
\hline Lanuke/ 2009/ Canada [67] & 1 & $2000-2008$ & prospective & IIb & $31(31 / 101)$ & $\begin{array}{l}\text { MMC }(12-15 \mathrm{mg}) \text { for } 60 \mathrm{~min} \text { at } 40-42{ }^{\circ} \mathrm{C} \text { using coliseum } \\
\text { technique. }\end{array}$ \\
\hline Levine/ 2014/ America [68] & 1 & $1991-2013$ & retrospective & III & $232(232 / 1000)$ & $\begin{array}{l}\text { MMC (30 mg) for } 60-90 \mathrm{~min} \text { at } 38.5-43{ }^{\circ} \mathrm{C} \text { using coliseum } \\
\text { technique; L-OHP }\left(200 \mathrm{mg} / \mathrm{m}^{2}\right) \text { for selected patients. }\end{array}$ \\
\hline $\begin{array}{|lll|}\begin{array}{l}\text { Maillet } \\
{[101]}\end{array} & \text { M/ 2016/ France } \\
\end{array}$ & 4 & 2004-2012 & retrospective & III & $231(231 / 231)$ & NR \\
\hline $\begin{array}{l}\text { McConnell/ 2013/ Canada } \\
{[69]}\end{array}$ & 1 & $2000-2011$ & retrospective & III & $245(245 / 245)$ & $\begin{array}{l}\text { MMC }(12-15 \mathrm{mg}) \text { for } 60 \mathrm{~min} \text { at } 40-42{ }^{\circ} \mathrm{C} \text { using coliseum or } \\
\text { closed abdomen technique. } \\
\mathrm{L}-\mathrm{OHP}\left(400 \mathrm{mg} / \mathrm{m}^{2}\right) \text { for } 60 \mathrm{~min} \text { at } 40-42^{\circ} \mathrm{C} \text { using coliseum } \\
\text { or closed abdomen technique with a simultaneous dose of } \\
\text { intravenous } 5-\mathrm{FU}(800 \mathrm{mg}) . .\end{array}$ \\
\hline Nikolic/2014/ Serbia [70] & 1 & 2005-2012 & retrospective & III & $61(61 / 61)$ & $\begin{array}{l}\mathrm{L}-\mathrm{OHP}\left(410 \mathrm{mg} / \mathrm{m}^{2}\right) \text { for } 30-60 \mathrm{~min} \text { at } 41{ }^{\circ} \mathrm{C} \text { using closed } \\
\text { abdomen technique. }\end{array}$ \\
\hline
\end{tabular}

Note: MMC: mitomycin C; DDP: cisplatin; FU: fluorouracil; LV: leucovorin; L-OHP: oxaliplatin; CPT-11: irinotecan; NR: not reported

morbidity $(P=0.815)$ was detected between HIPEC group and traditional group by $T$ test. In the integrated HIPECrelated data of 76 studies, mean mortality and morbidity was $2.8 \%( \pm 2.9 \%)$ and $33.0( \pm 13.4 \%)$, respectively.

\section{DISCUSSION}

Due to the tumor biologic characteristics of colorectal cancer, about $10-13 \%$ patients have already a

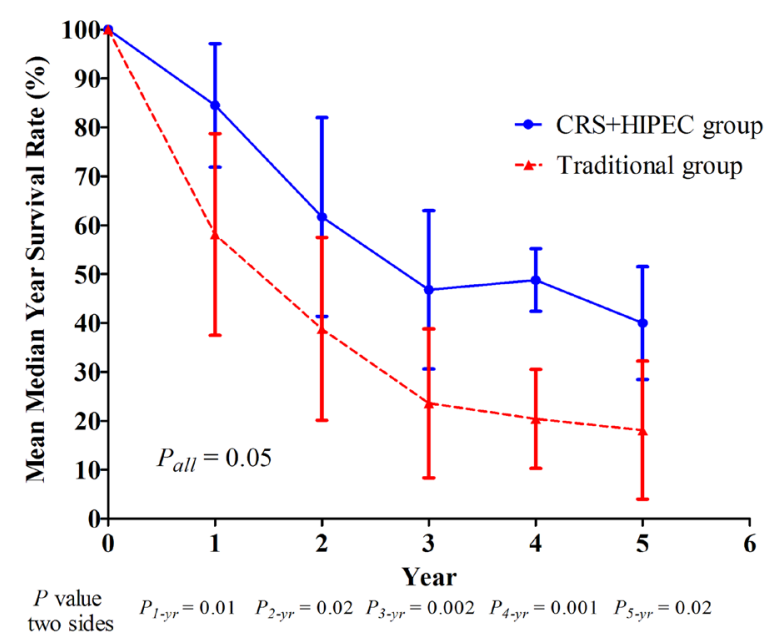

b

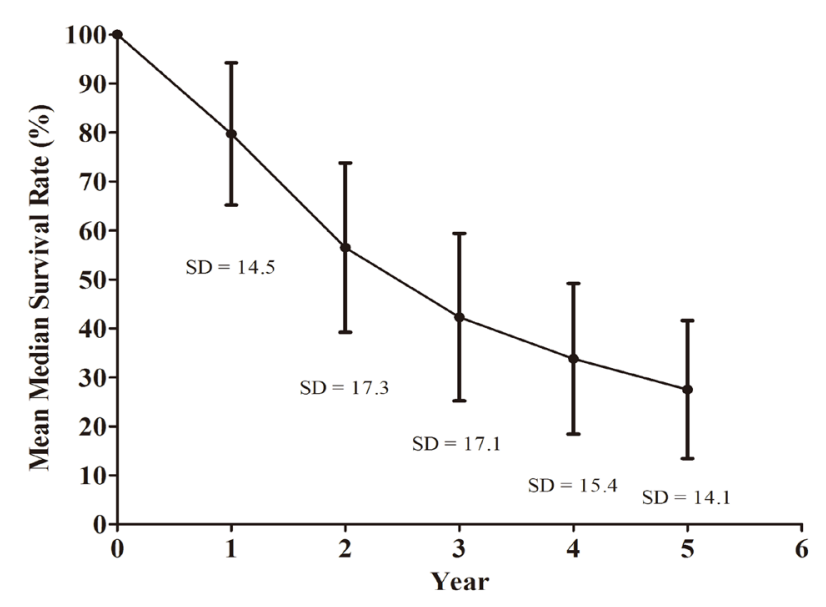

Figure 5: The summarized median year survival rates between CRS+HIPEC group and traditional group for 15 controlled studies (a); The summarized median year survival rates on 76 HIPEC related studies (b). 
Table 9: Major Characteristics of Sixty-one Single Arm Researches on Peritoneal Carcinomatosis (PC) from Colorectal Cancer (CRC) Treated with Cytoreductive Surgery (CRS) plus Hyperthermic Intraperitoneal Chemotherapy (HIPEC)

\begin{tabular}{|c|c|c|c|c|c|c|}
\hline Author/ Years/ Country & $\begin{array}{l}\text { Participating } \\
\text { Institutions }\end{array}$ & $\begin{array}{l}\text { Study } \\
\text { Period }\end{array}$ & Design & $\begin{array}{l}\text { Level of } \\
\text { evidence }\end{array}$ & $\begin{array}{l}\text { Number of } \\
\text { CRC PC }\end{array}$ & HIPEC \\
\hline Passot/ 2012/ France [21] & 1 & 1991-2010 & retrospective & III & $120(120 / 120)$ & $\begin{array}{l}\text { MMC }(10 \mathrm{mg} / \mathrm{ml} \text {, total dose } 40-60 \mathrm{mg}) \text { for } 90 \mathrm{~min} \text { at } 46-48^{\circ} \mathrm{C} \\
\text { using closed abdomen technique. } \\
\text { MMC }(0.7 \mathrm{mg} / \mathrm{kg})+\mathrm{CPT}-11\left(100 \mathrm{mg} / \mathrm{m}^{2}\right) \text { for } 90 \mathrm{~min} \text { at } 44-46 \\
{ }^{\circ} \mathrm{C} \text { using closed abdomen technique. } \\
\mathrm{MMC}\left(30-50 \mathrm{mg} / \mathrm{m}^{2}\right) \text { with or without DDP }\left(50-100 \mathrm{mg} / \mathrm{m}^{2}\right) \\
\text { for } 60-120 \mathrm{~min} \text { at } 41-42.5{ }^{\circ} \mathrm{C} \text { using coliseum technique or } \\
\text { closed abdomen technique. } \\
\mathrm{L}-\mathrm{OHP}\left(360-460 \mathrm{mg} / \mathrm{m}^{2}\right) \text { with or without CPT-11 }(100-200 \\
\left.\mathrm{mg} / \mathrm{m}^{2}\right) \text { with or without intravenous } 5 \text {-FU and LV for } 30 \\
\text { min at } 43^{\circ} \mathrm{C} \text { using coliseum technique or closed abdomen } \\
\text { technique. }\end{array}$ \\
\hline Passot/ 2016/ France [104] & 1 & 1989-2015 & retrospective & III & $342(342 / 1,125)$ & Idem (Passot/ 2012/ France $\left.{ }^{[21]}\right)$ \\
\hline Pilati/ 2003/ Italy [71] & 1 & 1995-2001 & retrospective & III & $46(46 / 46)$ & $\begin{array}{l}\text { MMC }\left(3.3 \mathrm{mg} / \mathrm{m}^{2} / \mathrm{L}\right) \text { with or without } \mathrm{DDP}\left(25 \mathrm{mg} / \mathrm{m}^{2} / \mathrm{L}\right) \text { for } \\
90 \mathrm{~min} \text { at } 41.2-42.1^{\circ} \mathrm{C} \text { using coliseum technique or closed } \\
\text { abdomen technique. }\end{array}$ \\
\hline $\begin{array}{l}\text { Prada-Villaverde/ 2014/ } \\
\text { Spain [72] }\end{array}$ & 15 & $2000-2011$ & retrospective & III & $539(539 / 539)$ & $\begin{array}{l}\text { MMC or L-OHP for } 30-120 \text { min at } 40-43^{\circ} \mathrm{C} \text { using coliseum or } \\
\text { closed abdomen technique. }\end{array}$ \\
\hline Quenet/ 2011/ France [73] & 2 & $\begin{array}{l}1998-2007 \\
2002-2007\end{array}$ & prospective & $\mathrm{IIb}$ & $146(146 / 146)$ & $\begin{array}{l}\text { L-OHP }\left(460 \mathrm{mg} / \mathrm{m}^{2}\right) \text { with intravenous } 5-\mathrm{FU}\left(400 \mathrm{mg} / \mathrm{m}^{2}\right) \\
\text { and LV }\left(20 \mathrm{mg} / \mathrm{m}^{2}\right) \text { for } 30 \text { min at } 42-45^{\circ} \mathrm{C} \text { using coliseum } \\
\text { technique. } \\
\text { L-OHP }\left(300 \mathrm{mg} / \mathrm{m}^{2}\right) \text { with CPT-11 }\left(200 \mathrm{mg} / \mathrm{m}^{2}\right) \text { with } \\
\text { intravenous } 5-\mathrm{FU}\left(400 \mathrm{mg} / \mathrm{m}^{2}\right) \text { and LV }\left(20 \mathrm{mg} / \mathrm{m}^{2}\right) \text { for } 30 \mathrm{~min} \\
\text { at } 42-45^{\circ} \mathrm{C} \text { using coliseum technique. }\end{array}$ \\
\hline Rivard/ 2014/ Canada[74] & 1 & 2003-2011 & retrospective & III & $68(68 / 68)$ & NR \\
\hline Rodt/ 2013/ Denmark [75] & 1 & 2006-2011 & retrospective & III & $19(19 / 35)$ & NR \\
\hline Shen/ 2004/ America [20] & 1 & 1991-2002 & retrospective & III & $77(77 / 77)$ & $\begin{array}{l}\text { MMC (total dose } 30 \mathrm{mg} \text { ) for } 60-120 \mathrm{~min} \text { at } 38.5-43{ }^{\circ} \mathrm{C} \text { using } \\
\text { closed abdomen technique. }\end{array}$ \\
\hline Shen/ 2008/ America [76] & 1 & 1992-2005 & retrospective & III & $55(55 / 150)$ & $\begin{array}{l}\text { MMC (total dose } 30 \mathrm{mg} \text { ) for } 60-120 \mathrm{~min} \text { at } 38.5-43^{\circ} \mathrm{C} \text { using } \\
\text { closed abdomen technique. }\end{array}$ \\
\hline $\begin{array}{lcr}\text { Simkens GA/ 2015/ } \\
\text { Netherlands [102] }\end{array}$ & 1 & $2007-2013$ & retrospective & III & $133(133 / 133)$ & $\begin{array}{l}\text { MMC }\left(35 \mathrm{mg} / \mathrm{m}^{2}\right) \text { for } 90 \mathrm{~min} \text { at } 41.1^{\circ} \mathrm{C} \text { using open-coliseum } \\
\text { technique. }\end{array}$ \\
\hline $\begin{array}{l}\text { Swellengrebel/ } 2009 / \\
\text { Netherlands [77] }\end{array}$ & 1 & 1999-2005 & retrospective & III & $92(92 / 92)$ & $\begin{array}{l}\text { MMC }\left(35 \mathrm{mg} / \mathrm{m}^{2}\right) \text { for } 90 \mathrm{~min} \text { at } 41-42{ }^{\circ} \mathrm{C} \text { using coliseum } \\
\text { technique. }\end{array}$ \\
\hline
\end{tabular}

Note: MMC: mitomycin C; DDP: cisplatin; FU: fluorouracil; LV: leucovorin; L-OHP: oxaliplatin; CPT-11: irinotecan; NR: not reported

progressed to PC when CRC is diagnosed [3, 7], which has a poor prognosis. In order to improve the efficacy, a comprehensive treatment strategy with combination of CRS plus HIPEC had been developed. With wide application of this treatment, CRS plus HIPEC has been proved capable to achieve better survival in selected patients with PC from colorectal cancer.

This meta-analysis of 15 controlled studies demonstrated that CRS+HIPEC comprehensive therapeutic strategy could bring significant survival benefit for selected CRC PC patients than traditional treatment of palliative surgery alone or systemic chemotherapy (HR $=2.67,95 \%$ CI 2.21-3.23, $P<0.00001)$. In addition, the summarizing analysis of these 76 studies showed that the median OS was about 29 months in HIPEC group, which is significant longer compared with median OS of 17.9 months for CRC PC patients receiving contemporary chemotherapy reported by Kerscher et al $(n=2,406)$ [7]. These results provide further supporting evidence that CRS+HIPEC as the principal comprehensive treatment can bring more survival benefit to selected patients with CRC PC than traditional therapy.

The different regimens used in chemotherapy may be one potential confounding factor for survival outcomes. In order to investigate the influence of chemotherapy regimens on postoperative survival, a stratification analysis between MMC based regimens and L-OHP based regimens was conducted. The results of heterogeneity showed no significant difference $(P=0.50)$. These results are inconsistent with the reports by Elias et al [14], which showed that OS advantage for L-OHP regimens over nonL-OHP regimens (32 vs. 25 months, $P=0.02$ ). However, L-OHP used in HIPEC was not an independent prognostic factor for survival in the study of Elias and colleagues. A multi-center retrospective controlled study reported by Prada-Villaverde et al. [72] showed that of 539 patients undergoing CRS plus HIPEC, L-OHP based HIPEC and MMC based HIPEC achieved similar median OS (31.4 vs. 32.7 months, $P=0.925)$. Similarly, the study of Hompes et al. [59] yielded the same conclusion that there was not obvious benefit in OS for HIPEC with L-OHP (37.1 months) or MMC (26.5 months) $(P=0.45)$. Although different chemotherapy regimens in HIPEC may have an effect on stability and reliability of this meta-analysis, the result of heterogeneity analysis was in accordance with above studies. As a result, both MMC and L-OHP were the feasible chemotherapy drugs in HIPEC for CRC PC patients to achieve similar efficacy.

Moreover, there are also some doubts that different chemotherapy in intravenous or postoperative 
Table 10: Major Characteristics of Sixty-one Single Arm Researches on Peritoneal Carcinomatosis (PC) from Colorectal Cancer (CRC) Treated with Cytoreductive Surgery (CRS) plus Hyperthermic Intraperitoneal Chemotherapy (HIPEC)

\begin{tabular}{|c|c|c|c|c|c|c|}
\hline Author/ Years/ Country & $\begin{array}{l}\text { Participating } \\
\text { Institutions }\end{array}$ & Study Period & Design & $\begin{array}{l}\text { Level of } \\
\text { evidence }\end{array}$ & $\begin{array}{l}\text { Number of CRC } \\
\text { PC }\end{array}$ & HIPEC \\
\hline Tabrizian/ 2014/ America [78] & 1 & $2007-2012$ & retrospective & III & $51(51 / 170)$ & $\begin{array}{l}\text { MMC (total dose } 40 \mathrm{mg} \text { ) for } 90 \mathrm{~min} \text { at } 41-43{ }^{\circ} \mathrm{C} \text { using } \\
\text { closed abdomen technique. }\end{array}$ \\
\hline Teo/ 2013/ Singapore [79] & 1 & 2001-2012 & retrospective & III & $28(28 / 100)$ & $\begin{array}{l}\text { MMC for } 60 \mathrm{~min} \text { at } 42{ }^{\circ} \mathrm{C} \text { using closed abdomen } \\
\text { technique. }\end{array}$ \\
\hline Teo/ 2014/ Singapore [80] & 1 & 2001-2012 & retrospective & III & $35(35 / 35)$ & $\begin{array}{l}\text { MMC for } 60 \mathrm{~min} \text { at } 42{ }^{\circ} \mathrm{C} \text { using closed abdomen } \\
\text { technique. }\end{array}$ \\
\hline Ung/ 2013/ Australia [81] & 1 & $2000-2012$ & retrospective & III & $125(125 / 211)$ & $\begin{array}{l}\text { MMC }\left(12.5 \mathrm{mg} / \mathrm{m}^{2}\right) \text { for } 90 \mathrm{~min} \text { at } 42{ }^{\circ} \mathrm{C} \text { using coliseum } \\
\text { technique. }\end{array}$ \\
\hline Vaira/ 2010/ Italy [82] & 1 & $2002-2008$ & retrospective & III & $40(40 / 72)$ & $\begin{array}{l}\text { MMC }\left(16 \mathrm{mg} / \mathrm{m}^{2}\right)+\text { DDP }\left(100 \mathrm{mg} / \mathrm{m}^{2}\right) \text { for } 60 \mathrm{~min} \text { at } 41.5 \\
{ }^{\circ} \mathrm{C} \text { using semi-closed abdomen technique. } \\
\text { Before HIPEC, LV }\left(20 \mathrm{mg} / \mathrm{m}^{2}\right) \text { and } 5-\mathrm{FU}\left(400 \mathrm{mg} / \mathrm{m}^{2}\right) \\
\text { were delivered by systemic i.v. L-OHP }\left(460 \mathrm{mg} / \mathrm{m}^{2}\right) \text { for } \\
30 \mathrm{~min} \text { at } 42^{\circ} \mathrm{C} \text { using semi-closed abdomen technique. }\end{array}$ \\
\hline $\begin{array}{l}\text { van Leeuwen / 2008/ Sweden } \\
\text { [83] }\end{array}$ & 1 & 2003-2006 & retrospective & III & $38(38 / 103)$ & $\begin{array}{l}\text { Before HIPEC, LV }\left(30 \mathrm{mg} / \mathrm{m}^{2}\right) \text { and } 5-\mathrm{FU}\left(500 \mathrm{mg} / \mathrm{m}^{2}\right) \\
\text { were delivered by systemic i.v. } \\
\text { HIPEC: } \mathrm{L}-\mathrm{OHP}\left(460 \mathrm{mg} / \mathrm{m}^{2}\right) \text { for } 30 \mathrm{~min} \text { at } 42-44{ }^{\circ} \mathrm{C} \text { using } \\
\text { the coliseum technique. }\end{array}$ \\
\hline $\begin{array}{l}\text { van Oudheusden/ 2014/ } \\
\text { Netherlands [84] }\end{array}$ & 2 & 2005-2013 & retrospective & III & $113(113 / 149)$ & $\begin{array}{l}\text { MMC }\left(35 \mathrm{mg} / \mathrm{m}^{2}\right) \text { for } 90 \mathrm{~min} \text { at } 41-42^{\circ} \mathrm{C} \text { using coliseum } \\
\text { technique. }\end{array}$ \\
\hline $\begin{array}{l}\text { van Oudheusden / 2015/ } \\
\text { Netherlands [85] }\end{array}$ & 2 & 2005-2013 & retrospective & III & $252(252 / 351)$ & $\begin{array}{l}\text { MMC }\left(35 \mathrm{mg} / \mathrm{m}^{2}\right) \text { for } 90 \mathrm{~min} \text { at } 41.1^{\circ} \mathrm{C} \text { using open- } \\
\text { coliseum technique. }\end{array}$ \\
\hline Varban/ 2009/ America [86] & 1 & $1991-2007$ & retrospective & III & $128(128 / 142)$ & $\begin{array}{l}\text { MMC (total dose } 30 \mathrm{mg} \text { ) for } 60 \text { or } 90 \mathrm{~min} \text { at } 42.5^{\circ} \mathrm{C} \\
\text { using closed abdomen technique. } \\
\text { MMC (total dose } 40 \mathrm{mg} \text { ) for } 120 \mathrm{~min} \text { at } 42.5^{\circ} \mathrm{C} \text { using } \\
\text { closed abdomen technique. }\end{array}$ \\
\hline $\begin{array}{l}\text { Verwaal/ 2005/ Netherlands } \\
\text { 19] }\end{array}$ & 1 & $1995-2003$ & retrospective & III & $117(117 / 117)$ & $\begin{array}{l}\text { MMC }\left(35 \mathrm{mg} / \mathrm{m}^{2}\right) \text { for } 90 \mathrm{~min} \text { at } 40-41^{\circ} \mathrm{C} \text { using coliseum } \\
\text { technique. }\end{array}$ \\
\hline $\begin{array}{l}\text { Votanopoulos/2013/ America } \\
{[87]}\end{array}$ & 1 & 1993-2011 & retrospective & III & $217(217 / 217)$ & $\begin{array}{l}\text { MMC for } 90-120 \text { min at } 40.5-43{ }^{\circ} \mathrm{C} \text { using closed } \\
\text { abdomen technique. }\end{array}$ \\
\hline Winer/ 2014/ America [88] & 1 & 2001-2011 & retrospective & III & $30(30 / 67)$ & $\begin{array}{l}\text { MMC (total dose } 40 \mathrm{mg} \text { ) for } 100 \mathrm{~min} \text { at } 42^{\circ} \mathrm{C} \text { using } \\
\text { closed abdomen technique. }\end{array}$ \\
\hline $\begin{array}{l}\text { Witkamp/ 2001/ Netherlands } \\
\text { [89] }\end{array}$ & 1 & 1995-1997 & prospective & IIb & $29(29 / 29)$ & $\begin{array}{l}\text { MMC }\left(15-40 \mathrm{mg} / \mathrm{m}^{2} \text { initially; } 35 \mathrm{mg} / \mathrm{m}^{2} \text { majority) for } 90\right. \\
\text { min at } 40-41^{\circ} \mathrm{C} \text { using closed abdomen technique. }\end{array}$ \\
\hline Yan/ 2006/ Australia [90] & 1 & $1997-2006$ & prospective & IIb & $30(30 / 30)$ & $\begin{array}{l}\text { MMC }\left(10-12.5 \mathrm{mg} / \mathrm{m}^{2}\right) \text { for } 90 \mathrm{~min} \text { at } 42{ }^{\circ} \mathrm{C} \text { using } \\
\text { coliseum technique. }\end{array}$ \\
\hline Yan/ 2008/ Australia [91] & 1 & $1997-2007$ & retrospective & III & $50(50 / 50)$ & $\begin{array}{l}\text { MMC }\left(10-12.5 \mathrm{mg} / \mathrm{m}^{2}\right) \text { for } 90 \mathrm{~min} \text { at } 42{ }^{\circ} \mathrm{C} \text { using } \\
\text { coliseum technique. }\end{array}$ \\
\hline Zanon/ 2006/ Italy [92] & 1 & $1998-2004$ & prospective & III & $25(25 / 25)$ & $\begin{array}{l}\text { MMC }\left(15 \mathrm{mg} / \mathrm{m}^{2}\right) \text { for } 60 \mathrm{~min} \text { at } 42{ }^{\circ} \mathrm{C} \text { using closed } \\
\text { abdomen technique. }\end{array}$ \\
\hline
\end{tabular}

Note: MMC: mitomycin C; DDP: cisplatin; FU: fluorouracil; LV: leucovorin; L-OHP: oxaliplatin; CPT-11: irinotecan; NR: not reported

intraperitoneal therapy regimens, even targeted therapy, had interference on the survival outcomes in metaanalysis. The doubts were removed by the report of Kerscher et al [7]. In 2,406 CRC patients of no-PC and $\mathrm{PC}$, the survival outcomes for contemporary chemotherapy regimens (oxaliplatin or irinotecan) were compared with 5-FU regimens. For the CRC patients (without PC), survival outcomes for contemporary regimens were increased over 5-FU regimens (5-year survival rate 71.6\% vs. $63.3 \%, P=0.001)$. On the contrary, for patients with PC from CRC, the survival of L-OHP or irinotecan agent was similar to 5-FU regimens $(P>0.05)$, regardless of synchronous PC (2-year survival rate $31.1 \%$ vs. 19.1\%, $P=0.092$, and 5-year survival rate $20.8 \%$ vs. $5.8 \%, P=$ 0.081 ) or metachronous PC (2-year survival rate $71.5 \%$ vs. $58.5 \%, P=0.329$, and 5-year survival rate $28.1 \%$ vs. $24.4 \%, P=0.411)$.

There were a few statistical flaws in this metaanalysis. For example, only one RCT [12] was included. It may be due to the difficulty of performing RCT.
Therefore, we had to select meticulously current studies of best evidence level besides the only RCT. However, this meta-analysis showed acceptable outcomes of low heterogeneity and sensitivity. Regrettably, a patient-level (based on single patient data) meta-analysis as the gold standard for meta-analysis was not performed because of the difficulty in obtaining vast data from each database or institution. In addition to meta-analysis, this report provided a summary of 76 clinical studies published until today about CRS and HIPEC, which can get a review of published studies. In order to get the best evidence level results, more RCTs and prospective, multicenter, largescale clinical trials need to be performed in future studies.

Observing available data from 6 controlled studies (a total of 470patients) [12, 35, 36, 38, 39, 99], mortality or morbidity were found similar in both groups of HIPEC and traditional surgery, which was $4.3 \%$ vs. $5.0 \%$ and $19.8 \%$ vs. $19.5 \%$, respectively. The summarized HIPEC-related mortality and morbidity in 48 articles (the total number of patients, $n=4,809)[12,16,20,35,36,38,39$, 46, 48- 
Table 11: Summary of HIEPC-related procedures in different PC institutions or countries (published researches).

\begin{tabular}{|c|c|c|c|c|c|c|c|c|c|}
\hline \begin{tabular}{|l|} 
Country \\
/No. Institutions \\
\end{tabular} & Major Institutions & \begin{tabular}{|l|}
$\begin{array}{l}\text { No. } \\
\text { patients }\end{array}$ \\
\end{tabular} & Mode & \begin{tabular}{|l} 
HIPEC- \\
MMC alone
\end{tabular} & \begin{tabular}{|l} 
HIPEC- \\
MMC+DDP
\end{tabular} & \begin{tabular}{|l|}
$\begin{array}{l}\text { HIPEC-L-OHP } \\
\text { alone }\end{array}$ \\
\end{tabular} & \begin{tabular}{|l|}
$\begin{array}{l}\text { HIPEC- } \\
\text { other }\end{array}$ \\
\end{tabular} & \begin{tabular}{|l|} 
Temperature \\
$\left({ }^{\circ} \mathbf{C}\right)$
\end{tabular} & \begin{tabular}{|l|}
$\begin{array}{l}\text { Duration } \\
\text { (min) }\end{array}$ \\
\end{tabular} \\
\hline \multirow{20}{*}{ USA, 17} & \multicolumn{9}{|c|}{ Wake Forest University of Baptist Medical Center $[13,20,68,76,86,87]$} \\
\hline & Subtotal/Median/Range & $>709$ & $\mathrm{C}$ & $30 \mathrm{mg}$ & & & & $40.75(38.5-43)$ & $90(60-90)$ \\
\hline & \multicolumn{9}{|c|}{ University of Pittsburgh Medical Center (University of Pittsburgh) $[37,52,56,88]$} \\
\hline & Subtotal/Median/Range & 190 & $\mathrm{C}$ & $40 \mathrm{mg}$ & & & & $42(40-42)$ & \begin{tabular}{|ll}
100 & $(90$ \\
$100)$ & \\
\end{tabular} \\
\hline & \multicolumn{9}{|c|}{ Washington Hospital Center $[13,43,55]$} \\
\hline & Subtotal/Median/Range & $>81$ & $\mathrm{C}$ & $\begin{array}{l}10 \text { or } 12.5 \mathrm{mg} / \\
\mathrm{m}^{2}\end{array}$ & & & & $42(40-43)$ & $90(30-90)$ \\
\hline & \multicolumn{9}{|c|}{ Cancer Treatment Centers of America $[29,72]$} \\
\hline & Subtotal/Median/Range & ? & $\mathrm{O} / \mathrm{C}$ & Y & & Y & & $40-43$ & $30-120$ \\
\hline & \multicolumn{9}{|c|}{ Loma Linda University Medical Center $[29,72]$} \\
\hline & Subtotal/Median/Range & ? & $\mathrm{O} / \mathrm{C}$ & $\mathrm{Y}$ & & $\mathrm{Y}$ & & $40-43$ & $30-120$ \\
\hline & \multicolumn{9}{|c|}{ Medical College of Wisconsin $[29,72]$} \\
\hline & Subtotal/Median/Range & $?$ & $\mathrm{O} / \mathrm{C}$ & Y & & Y & & $40-43$ & $30-120$ \\
\hline & \multicolumn{9}{|c|}{ Mercy Medical Center $[29,72]$} \\
\hline & Subtotal/Median/Range & $?$ & $\mathrm{O} / \mathrm{C}$ & Y & & Y & & $40-43$ & $30-120$ \\
\hline & \multicolumn{9}{|c|}{ Moores Cancer Center, University of California [29, 72] } \\
\hline & Subtotal/Median/Range & $?$ & $\mathrm{O} / \mathrm{C}$ & $\mathrm{Y}$ & & Y & & $40-43$ & $30-120$ \\
\hline & \multicolumn{9}{|c|}{ Rutgers University $[29,72]$} \\
\hline & Subtotal/Median/Range & $?$ & $\mathrm{O} / \mathrm{C}$ & Y & & Y & & $40-43$ & $30-120$ \\
\hline & \multicolumn{9}{|l|}{ St Agnes Hospital $[15,34]$} \\
\hline & Subtotal/Median/Range & $>30$ & $\mathrm{O} / \mathrm{C}$ & $10-20 \mathrm{mg} / \mathrm{m}^{2}$ & & & & 42 & 90 \\
\hline
\end{tabular}

Note: C: closed abdomen technique for HIPEC; O: open abdomen technique for HIPEC; Y: yes; MMC: mitomycin C; DDP: cisplatin; 5-FU: fluorouracil; L-OHP: oxaliplatin; CPT-11: irinotecan

$50,52-54,56,58-62,64-69,71,73,75,76,79,80,82$ $90,92,100-104]$ were $2.8 \%(\mathrm{SD}, \pm 2.9 \%$; range, $0-12 \%)$ and $33.0 \%$ (SD, $\pm 13.4 \%$; range, $4-60 \%$ ), respectively. Some large-sample retrospective studies and populationbased analysis found a series of approximate results that the range of mortality was $2 \%-5.6 \%$ and morbidity was $25 \%-34 \%$ [93-97]. Furthermore, a systematic review of morbidity and mortality for CRS + HIPEC by Chua et al. [98] showed that the mortality and morbidity range from $0.9 \%$ to $5.8 \%$ and $12 \%$ to $52 \%$, respectively. Though evidence proved that safety for CRS+HIPEC was acceptable, a meta-analysis on mortality and mortality for CRS+HIPEC may be able to provide more convincing results on the mortality and morbidity.

With the summary of 76 studies, it is found that although HIPEC is now widely accepted and performed in most institutions, details of performing HIPEC varies among different institutions. As we noted, there are several mainly different techniques concerning HIPEC including 1) "open" or "closed" technique, 2) using MMC and/ or L-OHP, 3) mono-chemotherapy or combination of chemotherapy regimens, 4) temperature and duration of HIPEC. These can be further studied in future studies.
In conclusion, this meta-analysis showed that CRS+HIPEC comprehensive therapeutic strategy was associated with improvement of OS in CRC PC patients, and the results of the meta-analysis were proved of good reliability by low heterogeneous and robust sensitivity. Meanwhile, CRS and HIPEC can be performed with acceptable safety according to summary results of all 76 studies.

\section{MATERIALS AND METHODS}

\section{Search strategy}

The following databases were systematically searched up to July 31, 2016 including PubMed, Science Citation Index, EMBASE, and MEDLINE. The Cochrane Central Register of Controlled Trials, the National Institutes of Health trial registry, and conference proceeding articles from major oncologic and gastrointestinal cancer meetings were also sought for published results. The key words included 
Table 12: Summary of HIEPC-related procedures in different PC institutions or countries (published researches).

\begin{tabular}{|c|c|c|c|c|c|c|c|c|c|}
\hline $\begin{array}{l}\text { Country } \\
\text { /No. Institutions } \\
\end{array}$ & Major Institutions & $\begin{array}{l}\text { No. } \\
\text { patients }\end{array}$ & Mode & \begin{tabular}{|l|}
$\begin{array}{l}\text { HIPEC-MMC } \\
\text { alone }\end{array}$ \\
\end{tabular} & $\begin{array}{l}\text { HIPEC- } \\
\text { MMC+DDP }\end{array}$ & \begin{tabular}{|l|} 
HIPEC-L- \\
OHP alone \\
\end{tabular} & HIPEC-other & $\begin{array}{l}\text { Temperature } \\
\left({ }^{\circ} \mathrm{C}\right)\end{array}$ & $\begin{array}{l}\begin{array}{l}\text { Duration } \\
\text { (min) }\end{array} \\
\end{array}$ \\
\hline \multirow{11}{*}{ USA, 17} & \multicolumn{9}{|l|}{ St. John Hospital [29, 72] } \\
\hline & Subtotal/Median/Range & $?$ & $\mathrm{O} / \mathrm{C}$ & $\mathrm{Y}$ & & Y & & $40-43$ & $30-120$ \\
\hline & \multicolumn{9}{|l|}{ Tufts Medical Center $[29,72]$} \\
\hline & Subtotal/Median/Range & $?$ & $\mathrm{O} / \mathrm{C}$ & $\mathrm{Y}$ & & Y & & $40-43$ & $30-120$ \\
\hline & \multicolumn{9}{|l|}{ University of Illinois $[29,72]$} \\
\hline & Subtotal/Median/Range & $?$ & $\mathrm{O} / \mathrm{C}$ & Y & & Y & & $40-43$ & $30-120$ \\
\hline & \multicolumn{9}{|l|}{ University of Miami $[29,72]$} \\
\hline & Subtotal/Median/Range & $?$ & $\mathrm{O} / \mathrm{C}$ & $\mathrm{Y}$ & & Y & & $40-43$ & $30-120$ \\
\hline & $\begin{array}{lr}\text { American } & \text { Society of } \\
\text { Peritoneal } & \text { Surface } \\
\text { Malignancies } & \text { (ASPSM) } \\
{[29]} & \\
\end{array}$ & $?$ & NR & $\mathrm{Y}$ & & Y & others & NR & NR \\
\hline & $\begin{array}{l}\text { Mount Sinai Medical Center } \\
{[78]}\end{array}$ & 51 & $\mathrm{C}$ & $40 \mathrm{mg}$ & & & & $41-43$ & 90 \\
\hline & Sharp Health Care [13] & $?$ & $\mathrm{O} / \mathrm{C}$ & $\mathrm{Y}$ & $\mathrm{Y}$ & $\mathrm{Y}$ & $\begin{array}{l}\text { MMC+CPT-11, } \\
\text { 5-FU }\end{array}$ & $40-43$ & $30-90$ \\
\hline Subtotal & & $>1061$ & C & \begin{tabular}{|l|}
$30 / 40 \mathrm{mg}$ \\
$10-20 \mathrm{mg} / \mathrm{m} 2$ \\
10 or $12.5 \mathrm{mg} /$ \\
$\mathrm{m} 2$
\end{tabular} & & $\mathrm{Y}, 200 \mathrm{mg} / \mathrm{m} 2$ & $\begin{array}{l}\mathrm{MMC}+\mathrm{CPT}-11, \\
5-\mathrm{FU}\end{array}$ & $42(40-43)$ & $\begin{array}{l}90(60-90) \\
/ 30\end{array}$ \\
\hline \multirow[b]{2}{*}{ France, 14} & \multicolumn{9}{|c|}{ Centre Hospitalo-Universitaire Lyon Sud $[14,16,21,29,13,40,42,53,72,101]$} \\
\hline & Subtotal/Median/Range & $>500$ & $\mathrm{C}$ & $\begin{array}{l}10 \mathrm{mg} / \mathrm{L} \\
0.7 \mathrm{mg} / \mathrm{kg} \\
40-60 \mathrm{mg} \\
30-50 \mathrm{mg} / \mathrm{m}^{2}\end{array}$ & $\begin{array}{l}30-50 \mathrm{mg} / \mathrm{m}^{2}+ \\
50-100 \mathrm{mg} / \mathrm{m}^{2}\end{array}$ & $\begin{array}{l}360 \mathrm{mg} / \mathrm{m}^{2} \\
360-460 \quad \mathrm{mg} / \\
\mathrm{m}^{2}\end{array}$ & \begin{tabular}{|l|} 
MMC+CPT-11, \\
$5-F U$ \\
MMC $(0.7 \mathrm{mg} /$ \\
$\mathrm{kg})+\mathrm{CPT}-11$ \\
$\left(100 \mathrm{mg} / \mathrm{m}^{2}\right)$ \\
$\mathrm{L}-\mathrm{OHP}(360-$ \\
$\left.460 \mathrm{mg} / \mathrm{m}^{2}\right)+$ \\
$\mathrm{CPT}-11 \quad(100-$ \\
$\left.200 \mathrm{mg} / \mathrm{m}^{2}\right)$
\end{tabular} & $44(46-48) / 43$ & $\begin{array}{l}90(60-90) \\
130\end{array}$ \\
\hline
\end{tabular}

Note: C: closed abdomen technique for HIPEC; O: open abdomen technique for HIPEC; Y: yes; MMC: mitomycin C; DDP: cisplatin; 5-FU: fluorouracil; L-OHP: oxaliplatin; CPT-11: irinotecan

Table 13: Summary of HIEPC-related procedures in different $P C$ institutions or countries (published researches).

\begin{tabular}{|c|c|c|c|c|c|c|c|c|c|}
\hline $\begin{array}{l}\text { Country } \\
\text { /No. } \\
\text { Institutions }\end{array}$ & Major Institutions & $\begin{array}{l}\text { No. } \\
\text { patients }\end{array}$ & Mode & $\begin{array}{l}\text { HIPEC-MMC } \\
\text { alone }\end{array}$ & $\begin{array}{l}\text { HIPEC- } \\
\text { MMC+DDP }\end{array}$ & $\begin{array}{l}\text { HIPEC-L- } \\
\text { OHP alone }\end{array}$ & HIPEC-other & $\begin{array}{l}\text { Temperature } \\
\left({ }^{\circ} \mathrm{C}\right)\end{array}$ & $\begin{array}{l}\text { Duration } \\
\text { (min) }\end{array}$ \\
\hline \multirow{10}{*}{ France, 14} & \multicolumn{9}{|c|}{ Gustave Roussy Institute[13, 14, 28, 35, 36, 49, 50, 54, 73, 99-101] } \\
\hline & Subtotal/Median/Range & $>700$ & $\mathrm{O}$ & $\mid \begin{array}{l}5, \quad 8, \quad \text { or } \quad 10 \\
\mathrm{mg} / \mathrm{L} \\
20 \mathrm{mg} / \mathrm{m}^{2} \\
12.9+/-3.8 \mathrm{mg} / \\
\mathrm{m}^{2} \\
30-50 \mathrm{mg} / \mathrm{m}^{2}\end{array}$ & $\begin{array}{l}20 \mathrm{mg} / \mathrm{m}^{2}+200 \\
\mathrm{mg} / \mathrm{m}^{2} \\
30-50 \mathrm{mg} / \mathrm{m}^{2}+ \\
50-100 \mathrm{mg} / \mathrm{m}^{2}\end{array}$ & $\begin{array}{l}460 \mathrm{mg} / \mathrm{m}^{2} \\
360-460 \mathrm{mg} / \mathrm{m}^{2}\end{array}$ & $\begin{array}{l}\text { MMC+CPT-11, 5-FU } \\
\text { L-OHP }(360-460 \mathrm{mg} / \\
\left.\mathrm{m}^{2}\right)+ \text { CPT-11 }(100- \\
\left.200 \mathrm{mg} / \mathrm{m}^{2}\right) \\
\text { L-OHP }\left(300 \mathrm{mg} / \mathrm{m}^{2}\right) \\
+ \text { CPT-11 }(200 \mathrm{mg} / \\
\left.\mathrm{m}^{2}\right)\end{array}$ & $43(41-44) / 43$ & $\begin{array}{l}60(60-90) \\
/ 30\end{array}$ \\
\hline & \multicolumn{9}{|c|}{ Val d'Aurelle Center $[13,14,54,73]$} \\
\hline & Subtotal/Median/Range & $>66$ & $\mathrm{O}$ & $30-50 \mathrm{mg} / \mathrm{m}^{2}$ & $\begin{array}{l}30-50 \mathrm{mg} / \mathrm{m}^{2}+ \\
50-100 \mathrm{mg} / \mathrm{m}^{2}\end{array}$ & $\begin{array}{l}460 \mathrm{mg} / \mathrm{m}^{2} \\
360-460 \mathrm{mg} / \mathrm{m}^{2}\end{array}$ & $\begin{array}{l}\text { L-OHP }(360-460 \mathrm{mg} / \\
\left.\mathrm{m}^{2}\right)+ \text { CPT-11 }(100- \\
\left.200 \mathrm{mg} / \mathrm{m}^{2}\right) \\
\text { L-OHP }\left(300 \mathrm{mg} / \mathrm{m}^{2}\right) \\
+ \text { CPT-11 }(200 \mathrm{mg} / \\
\left.\mathrm{m}^{2}\right) \\
\end{array}$ & $43.5(40-43) / 43$ & $\begin{array}{l}60(60-90) \\
/ 30\end{array}$ \\
\hline & \multicolumn{9}{|c|}{ Centre Hospitalo-Universitaire l'Archet $[13,14,54]$} \\
\hline & Subtotal/Median/Range & $>25$ & $\mathrm{O} / \mathrm{C}$ & $30-50 \mathrm{mg} / \mathrm{m}^{2}$ & $\begin{array}{l}30-50 \mathrm{mg} / \mathrm{m}^{2}+ \\
50-100 \mathrm{mg} / \mathrm{m}^{2}\end{array}$ & $360-460 \mathrm{mg} / \mathrm{m}^{2}$ & $\begin{array}{l}\text { L-OHP }(360-460 \mathrm{mg} / \\
\left.\mathrm{m}^{2}\right)+\mathrm{CPT}-11(100- \\
\left.200 \mathrm{mg} / \mathrm{m}^{2}\right)\end{array}$ & $41.5(41-43) / 43$ & \begin{tabular}{|l}
$60(60-90)$ \\
130 or 60
\end{tabular} \\
\hline & \multicolumn{9}{|c|}{ Paul Papin Institute $[13,14,54]$} \\
\hline & Subtotal/Median/Range & $>25$ & $\mathrm{O} / \mathrm{C}$ & $30-50 \mathrm{mg} / \mathrm{m}^{2}$ & $\begin{array}{l}30-50 \mathrm{mg} / \mathrm{m}^{2}+ \\
50-100 \mathrm{mg} / \mathrm{m}^{2}\end{array}$ & $360-460 \mathrm{mg} / \mathrm{m}^{2}$ & $\begin{array}{l}\text { L-OHP }(360-460 \mathrm{mg} / \\
\left.\mathrm{m}^{2}\right)+\mathrm{CPT}-11(100- \\
\left.200 \mathrm{mg} / \mathrm{m}^{2}\right) \\
\end{array}$ & $41.5(41-43) / 43$ & $\begin{array}{l}60(60-90) \\
/ 30 \text { or } 60\end{array}$ \\
\hline & \multicolumn{9}{|c|}{ French Association of Surgery $[14,54]$} \\
\hline & Subtotal/Median/Range & ? & $\mathrm{O} / \mathrm{C}$ & $30-50 \mathrm{mg} / \mathrm{m}^{2}$ & $\begin{array}{l}30-50 \mathrm{mg} / \mathrm{m}^{2}+ \\
50-100 \mathrm{mg} / \mathrm{m}^{2}\end{array}$ & $360-460 \mathrm{mg} / \mathrm{m}^{2}$ & $\begin{array}{l}\mathrm{L}-\mathrm{OHP}(360-460 \mathrm{mg} / \\
\left.\mathrm{m}^{2}\right)+\mathrm{CPT}-11(100- \\
\left.200 \mathrm{mg} / \mathrm{m}^{2}\right)\end{array}$ & $41(41-43) / 43$ & $\begin{array}{lr}90 & (60- \\
120) & / 30 \\
\text { or } 60 & \end{array}$ \\
\hline
\end{tabular}

Note: C: closed abdomen technique for HIPEC; O: open abdomen technique for HIPEC; Y: yes; MMC: mitomycin C; DDP: cisplatin; 5-FU: fluorouracil; L-OHP: oxaliplatin; CPT-11: irinotecan 
Table 14: Summary of HIEPC-related procedures in different PC institutions or countries (published researches).

\begin{tabular}{|c|c|c|c|c|c|c|c|c|c|}
\hline \begin{tabular}{|l|} 
Country \\
/No. \\
Institutions \\
\end{tabular} & Major Institutions & No. patients & Mode & \begin{tabular}{|l|}
$\begin{array}{l}\text { HIPEC-MMC } \\
\text { alone }\end{array}$ \\
\end{tabular} & HIPEC-MMC+DDP & \begin{tabular}{|l}
$\begin{array}{l}\text { HIPEC-L-OHP } \\
\text { alone }\end{array}$ \\
\end{tabular} & HIPEC-other & \begin{tabular}{|l} 
Temperature \\
$\left({ }^{\circ} \mathrm{C}\right)$
\end{tabular} & $\begin{array}{l}\text { Duration } \\
(\min )\end{array}$ \\
\hline \multirow{10}{*}{ France, 14} & \multicolumn{9}{|l|}{ Hospital Lariboisiere $[29,72]$} \\
\hline & Subtotal/Median/Range & ? & $\mathrm{O} / \mathrm{C}$ & $\mathrm{Y}$ & & $\mathrm{Y}$ & & $40-43$ & $30-120$ \\
\hline & \multicolumn{9}{|c|}{ Louis-Mourier University Hospital $[62,62,101]$} \\
\hline & Subtotal/Median/Range & $>250$ & $\mathrm{O} / \mathrm{C}$ & $30-50 \mathrm{mg} / \mathrm{m}^{2}$ & $\begin{array}{l}30-50 \mathrm{mg} / \mathrm{m}^{2}+50-100 \\
\mathrm{mg} / \mathrm{m}^{2} \\
201 \mathrm{mg}+200 \mathrm{mg} / \mathrm{m}^{2}\end{array}$ & $360-460 \mathrm{mg} / \mathrm{m}^{2}$ & $\begin{array}{l}\text { L-OHP }(360-460 \mathrm{mg} / \\
\left.\mathrm{m}^{2}\right)+ \text { CPT- } 11(100- \\
\left.200 \mathrm{mg} / \mathrm{m}^{2}\right)\end{array}$ & \begin{tabular}{|l|}
42 \\
$148.5(47-50)$ \\
$/ 30-43$
\end{tabular} & $90(90-120) / 60$ \\
\hline & \begin{tabular}{|ll} 
Centre Hospitalier & de \\
Bellevue [13] & \\
\end{tabular} & 25 & $\mathrm{O} / \mathrm{C}$ & $\mathrm{Y}$ & $\mathrm{Y}$ & Y & MMC+CPT-11, 5-FU & $40-43$ & $30-90$ \\
\hline & \begin{tabular}{|lr} 
Centre & Hospitalo- \\
Universitaire Dijon [13]
\end{tabular} & 25 & $\mathrm{O} / \mathrm{C}$ & $\mathrm{Y}$ & $\mathrm{Y}$ & Y & MMC+CPT-11, 5-FU & $40-43$ & $30-90$ \\
\hline & Centre Jean Perrin [13] & 25 & $\mathrm{O} / \mathrm{C}$ & $\mathrm{Y}$ & $\mathrm{Y}$ & $\mathrm{Y}$ & MMC+CPT-11, 5-FU & $40-43$ & $30-90$ \\
\hline & CHU of Nice [48] & 74 & $\mathrm{O} / \mathrm{C}$ & 10 or $12.5 \mathrm{mg} / \mathrm{m}^{2}$ & & & & 43 & 90 \\
\hline & $\begin{array}{l}\text { Lyon Civil Hospices, South } \\
\text { Lyon University Hospital } \\
\text { Center [54] }\end{array}$ & ? & $\mathrm{O} / \mathrm{C}$ & $30-50 \mathrm{mg} / \mathrm{m}^{2}$ & $\begin{array}{l}30-50 \mathrm{mg} / \mathrm{m}^{2}+50-100 \\
\mathrm{mg} / \mathrm{m}^{2}\end{array}$ & $360-460 \mathrm{mg} / \mathrm{m}^{2}$ & \begin{tabular}{|l|} 
L-OHP $(360-460 \mathrm{mg} /$ \\
$\left.\mathrm{m}^{2}\right)+$ CPT- $11(100-$ \\
$\left.200 \mathrm{mg} / \mathrm{m}^{2}\right)$
\end{tabular} & $41-42.5,30-43$ & $90 / 60$ \\
\hline & $\begin{array}{l}\text { Université Claude Bernard } \\
\text { Lyon [13] }\end{array}$ & 25 & $\mathrm{O} / \mathrm{C}$ & $\mathrm{Y}$ & $\mathrm{Y}$ & $\mathrm{Y}$ & MMC+CPT-11, 5-FU & $40-43$ & $30-90$ \\
\hline Subtotal & & $>1038$ & $\mathrm{O}$ & $30-50 \mathrm{mg} / \mathrm{m} 2$ & $\begin{array}{l}30-50 \mathrm{mg} / \mathrm{m} 2+50- \\
100 \mathrm{mg} / \mathrm{m} 2\end{array}$ & $360-460 \mathrm{mg} / \mathrm{m} 2$ & \begin{tabular}{|l|} 
MMC+CPT-11, 5-FU \\
L-OHP + CPT-11 \\
MMC + CPT- 11 \\
\end{tabular} & $41.5(40-43) / 43$ & $\begin{array}{l}60(60-90) / 30 \\
\text { or } 60\end{array}$ \\
\hline \multirow{4}{*}{ Italy, 8} & \multicolumn{9}{|c|}{ National Cancer Institute of Milan $[29,46,72]$} \\
\hline & Subtotal/Median/Range & $?$ & $\mathrm{O} / \mathrm{C}$ & $\mathrm{Y}$ & \begin{tabular}{|l|}
$3.3 \mathrm{mg} / \mathrm{m}^{2} / \mathrm{L}+25 \mathrm{mg} /$ \\
$\mathrm{m}^{2} / \mathrm{L}$
\end{tabular} & $460 \mathrm{mg} / \mathrm{m}^{2}$ & & $42(41.5-43) / 43$ & $60(60-90) / 30$ \\
\hline & \multicolumn{9}{|c|}{ San Giuseppe Hospital $[13,46,82]$} \\
\hline & Subtotal/Median/Range & $>65$ & $\mathrm{O} / \mathrm{C}$ & $\mathrm{Y}$ & $\begin{array}{l}3.3 \mathrm{mg} / \mathrm{m}^{2} / \mathrm{L}+25 \mathrm{mg} / \\
\mathrm{m}^{2} / \mathrm{L} \\
16 \mathrm{mg} / \mathrm{m}^{2}+100 \mathrm{mg} / \mathrm{m}^{2}\end{array}$ & $460 \mathrm{mg} / \mathrm{m}^{2}$ & $\mathrm{MMC}+\mathrm{CPT}-11,5-\mathrm{FU}$ & $42(41.5-43) / 43$ & $60(60-90) / 30$ \\
\hline
\end{tabular}

Note: C: closed abdomen technique for HIPEC; O: open abdomen technique for HIPEC; Y: yes; MMC: mitomycin C; DDP: cisplatin; 5-FU: fluorouracil; L-OHP: oxaliplatin; CPT-11: irinotecan

Table 15: Summary of HIEPC-related procedures in different PC institutions or countries (published researches).

\begin{tabular}{|c|c|c|c|c|c|c|c|c|c|}
\hline $\begin{array}{l}\text { Country } \\
\text { /No. } \\
\text { Institutions }\end{array}$ & Major Institutions & $\begin{array}{l}\text { No. } \\
\text { patients }\end{array}$ & Mode & $\begin{array}{l}\text { HIPEC- } \\
\text { MMC } \\
\text { alone }\end{array}$ & $\begin{array}{l}\text { HIPEC- } \\
\text { MMC+DDP }\end{array}$ & $\begin{array}{l}\text { HIPEC-L- } \\
\text { OHP alone }\end{array}$ & HIPEC-other & $\begin{array}{l}\text { Temperature } \\
\left({ }^{\circ} \mathrm{C}\right)\end{array}$ & $\begin{array}{l}\text { Duration } \\
(\mathrm{min})\end{array}$ \\
\hline \multirow{8}{*}{ Italy, 8} & \multicolumn{9}{|c|}{ Regina Elena National Cancer Institute $[13,46]$} \\
\hline & Subtotal/Median/Range & $>25$ & $\mathrm{O} / \mathrm{C}$ & Y & $\begin{array}{l}3.3 \mathrm{mg} / \mathrm{m}^{2} / \mathrm{L}+25 \\
\mathrm{mg} / \mathrm{m}^{2} / \mathrm{L}\end{array}$ & $460 \mathrm{mg} / \mathrm{m}^{2}$ & $\begin{array}{l}\text { MMC+CPT-11, } \\
\text { 5-FU }\end{array}$ & \begin{tabular}{|ll}
42 & $(41.5-43)$ \\
$/ 43$ & \\
\end{tabular} & $\begin{array}{l}60(60-90) \\
/ 30\end{array}$ \\
\hline & \multicolumn{9}{|l|}{ University of Padua $[46,71]$} \\
\hline & Subtotal/Median/Range & $>46$ & $\mathrm{O} / \mathrm{C}$ & $3.3 \mathrm{mg} / \mathrm{m}^{2} / \mathrm{L}$ & $\begin{array}{l}3.3 \mathrm{mg} / \mathrm{m}^{2} / \mathrm{L}+25 \\
\mathrm{mg} / \mathrm{m}^{2} / \mathrm{L}\end{array}$ & $460 \mathrm{mg} / \mathrm{m}^{2}$ & & $\begin{array}{|ll|}42 & (41.5-43) \\
/ 43 & \\
\end{array}$ & $\begin{array}{l}90(60-90) \\
/ 33\end{array}$ \\
\hline & Istituto Nazional Tumori [13 & 25 & $\mathrm{O} / \mathrm{C}$ & Y & $\mathrm{Y}$ & Y & $\begin{array}{l}\text { MMC+CPT-11, } \\
\text { 5-FU }\end{array}$ & $40-43$ & $30-90$ \\
\hline & Ospedale di Bentivoglio [46] & $?$ & $\mathrm{O} / \mathrm{C}$ & & $\begin{array}{l}3.3 \mathrm{mg} / \mathrm{m}^{2} / \mathrm{L}+25 \\
\mathrm{mg} / \mathrm{m}^{2} / \mathrm{L}\end{array}$ & $460 \mathrm{mg} / \mathrm{m}^{2}$ & & $41.5-43 / 43$ & $60-90 / 30$ \\
\hline & $\begin{array}{l}\text { San Camillo-Forlanini Hospital } \\
{[46]}\end{array}$ & ? & $\mathrm{O} / \mathrm{C}$ & & $\begin{array}{l}3.3 \mathrm{mg} / \mathrm{m}^{2} / \mathrm{L}+25 \\
\mathrm{mg} / \mathrm{m}^{2} / \mathrm{L}\end{array}$ & $460 \mathrm{mg} / \mathrm{m}^{2}$ & & $41.5-43 / 43$ & $60-90 / 30$ \\
\hline & $\begin{array}{l}\text { San Giovanni Battista Antica } \\
\text { Sede Hospital [92] }\end{array}$ & 25 & $\mathrm{C}$ & $15 \mathrm{mg} / \mathrm{m}^{2}$ & & & & 42 & 60 \\
\hline Subtotal & & $>186$ & $\mathrm{C}$ & Y & $\begin{array}{l}3.3 \mathrm{mg} / \mathrm{m} 2 / \mathrm{L}+25 \\
\mathrm{mg} / \mathrm{m} 2 / \mathrm{L}\end{array}$ & $460 \mathrm{mg} / \mathrm{m} 2$ & $\begin{array}{l}\text { MMC+CPT-11, } \\
\text { 5-FU }\end{array}$ & \begin{tabular}{|ll}
42 & $(41.5-43)$ \\
$/ 43$ & \\
\end{tabular} & $\begin{array}{l}60(60-90) \\
/ 30\end{array}$ \\
\hline \multirow{6}{*}{ Belgium, 6} & \multicolumn{9}{|l|}{ Jolimont Hospital $[14,54,58]$} \\
\hline & Subtotal/Median/Range & $?$ & $\mathrm{O}$ & $\begin{array}{l}30-50 \mathrm{mg} / \\
\mathrm{m}^{2}\end{array}$ & $\begin{array}{l}30-50 \mathrm{mg} / \mathrm{m}^{2}+50- \\
100 \mathrm{mg} / \mathrm{m}^{2}\end{array}$ & $\begin{array}{l}360-460 \mathrm{mg} / \\
\mathrm{m}^{2} \\
460 \mathrm{mg} / \mathrm{m}^{2}\end{array}$ & $\begin{array}{l}\mathrm{L}-\mathrm{OHP}(360-460 \\
\left.\mathrm{mg} / \mathrm{m}^{2}\right)+\mathrm{CPT}- \\
11(100-200 \mathrm{mg} / \\
\left.\mathrm{m}^{2}\right)\end{array}$ & $\begin{array}{l}41.5(41-42.5) \\
/ 43\end{array}$ & $\begin{array}{lr}90 & (60- \\
120) & / 30 \\
\text { or } 60 & \end{array}$ \\
\hline & \multicolumn{9}{|l|}{ Ghent University Hospital $[47,58]$} \\
\hline & Subtotal/Median/Range & $>152$ & $\mathrm{O}$ & $35 \mathrm{mg} / \mathrm{m}^{2}$ & & $460 \mathrm{mg} / \mathrm{m}^{2}$ & & $41(41-42)$ & $\begin{array}{l}60(60-90) \\
/ 30\end{array}$ \\
\hline & \multicolumn{9}{|c|}{ University Hospitals Gasthuisberg $[58,59]$} \\
\hline & Subtotal/Median/Range & $>39$ & $\mathrm{O}$ & & & $460 \mathrm{mg} / \mathrm{m}^{2}$ & & $41-42$ & 30 \\
\hline
\end{tabular}

Note: C: closed abdomen technique for HIPEC; O: open abdomen technique for HIPEC; Y: yes; MMC: mitomycin C; DDP: cisplatin; 5-FU: fluorouracil; L-OHP: oxaliplatin; CPT-11: irinotecan 
Table 16: Summary of HIEPC-related procedures in different PC institutions or countries (published researches).

\begin{tabular}{|c|c|c|c|c|c|c|c|c|c|}
\hline $\begin{array}{l}\text { Country } \\
\text { /No. Institutions }\end{array}$ & Major Institutions & \begin{tabular}{|l|}
$\begin{array}{l}\text { No. } \\
\text { patients }\end{array}$ \\
\end{tabular} & Mode & $\begin{array}{l}\text { HIPEC- } \\
\text { MMC alone }\end{array}$ & $\begin{array}{l}\text { HIPEC- } \\
\text { MMC+DDP }\end{array}$ & $\begin{array}{l}\text { HIPEC-L- } \\
\text { OHP alone }\end{array}$ & HIPEC-other & \begin{tabular}{|l|} 
Temperature \\
$\left({ }^{\circ} \mathbf{C}\right)$ \\
\end{tabular} & \begin{tabular}{|l|}
$\begin{array}{l}\text { Duration } \\
\text { (min) }\end{array}$ \\
\end{tabular} \\
\hline \multirow{3}{*}{ Belgium, 6} & $\begin{array}{|lr|}\text { I-Biostat, } & \text { Katholieke } \\
\text { Universiteit } \quad \text { Leuven and } \\
\text { Universiteit Hasselt [58] }\end{array}$ & $?$ & $\mathrm{O}$ & & & $460 \mathrm{mg} / \mathrm{m}^{2}$ & & $41-42$ & 30 \\
\hline & UCL Mont-Godinne [58] & ? & $\mathrm{O}$ & & & $460 \mathrm{mg} / \mathrm{m}^{2}$ & & $41-42$ & 30 \\
\hline & Ziekenhuis Oost-Limburg [58] & $?$ & $\mathrm{O}$ & & & $460 \mathrm{mg} / \mathrm{m}^{2}$ & & $41-42$ & 30 \\
\hline Subtotal & & $>191$ & $\mathrm{O}$ & $30-50 \mathrm{mg} / \mathrm{m} 2$ & $\begin{array}{l}30-50 \mathrm{mg} / \mathrm{m} 2 \\
+50-100 \mathrm{mg} / \\
\mathrm{m} 2\end{array}$ & $460 \mathrm{mg} / \mathrm{m} 2$ & $\begin{array}{l}\mathrm{L}-\mathrm{OHP}+\mathrm{CPT}- \\
11\end{array}$ & 41 /41-42 $(41-42)$ & $\begin{array}{l}90(60-90) \\
130 \text { or } 60\end{array}$ \\
\hline \multirow{9}{*}{ Netherlands, 6} & \multicolumn{9}{|c|}{ Netherlands Cancer Institute $[12,19,51,59,65,66,77,89]$} \\
\hline & Subtotal/Median/Range & 863 & $\mathrm{O}$ & $35 \mathrm{mg} / \mathrm{m}^{2}$ & & & & $41.5(41-42)$ & 90 \\
\hline & \multicolumn{9}{|c|}{ Catharina Hospital Eindhoven $[44,63-65,84,85,102]$} \\
\hline & Subtotal/Median/Range & $>300$ & $\mathrm{O}$ & $35 \mathrm{mg} / \mathrm{m}^{2}$ & & & & $41.5(41-42)$ & 90 \\
\hline & \multicolumn{9}{|c|}{ Sint Antonius Hospital Nieuwegein $[44,65,84,85]$} \\
\hline & Subtotal/Median/Range & $>121$ & $\mathrm{O}$ & $35 \mathrm{mg} / \mathrm{m}^{2}$ & & & & $41.5(41-42)$ & 90 \\
\hline & $\begin{array}{l}\text { Radboud University Nijmegen } \\
\text { Medical Center [64] }\end{array}$ & 12 & $\mathrm{O}$ & $35 \mathrm{mg} / \mathrm{m}^{2}$ & & & & $41-42$ & 90 \\
\hline & $\begin{array}{|ll|}\text { University Medical Center } \\
\text { Groningen [64] }\end{array}$ & 48 & $\mathrm{O}$ & $35 \mathrm{mg} / \mathrm{m}^{2}$ & & & & $41-42$ & 90 \\
\hline & \begin{tabular}{|l|} 
VU Medical Centre Amsterdam \\
{$[64]$}
\end{tabular} & 17 & 0 & $35 \mathrm{mg} / \mathrm{m}^{2}$ & & & & $41-42$ & 90 \\
\hline Subtotal & & $>1432$ & $\mathrm{O}$ & $35 \mathrm{mg} / \mathrm{m} 2$ & & & & $41.5(41-42)$ & 90 \\
\hline \multirow{4}{*}{$\begin{array}{l}\text { Spain } \\
6\end{array}$} & \multicolumn{9}{|l|}{ Hospital San Jaime $[29,71]$} \\
\hline & Subtotal/Median/Range & $?$ & $\mathrm{O} / \mathrm{C}$ & $\mathrm{Y}$ & & Y & & $40-43$ & $30-120$ \\
\hline & \multicolumn{9}{|l|}{ Hospital Torrecardenas $[29,71]$} \\
\hline & Subtotal/Median/Range & ? & $\mathrm{O} / \mathrm{C}$ & $\mathrm{Y}$ & & $\mathrm{Y}$ & & $40-43$ & $30-120$ \\
\hline
\end{tabular}

Note: C: closed abdomen technique for HIPEC; O: open abdomen technique for HIPEC; Y: yes; MMC: mitomycin C; DDP: cisplatin; 5-FU: fluorouracil; L-OHP: oxaliplatin; CPT-11: irinotecan

Table 17: Summary of HIEPC-related procedures in different PC institutions or countries (published researches).

\begin{tabular}{|c|c|c|c|c|c|c|c|c|c|}
\hline $\begin{array}{l}\text { Country } \\
\text { /No. } \\
\text { Institutions }\end{array}$ & Major Institutions & $\begin{array}{l}\text { No. } \\
\text { patients }\end{array}$ & Mode & $\begin{array}{l}\text { HIPEC- } \\
\text { MMC alone }\end{array}$ & $\begin{array}{l}\text { HIPEC- } \\
\text { MMC+DDP }\end{array}$ & $\begin{array}{l}\text { HIPEC-L- } \\
\text { OHP alone }\end{array}$ & HIPEC-other & $\begin{array}{l}\text { Temperature } \\
\left({ }^{\circ} \mathrm{C}\right)\end{array}$ & $\begin{array}{l}\text { Duration } \\
\text { (min) }\end{array}$ \\
\hline \multirow{6}{*}{ Spain, 6} & \multicolumn{9}{|l|}{ M. D. Anderson Cancer Center $[29,71]$} \\
\hline & Subtotal/Median/Range & $?$ & $\mathrm{O} / \mathrm{C}$ & $\mathrm{Y}$ & & $\mathrm{Y}$ & & $40-43$ & $30-120$ \\
\hline & \multicolumn{9}{|l|}{ San Jose Hospital $[29,71]$} \\
\hline & Subtotal/Median/Range & $?$ & $\mathrm{O} / \mathrm{C}$ & $\mathrm{Y}$ & & $\mathrm{Y}$ & & $40-43$ & $30-120$ \\
\hline & Hospital Infanta Cristina [71] & $?$ & $\mathrm{O} / \mathrm{C}$ & $\mathrm{Y}$ & & $\mathrm{Y}$ & others & $40-43$ & $30-120$ \\
\hline & Hospital Santiago Apostol [13] & 25 & $\mathrm{O} / \mathrm{C}$ & $\mathrm{Y}$ & $\mathrm{Y}$ & Y & $\begin{array}{l}\mathrm{MMC}+\mathrm{CPT}-11, \\
5-\mathrm{FU}\end{array}$ & $40-43$ & $30-90$ \\
\hline Subtotal & & $>25$ & $\mathrm{O} / \mathrm{C}$ & $\mathrm{Y}$ & & $\mathrm{Y}$ & $\begin{array}{l}\mathrm{MMC}+\mathrm{CPT}-11, \\
5-\mathrm{FU}\end{array}$ & $41.5(40-43)$ & $\begin{array}{ll}90 & (30- \\
120) / 30\end{array}$ \\
\hline \multirow{4}{*}{ Canada, 2} & \multicolumn{9}{|l|}{ University of Calgary $[56,66,68,73]$} \\
\hline & Subtotal/Median/Range & 375 & $\mathrm{O}$ & $12-15 \mathrm{mg}$ & & $400 \mathrm{mg} / \mathrm{m}^{2}$ & & $41.5(40-42)$ & 60 \\
\hline & \multicolumn{9}{|c|}{ Maisonneuve-Rosemont Hospital, University of Montreal [14, 38, 53] } \\
\hline & Subtotal/Median/Range & $>40$ & $\mathrm{O}$ & $30-50 \mathrm{mg} / \mathrm{m}^{2}$ & $\begin{array}{l}30-50 \mathrm{mg} / \mathrm{m}^{2}+ \\
50-100 \mathrm{mg} / \mathrm{m}^{2}\end{array}$ & $\begin{array}{l}360-460 \mathrm{mg} / \\
\mathrm{m}^{2}\end{array}$ & $\begin{array}{l}\mathrm{L}-\mathrm{OHP}(360- \\
\left.460 \mathrm{mg} / \mathrm{m}^{2}\right)+ \\
\mathrm{CPT}-11(100- \\
\left.200 \mathrm{mg} / \mathrm{m}^{2}\right) \\
\end{array}$ & $\begin{array}{l}41.5(41-42.5) \\
/ 43(42-43)\end{array}$ & $\begin{array}{l}90 \quad(60- \\
120) \\
\text { or } 60\end{array}$ \\
\hline Subtotal & & $>415$ & $\mathrm{O}$ & $\begin{array}{l}12-15 \quad \mathrm{mg} \\
/ 30-50 \mathrm{mg} / \mathrm{m}^{2}\end{array}$ & $\begin{array}{l}30-50 \mathrm{mg} / \mathrm{m}^{2}+ \\
50-100 \mathrm{mg} / \mathrm{m}^{2}\end{array}$ & $\begin{array}{l}360-460 \mathrm{mg} / \\
\mathrm{m}^{2}\end{array}$ & $\begin{array}{l}\text { L-OHP + CPT- } \\
11\end{array}$ & $\begin{array}{l}41.5(41-42.5) \\
/ 43(42-43)\end{array}$ & $\begin{array}{l}60 \text { or } 90 \\
(60-120) \\
/ 30 \text { or } 60\end{array}$ \\
\hline \multirow{3}{*}{ Greece, 2} & \multicolumn{9}{|l|}{ Metaxa Cancer Memorial Hospital $[29,71]$} \\
\hline & Subtotal/Median/Range & $?$ & $\mathrm{O} / \mathrm{C}$ & Y & & $\mathrm{Y}$ & & $40-43$ & $30-120$ \\
\hline & Didimotichon General Hospital [13] & 25 & $\mathrm{O} / \mathrm{C}$ & $\mathrm{Y}$ & $\mathrm{Y}$ & $\mathrm{Y}$ & $\begin{array}{l}\mathrm{MMC}+\mathrm{CPT}-11, \\
\text { 5-FU }\end{array}$ & $40-43$ & $30-90$ \\
\hline Subtotal & & $>25$ & $\mathrm{O} / \mathrm{C}$ & $\mathrm{Y}$ & $\mathrm{Y}$ & Y & $\begin{array}{l}\mathrm{MMC}+\mathrm{CPT}-11 \\
5-\mathrm{FU}\end{array}$ & $41.5(40-43)$ & $30-90$ \\
\hline Australia, 1 & \multicolumn{9}{|c|}{ St. George Hospital $[15,26,29,34,41,45,64,72,81,90,91]$} \\
\hline Subtotal & & $>618$ & $\mathrm{O}$ & $\begin{array}{l}10-12.5 \mathrm{mg} / \\
\mathrm{m} 2\end{array}$ & & $350 \mathrm{mg} / \mathrm{m}^{2}$ & & 42 & 90 or 30 \\
\hline
\end{tabular}

Note: C: closed abdomen technique for HIPEC; O: open abdomen technique for HIPEC; Y: yes; MMC: mitomycin C; DDP: cisplatin; 5-FU: fluorouracil; L-OHP: oxaliplatin; CPT-11: irinotecan 
Table 18: Summary of HIEPC-related procedures in different PC institutions or countries (published researches).

\begin{tabular}{|c|c|c|c|c|c|c|c|c|c|}
\hline $\begin{array}{l}\text { Country } \\
\text { /No. Institutions }\end{array}$ & Major Institutions & $\begin{array}{l}\text { No. } \\
\text { patients }\end{array}$ & Mode & \begin{tabular}{|l|} 
HIPEC- \\
MMC \\
alone \\
\end{tabular} & $\begin{array}{l}\text { HIPEC- } \\
\text { MMC+DDP }\end{array}$ & \begin{tabular}{|l|} 
HIPEC- \\
L-OHP \\
alone \\
\end{tabular} & HIPEC-other & $\begin{array}{l}\text { Temperature } \\
\left({ }^{\circ} \mathrm{C}\right)\end{array}$ & $\begin{array}{l}\text { Duration } \\
\text { (min) }\end{array}$ \\
\hline China, 1 & \multicolumn{9}{|c|}{ Zhongnan Hospital of Wuhan University [39] } \\
\hline Subtotal & & 62 & $\mathrm{O}$ & & $\begin{array}{l}\text { MMC (30 mg) } \\
+\quad \text { DDP (120 } \\
\text { mg) }\end{array}$ & & & $43.0 \pm 0.5$ & 90 \\
\hline Norway, 1 & \multicolumn{9}{|c|}{ Norwegian Radium Hospital [103] } \\
\hline Subtotal & & 109 & $\mathrm{O} / \mathrm{C}$ & $35 \mathrm{mg} / \mathrm{m} 2$ & & & & $41.4(39.5-42.1)$ & 90 \\
\hline Denmark, 1 & \multicolumn{9}{|c|}{ Aarhus University Hospital $[60,75]$} \\
\hline Subtotal & & 53 & $\mathrm{O}$ & $35 \mathrm{mg} / \mathrm{m} 2$ & & & & $41-42.5$ & 90 \\
\hline Germany, 1 & \multicolumn{9}{|c|}{ University of Wuerzburg Medical Centre $[15,29,72]$} \\
\hline Subtotal & & $>11$ & 0 & $\begin{array}{l}10-20 \mathrm{mg} / \\
\mathrm{m} 2\end{array}$ & & $\mathrm{Y}$ & & $42 / 40-43$ & $90 / 30$ \\
\hline Israel. 1 & \multicolumn{9}{|c|}{ Tel Aviv Medical Center [13] } \\
\hline Subtotal & & 25 & $\mathrm{O} / \mathrm{C}$ & $\mathrm{Y}$ & $\mathrm{Y}$ & Y & $\begin{array}{l}\mathrm{MMC}+\mathrm{CPT}-11, \\
\text { 5-FU }\end{array}$ & $40-43$ & $30-90$ \\
\hline Japan, 1 & \multicolumn{9}{|c|}{ Shizuoka Cancer Centre [13] } \\
\hline Subtotal & & 25 & $\mathrm{O} / \mathrm{C}$ & $\mathrm{Y}$ & Y & $\mathrm{Y}$ & $\begin{array}{l}\text { MMC+CPT-11, } \\
\text { 5-FU }\end{array}$ & $40-43$ & $30-90$ \\
\hline Mexico, 1 & \multicolumn{9}{|c|}{ Instituto Jalisciense de Cancerologia $[29,72]$} \\
\hline Subtotal & & $?$ & $\mathrm{O} / \mathrm{C}$ & Y & & $\mathrm{Y}$ & & $40-43$ & $30-120$ \\
\hline $\begin{array}{l}\text { Serbia and } \\
\text { Montenegro, } 1\end{array}$ & \multicolumn{9}{|c|}{ First Surgical University Hospital, Clinical Center of Serbia [61] } \\
\hline Subtotal & & 18 & C & $\begin{array}{l}10 \text { or } 12.5 \\
\mathrm{mg} / \mathrm{m} 2\end{array}$ & & & & 42 & 120 \\
\hline
\end{tabular}

Note: C: closed abdomen technique for HIPEC; O: open abdomen technique for HIPEC; Y: yes; MMC: mitomycin C; DDP: cisplatin; 5-FU: fluorouracil; L-OHP: oxaliplatin; CPT-11: irinotecan

Table 19: Summary of HIEPC-related procedures in different PC institutions or countries (published researches).

\begin{tabular}{|c|c|c|c|c|c|c|c|c|c|}
\hline $\begin{array}{l}\text { Country } \\
\text { /No. Institutions }\end{array}$ & $\begin{array}{l}\text { Major } \\
\text { Institutions }\end{array}$ & \begin{tabular}{|l|} 
No. \\
patients
\end{tabular} & Mode & $\begin{array}{l}\text { HIPEC- } \\
\text { MMC alone }\end{array}$ & $\begin{array}{l}\text { HIPEC- } \\
\text { MMC+DDP }\end{array}$ & \begin{tabular}{|l|} 
HIPEC-L- \\
OHP alone
\end{tabular} & HIPEC-other & \begin{tabular}{|l} 
Temperature \\
$\left({ }^{\circ} \mathbf{C}\right)$
\end{tabular} & \begin{tabular}{|l}
$\begin{array}{l}\text { Duration } \\
\text { (min) }\end{array}$ \\
\end{tabular} \\
\hline Serbia, 1 & \multicolumn{9}{|c|}{ Institute for Oncology and Radiology of Serbia [70] } \\
\hline Subtotal & & 61 & $\mathrm{C}$ & & & $410 \mathrm{mg} / \mathrm{m} 2$ & & 41 & $30-60$ \\
\hline Singapore, 1 & \multicolumn{9}{|c|}{ National Cancer Centre Singapore $[79,81]$} \\
\hline Subtotal & & 63 & $\mathrm{C}$ & $\mathrm{Y}$ & & & & 42 & 60 \\
\hline Sweden, 1 & \multicolumn{9}{|c|}{ Akademiska Sjukhuset, Uppsala University Hospital [83] } \\
\hline Subtotal & & 38 & $\mathrm{O}$ & & & $460 \mathrm{mg} / \mathrm{m} 2$ & & $42-44$ & 30 \\
\hline Total & 73 & $\approx 6,500$ & $\begin{array}{l}\mathrm{O} \\
(\mathrm{n}=63) \\
\mathrm{C} \\
(\mathrm{n}=51)\end{array}$ & $\begin{array}{l}\mathrm{n}=64 \\
30-50 \mathrm{mg} / \mathrm{m} 2 \\
10-12.5 \mathrm{mg} / \\
\mathrm{m}^{2} \\
35 \mathrm{mg} / \mathrm{m}^{2} \\
10-20 \mathrm{mg} / \mathrm{m}^{2}\end{array}$ & $\begin{array}{l}\mathrm{n}=24 \\
30-50 \mathrm{mg} / \mathrm{m}^{2} \\
+50-100 \mathrm{mg} / \\
\mathrm{m}^{2}\end{array}$ & $\begin{array}{l}\mathrm{n}=43 \\
460 \mathrm{mg} / \mathrm{m} 2 \\
360-460 \mathrm{mg} / \\
\mathrm{m}^{2}\end{array}$ & $\begin{array}{l}\text { MMC+CPT-11, } \\
5-\mathrm{FU} \\
\text { L-OHP + CPT- } \\
11 \\
\text { MMC + CPT-11 }\end{array}$ & $\begin{array}{l}41.5 \quad(40-43) \\
/ 43(40-43)\end{array}$ & $\begin{array}{l}90(60-90) \\
/ 60\end{array}$ \\
\hline
\end{tabular}

Note: C: closed abdomen technique for HIPEC; O: open abdomen technique for HIPEC; Y: yes; MMC: mitomycin C; DDP: cisplatin; 5-FU: fluorouracil; L-OHP: oxaliplatin; CPT-11: irinotecan

“colon", "rectum", “colorectal", “cancer", “peritoneal carcinomatosis", "hyperthermic intraperitoneal chemotherapy", and synonyms and related terms for these words. The MeSH terms included "colon cancer", "rectal cancer", "colorectal cancer", "peritoneal carcinomatosis", "hyperthermic chemotherapy", "hyperthermic intraperitoneal chemotherapy", "HIPEC", "intraperitoneal chemohyperthermia", and "IPCH". The combined application of "key words terms" and "MeSH terms" were conducted to improve the efficiency and accuracy of literature search.

\section{Selection criteria}

For inclusion in the meta-analysis and summarized HIPEC-related data analysis, a study had to fulfill the following criteria: (1) According to the North-England evidence-based guidelines [105, 106], excluded from IV levels evidence of literatures were included; (2) All patients were diagnosed CRC PC; (3) For assessing $\mathrm{CRS}+\mathrm{HIPEC} \pm \mathrm{SC} / \mathrm{EPIC}$, the intervening measure group was CRS+HIPEC \pm SC/EPIC, while the control group was traditional therapy of surgery and/or SC; For systematic review of CRS+HIPEC to treat CRC PC, HIPEC-related literatures involving clinical efficacy evaluation were 
Table 20: Survival of Patients with CRC PC Treated by CRS and HIPEC and/or EPIC and/or SC: Summary of 76 Researches.

\begin{tabular}{|c|c|c|c|c|c|c|c|c|c|c|c|c|}
\hline $\begin{array}{l}\text { Author/ Years/ } \\
\text { Country }\end{array}$ & $\begin{array}{ll}1-\mathrm{yr} \\
(\%)\end{array} \quad$ SR & 2-yr SR (\%) & 3-yr SR (\%) & 4-yr SR (\%) & $\begin{array}{ll}5-\mathrm{yr} & \text { SR } \\
(\%) & \end{array}$ & $\begin{array}{l}\text { Mortality } \\
\text { Rate (\%) }\end{array}$ & $\begin{array}{l}\text { Morbidity } \\
\text { Rate (\%) }\end{array}$ & $\begin{array}{l}\text { Median OS } \\
\text { (mo) }\end{array}$ & $\begin{array}{l}\text { OS 95\% CI } \\
\text { (mo) }\end{array}$ & $\begin{array}{l}\text { PFS }(95 \% \\
\text { CI) (mo) }\end{array}$ & 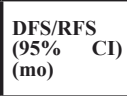 & \begin{tabular}{|l} 
Follow- \\
up times \\
(range) \\
(mo)
\end{tabular} \\
\hline \multicolumn{13}{|l|}{ Controlled Studies } \\
\hline $\begin{array}{lll}\text { Chua } \quad \mathrm{TC} / & 2009 / \\
\text { Australia [34] } & \end{array}$ & $\approx 84$ & $\approx 50$ & $\approx 26$ & NA & NA & NR & NR & 13 & NR & NR & NR & $18(9-59)$ \\
\hline $\begin{array}{ll}\text { Chua TC/ } & \text { 2011/ } \\
\text { Australia [15] } & \end{array}$ & 92 & NR & 55 & NR & 30 & NR & NR & 38 & $30.2-45.2$ & NR & $\begin{array}{l}17 \text { (1-216) } \\
\text { (two groups) }\end{array}$ & $\begin{array}{l}17 \\
126)\end{array}$ \\
\hline $\begin{array}{ll}\text { Chua TC/ } & \text { 2013/ } \\
\text { Australia }[26] & \end{array}$ & NR & NR & NR & NR & 41 & NR & NR & 38 & $21.1-54.9$ & NR & $\begin{array}{l}33 \\
43.8) \\
(\mathrm{RFS})\end{array}$ & $22(5-88)$ \\
\hline $\begin{array}{l}\text { Elias D/ 2001/ France } \\
\text { [35] }\end{array}$ & NR & $\approx 70$ & $\approx 53$ & $\approx 53$ & $\approx 44$ & 8.1 & Overall: 54.6 & $\approx 54$ & NR & NR & \begin{tabular}{|l|}
$\approx 26$ \\
$2-, 3-, 5-$ \\
$\mathrm{yr} 54.7 \%$, \\
$39.4 \%$ and \\
$18.4 \%$ \\
(two groups)
\end{tabular} & \begin{tabular}{|l}
$51.7(8.1$ \\
$89.3)$
\end{tabular} \\
\hline $\begin{array}{l}\text { Elias D/ 2007/ France } \\
{[36]}\end{array}$ & $\approx 96$ & $\approx 78$ & $\approx 63$ & $\approx 54$ & 54 & 0 & 4 & NA & NR & NR & NR & $\begin{array}{l}113 \\
188)\end{array}$ \\
\hline $\begin{array}{l}\text { Elias D/2009/ France } \\
\text { [28] }\end{array}$ & NR & 81 & NR & NR & 51 & NR & NR & 62.7 & NR & NR & NR & $\begin{array}{l}95.7 \text { vs } \\
63\end{array}$ \\
\hline $\begin{array}{l}\text { Elias D/ 2010/ France } \\
{[14]}\end{array}$ & NR & NR & 40 & NR & 25.5 & NR & NR & 31 & NR & NR & \begin{tabular}{|l}
$\approx 9$ \\
$1-, 3-, 5-\mathrm{yr}$ \\
$47 \%, \quad 15 \%$ \\
and $10 \%$ \\
(two groups)
\end{tabular} & NR \\
\hline $\begin{array}{lrr}\text { Esquivel J/ } & \text { 2014/ } \\
\text { America [29] } & \\
\end{array}$ & NR & NR & 66 & NR & 58 & NR & NR & 41 & $38.0-46.3$ & NR & NR & $\begin{array}{l}25 \text { vs. } 8 \\
(0.1-128)\end{array}$ \\
\hline $\begin{array}{l}\text { Franko J/ } \\
\text { America [37] }\end{array}$ & $\approx 92$ & $\approx 66$ & $\approx 51$ & $\approx 44$ & $\approx 28$ & NR & NR & 34.7 & NR & NR & NR & NR \\
\hline $\begin{array}{l}\text { Gervais MK/ 2013/ } \\
\text { Canada [38] }\end{array}$ & $\approx 92$ & $\approx 76$ & 61 & $\approx 53$ & 36 & 4 & 20 & $\approx 54$ & NR & NR & $\approx 8$ & $\begin{array}{l}22.8 \quad(2- \\
81)\end{array}$ \\
\hline $\begin{array}{l}\text { Glehen O/ 2004/ France } \\
{[13]}\end{array}$ & NR & NR & NR & NR & NR & NR & NR & $21.6 / 19.2$ & NR & NR & NR & $\begin{array}{l}53 \\
192)\end{array}$ \\
\hline $\begin{array}{l}\text { Goéré D/2015/ France } \\
\text { [99] }\end{array}$ & $\approx 90$ & $\approx 72$ & 52 & $\approx 40$ & $\approx 32$ & 5.8 & 29.5 & $\approx 35$ & NR & NR & NR & $\begin{array}{l}60 \\
74)\end{array}$ \\
\hline $\begin{array}{l}\text { Huang CQ/ 2014/ } \\
\text { China [39] }\end{array}$ & 63.6 & 20.0 & 16.0 & NR & NR & 0 & 28.6 & 13.7 & $10.0-16.5$ & NR & NR & \begin{tabular}{|l|}
41.5 \\
$(11.5-$ \\
$70.9)$ \\
\end{tabular} \\
\hline $\begin{array}{l}\text { Passot G/ 2014/ France } \\
{[40]}\end{array}$ & NR & NR & NR & NR & NR & NR & NR & 36 & NR & NR & NR & NR \\
\hline $\begin{array}{l}\text { Verwaal VJ/ 2003/ } \\
\text { Netherlands [12] }\end{array}$ & $\approx 66$ & $\approx 42$ & $\approx 32$ & NR & NR & 8 & 19 & 22.4 & NR & NR & NR & 21.6 \\
\hline $\begin{array}{l}\text { Subtotal of } 15 \text { studies } \\
\text { (Mean } \pm \text { SD; Median/ } \\
\text { Range) }\end{array}$ & $\begin{array}{l}84.5 \pm 12.6 \\
\text { vs. } \\
58.1 \pm 20.6 \\
91(63.6-96) \\
\text { vs. } \\
54(27.5-87)\end{array}$ & $\begin{array}{l}61.7 \pm 20.3 \\
\text { vs. } \\
38.8 \pm 18.7 \\
70(20-81) v s . \\
42(12-65)\end{array}$ & $\begin{array}{l}46.8 \pm 16.2 \\
v s . \\
23.6 \pm 15.2 \\
52 \quad(16-66) \\
v s . \\
18(0-47)\end{array}$ & $\begin{array}{l}48.8 \pm 6.4 v s . \\
20.4 \pm 10.1 \\
53(44-54) v s . \\
22(14-33)\end{array}$ & $\begin{array}{l}40.0 \pm 11.5 \\
v s . \\
18.1 \pm 14.1 \\
38(25.5-58) \\
v s . \\
18(0-44)\end{array}$ & $\begin{array}{l}4.3 \pm 3.7 \\
v s . \\
6.2 \pm 4.2 \\
5 \\
v s . \\
6.3 \quad(0-8.1) \\
11.1)\end{array}$ & $\begin{array}{l}19.8 \pm 9.2 v s . \\
20.5 \pm 12.3 \\
19.5(4-29.5) \\
v s . \\
23(3.1-31.6)\end{array}$ & $\begin{array}{l}34.3 \pm 14.8 \\
v s . \\
18.8 \pm 8.8 \\
35 .(13-62.7) \\
v s . \\
17(8.5-34)\end{array}$ & & & & \begin{tabular}{|l}
$43.8 \quad \pm$ \\
32.8 vs. \\
$29.7 \quad \pm$ \\
29.3 \\
$25 \quad(17$ \\
$113)$ vs. \\
$18(8-63)$
\end{tabular} \\
\hline
\end{tabular}

Note: yr: year; SR: survival rate; mo: months; OS: overall survival; PFS: progression-free survival; RFS: recurrence-free survival; DFS: disease-free survival; NA: not achieved; NR: not reported; PMP: pseudomyxoma peritonei; L-OHP: oxaliplatin; MMC: mitomycin; all: all tumors in researches; MVR: multivisceral resection group; NVR: No visceral resection group; APP: appendix; NNT: non-neoadjuvant therapy; NCA: neoadjuvant chemotherapy alone; NCB: neoadjuvant chemotherapy + bevacizumab; AC: adjuvant chemotherapy; NAC: non- adjuvant chemotherapy

included; (4) The key outcome measures should be included in literatures, such as OS, disease-free survival (DFS), recurrence-free survival (RFS), progression-free survival (PFS), year survival rate, morbidity and mortality [107], multivariate analysis, follow-up times; (5) English language; (6) To reduce the effect of publication bias, both fully published articles and abstracts were eligible for inclusion.

Exclusion criteria: (1) Animal studies, pathological research, imageology research, pharmacokinetics research, quality of life assessment, literature review, commentary, letter, book, etc; (2) Duplicate publication or overlapping data (chose the largest and latest sample size); (3) The sample size is less than 10; (4) Multiple cancer; (5) Unresectable liver metastases or others distant metastasis; (6) missing rate of follow-up $>5 \%$.

\section{Data extraction}

Three authors analyzed data from a meta-analysis of 15 controlled researches of CRS plus HIPEC group $v s$. surgery and/or SC group and a summarized analysis of 76 researches of HIPEC group. The following data were extracted from each article: (1) Major clinicopathologic characteristics and detail HIPEC regimens; (2) Survival and advent events. All relevant text, tables, and figures were reviewed for data extraction. For equivocal literatures or discrepancies between two independently assessed reviewers, these were resolved by discussion and consensus with a third author. 
Table 21: Survival of Patients with CRC PC Treated by CRS and HIPEC and/or EPIC and/or SC: Summary of 76 Researches.

\begin{tabular}{|c|c|c|c|c|c|c|c|c|c|c|c|c|}
\hline Author/Years/ Country & $\begin{array}{l}1-\mathrm{yr} \\
(\%)\end{array}$ & $\begin{array}{ll}2-\mathrm{yr} & \text { SR } \\
(\%) & \end{array}$ & $\begin{array}{ll}3-\mathrm{yr} & \text { SR } \\
(\%) & \end{array}$ & 4-yr SR (\%) & 5-yr SR (\%) & $\begin{array}{l}\text { Mortality } \\
\text { Rate }(\%)\end{array}$ & $\begin{array}{l}\text { Morbidity } \\
\text { Rate (\%) }\end{array}$ & $\begin{array}{l}\text { Median OS } \\
\text { (mo) }\end{array}$ & $\begin{array}{l}\text { OS 95\% CI } \\
\text { (mo) }\end{array}$ & $\begin{array}{l}\text { PFS }(95 \% \\
\text { CI) (mo) }\end{array}$ & \begin{tabular}{|l} 
DFS/RFS \\
(95\% CI) \\
(mo)
\end{tabular} & $\begin{array}{l}\text { Follow-up } \\
\text { times } \\
\text { (range) } \\
\text { (mo) }\end{array}$ \\
\hline \multicolumn{13}{|l|}{ HIPEC single arm studies } \\
\hline $\begin{array}{l}\begin{array}{l}\text { Alzahrani/ 2015/ Australia } \\
\text { [41] }\end{array} \\
\end{array}$ & $\approx 84$ & 56 & $\approx 40$ & $\approx 26$ & 24 & 1.2 & 23.3 & 28 & NR & NR & NR & NR \\
\hline Beaujard/ 2000/ France [42] & NR & NR & NR & NR & NR & NR & NR & 12 & NR & NR & NR & NR \\
\hline Bijelic/ 2008/ Australia [43] & $\approx 94$ & $\approx 56$ & $\approx 44$ & $\approx 23$ & 17 & NR & NR & 30 & NR & 15 & NR & \begin{tabular}{|l} 
Mean: 40.8 \\
Median: \\
29.5 \\
\end{tabular} \\
\hline Braam/2014/ Australia [44] & NR & NR & NR & $\mathrm{NR}$ & 6 & NR & NR & 14.9 & NR & NR & 11.4 & 26.2 \\
\hline $\mathrm{Cao} / 2009 /$ Australia [45] & 83.6 & 65.4 & 51.4 & 32.1 & 32.1 & NR & NR & 37.0 & $1-72$ & NR & NR & $19(1-72)$ \\
\hline Cavaliere/ 2006/ Italy [46] & NR & NR & 25.8 & NR & NR & 3.3 & 22.5 & 19 & NR & NR & 16 & 16 \\
\hline Ceelen/ 2014/ Belgium [47] & $\begin{array}{l}\approx 75 \text { (NNT) } \\
\approx 75 \text { (NCA) } \\
\approx 96 \text { (NCB) }\end{array}$ & $\begin{array}{l}\approx 57 \text { (NNT) } \\
\approx 47 \text { (NCA) } \\
\approx 89 \text { (NCB) }\end{array}$ & \begin{tabular}{|l}
$\approx 39$ (NNT) \\
$\approx 30$ (NCA) \\
$\approx 71$ (NCB)
\end{tabular} & $\begin{array}{l}\approx 32 \text { (NNT) } \\
\approx 19 \text { (NCA) } \\
\text { NA (NCB) }\end{array}$ & $\begin{array}{l}\approx 25(\mathrm{NNT}) \\
\approx 13(\mathrm{NCA})\end{array}$ & NR & NR & \begin{tabular}{|l} 
\\
27 \\
27 \\
(included \\
APP) \\
24 (Right \\
colon) \\
27 (Left \\
colon) \\
35 (Rectal) \\
25 (NNT) \\
22 (NCA) \\
39 (NCB) \\
30 (AC) \\
22 (NAC)
\end{tabular} & \begin{tabular}{|l|}
$20.8-33.2$ \\
(included \\
APP) \\
$10.3-37.7$ \\
(Right \\
colon) \\
$22.8-31.2$ \\
(Left colon) \\
$4.9-65$ \\
(Rectum) \\
$19.1-30.9$ \\
(NNT) \\
$12.9-31.1$ \\
(NCA) \\
$17.6-60.4$ \\
(NCB) \\
$20.7-39.3$ \\
(AC) \\
$14.2-29.8$ \\
(NAC) \\
\end{tabular} & NR & NR & 18 \\
\hline
\end{tabular}

Note: yr: year; SR: survival rate; mo: months; OS: overall survival; PFS: progression-free survival; RFS: recurrence-free survival; DFS: disease-free survival; NA: not achieved; NR: not reported; PMP: pseudomyxoma peritonei; L-OHP: oxaliplatin; MMC: mitomycin; all: all tumors in researches; MVR: multivisceral resection group; NVR: No visceral resection group; APP: appendix; NNT: non-neoadjuvant therapy; NCA: neoadjuvant chemotherapy alone; NCB: neoadjuvant chemotherapy + bevacizumab; AC: adjuvant chemotherapy; NAC: non- adjuvant chemotherapy

Table 22: Survival of Patients with CRC PC Treated by CRS and HIPEC and/or EPIC and/or SC: Summary of 76 Researches.

\begin{tabular}{|c|c|c|c|c|c|c|c|c|c|c|c|c|}
\hline $\begin{array}{ll}\text { Author/ } & \text { Years/ } \\
\text { Country } & \end{array}$ & $\begin{array}{ll}1-\mathrm{yr} & \mathrm{SR} \\
(\%) & \end{array}$ & 2-yr SR (\%) & 3-yr SR (\%) & $\begin{array}{ll}4-y r & \text { SR } \\
(\%) & \end{array}$ & $\begin{array}{ll}5-y r & \text { SR } \\
(\%) & \end{array}$ & $\begin{array}{l}\text { Mortality } \\
\text { Rate (\%) }\end{array}$ & $\begin{array}{l}\text { Morbidity } \\
\text { Rate (\%) }\end{array}$ & $\begin{array}{l}\text { Median OS } \\
\text { (mo) }\end{array}$ & $\begin{array}{l}\text { OS 95\% CI } \\
\text { (mo) }\end{array}$ & $\begin{array}{l}\text { PFS }(95 \% \\
\text { CI) (mo) }\end{array}$ & $\begin{array}{l}\text { DFS/RFS } \\
(95 \% \text { CI) (mo) }\end{array}$ & \begin{tabular}{|l|} 
Follow-up \\
times \\
(range) (mo)
\end{tabular} \\
\hline \multicolumn{13}{|l|}{ HIPEC single arm studies } \\
\hline $\begin{array}{l}\text { Desantis/2014/France } \\
\text { [48] }\end{array}$ & $\approx 88$ & $\approx 72$ & 60.3 & $\approx 47$ & 37 & 1 (all) & 12.5 (all) & 45.9 & NR & NR & \begin{tabular}{|l}
16.8 \\
$1-, 3-, 5-\mathrm{yr} \quad 61.3 \%$, \\
$30.4 \%$ and $22.8 \%$
\end{tabular} & NR \\
\hline $\begin{array}{lll}\text { Elias/ 2004/ France } \\
{[49]} & & \\
\end{array}$ & 83 & 74 & 65 & NR & NR & 8.3 & 41.6 & NR & NR & NR & $\begin{array}{l}18 \\
1-, 2-, 3-\mathrm{yr} \quad 61 \% \text {, } \\
50 \% \text { and } 50 \%\end{array}$ & $\begin{array}{l}27.4 \\
49.6)\end{array}$ \\
\hline $\begin{array}{lll}\text { Elias/ 2014/ } & \text { France } \\
{[50]} & & \\
\end{array}$ & 91.4 & $\approx 74$ & 54 & $\approx 47$ & 36.5 & 4.2 & 17 & $\approx 41$ & NR & NR & NR & $\begin{array}{l}62.4 \quad(55.6- \\
77.6)\end{array}$ \\
\hline $\begin{array}{l}\text { Evers/ } \\
\text { Netherlands [51] }\end{array}$ & NR & NR & NR & NR & 36 & NR & NR & $\begin{array}{l}49.2 \text { vs. } 41.3 \\
\text { (Ovarian } \\
\text { metastases } \\
\text { vs. without } \\
\text { ovarian } \\
\text { metastases) }\end{array}$ & NR & NR & $\begin{array}{l}36.9 \text { vs. } 32.5 \\
\text { (Ovarian metastases } \\
\text { vs. without ovarian } \\
\text { metastases) }\end{array}$ & $\begin{array}{l}22(1 \text { week - } \\
97 \text { mo) }\end{array}$ \\
\hline 100] & NR & NR & NR & NR & 42 & 4.6 & 47 & 41 & $32-50$ & NR & $\begin{array}{l}17.7(12-19) \\
5 \text {-yr: } 14 \%\end{array}$ & $\begin{array}{ll}48.5 & (41.0- \\
56.3) & \\
\end{array}$ \\
\hline $\begin{array}{l}\text { Franko/ 2008/ America } \\
\text { [52] }\end{array}$ & $\begin{array}{l}\approx 79 \\
(\mathrm{MVR}) \\
\approx 12(\mathrm{NVR})\end{array}$ & $\begin{array}{l}\approx 46 \text { (MVR) } \\
\approx 30 \text { (NVR) }\end{array}$ & $\begin{array}{l}\approx 31 \text { (MVR) } \\
\approx 30 \text { (NVR) }\end{array}$ & $\begin{array}{l}\approx 16 \\
\text { (MVR) } \\
\approx 30 \\
\text { (NVR) } \\
\end{array}$ & $\begin{array}{l}0 \\
\text { (MVR) } \\
\approx 15 \\
\text { (NVR) } \\
\end{array}$ & 1.4 & 60 & $\begin{array}{l}20.2 \text { (MVR) } \\
14.3 \text { (NVR) }\end{array}$ & NR & NR & NR & NR \\
\hline $\begin{array}{ll}\text { Frøysnes/ } & 2016 / \\
\text { Norway [103] } & \\
\end{array}$ & $\approx 93$ & $\approx 78$ & 65 & $\approx 45$ & 36 & 0 & 15.1 & 47 & $42-52$ & NR & $10(7-12)$ & $45(35-55)$ \\
\hline $\begin{array}{l}\text { Glehen/ 2003/ France } \\
\text { [53] }\end{array}$ & NR & NR & NR & NR & NR & 1.8 (all) & 28.6 (all) & 17.5 & $4.4-53.6$ & NR & NR & $\begin{array}{l}18.1(4.4-56) \\
\text { (all) }\end{array}$ \\
\hline $\begin{array}{l}\text { Glehen/ 2004/ France } \\
\text { [16] }\end{array}$ & 55 & 32 & NR & NR & 11 & 4 & 23 & 12.8 & NR & NR & NR & $59.5(2-148)$ \\
\hline $\begin{array}{l}\text { Glehen/ 2010/ France } \\
\text { [54] }\end{array}$ & $\approx 80$ & $\approx 56$ & 41 & $\approx 33$ & 26 & 4.1 (all) & 33.6 (all) & 30 & NR & NR & $\begin{array}{l}1-, 3-, 5-\mathrm{yr} \\
49 \% \text { and } 37 \%\end{array}$ & $\begin{array}{l}45.3 \text { (20.3- } \\
90.9) \text { (all) }\end{array}$ \\
\hline \begin{tabular}{lcc|} 
Gomes & $/$ & $2005 /$ \\
America [55] & \\
\end{tabular} & $\approx 60$ & $\approx 30$ & $\approx 20$ & $\approx 20$ & 0 & NR & NR & 20 & NR & NR & NR & $15.7(1-51)$ \\
\hline $\begin{array}{l}\text { Gusani/2008/ America } \\
{[56]}\end{array}$ & $\approx 74$ & $\approx 49$ & $\approx 49$ & $\approx 39$ & NR & 0 & 29.8 (all) & $\approx 23.6$ & NR & NR & NR & $\begin{array}{l}35.9 \text { (19.0- } \\
57.7) \text { (all) }\end{array}$ \\
\hline $\begin{array}{ll}\text { Hamilton/ } & \text { 2011/ } \\
\text { Canada [57] } & \\
\end{array}$ & $\approx 79$ & $\approx 62$ & 38 & $\approx 34$ & 34 & NR & NR & 27 & $0-87$ & NR & $\begin{array}{l}9(0-87) \\
3-, 5-\mathrm{yr} 34 \%, 26 \%\end{array}$ & \begin{tabular}{|ll}
28 & $(0-119)$ \\
(all)
\end{tabular} \\
\hline $\begin{array}{ll}\text { Hompes/ } & \text { 2012/ } \\
\text { Belgium [58] } & \end{array}$ & 97.9 & 88.7 & $\approx 84$ & NA & NA & 0 & 52.1 & NA & NA & NR & $\begin{array}{l}19.8 \text { (12-upper } \\
\text { limit not defined) } \\
\text { (RFS) }\end{array}$ & $\begin{array}{l}22.7 \\
55.7)\end{array}$ \\
\hline
\end{tabular}

Note: yr: year; SR: survival rate; mo: months; OS: overall survival; PFS: progression-free survival; RFS: recurrence-free survival; DFS: disease-free survival; NA: not achieved; NR: not reported; PMP: pseudomyxoma peritonei; L-OHP: oxaliplatin; MMC: mitomycin; all: all tumors in researches; MVR: multivisceral resection group; NVR: No visceral resection group; APP: appendix; NNT: non-neoadjuvan therapy; NCA: neoadjuvant chemotherapy alone; NCB: neoadjuvant chemotherapy + bevacizumab; AC: adjuvant chemotherapy; NAC: non- adjuvant chemotherapy 
Table 23: Survival of Patients with CRC PC Treated by CRS and HIPEC and/or EPIC and/or SC: Summary of 76 Researches.

\begin{tabular}{|c|c|c|c|c|c|c|c|c|c|c|c|c|}
\hline Author/ Years/ Country & 1-yr SR (\%) & 2-yr SR (\%) & 3-yr SR (\%) & 4-yr SR (\%) & $\begin{array}{l}5 \text {-yr } \\
\text { SR } \\
(\%)\end{array}$ & $\begin{array}{l}\text { Mortality } \\
\text { Rate (\%) }\end{array}$ & $\begin{array}{l}\text { Morbidity } \\
\text { Rate (\%) }\end{array}$ & $\begin{array}{l}\text { Median OS } \\
\text { (mo) }\end{array}$ & $\begin{array}{l}\text { OS } 95 \% \\
\text { CI } \\
\text { (mo) }\end{array}$ & $\begin{array}{l}\text { PFS }(95 \% \\
\text { CI) (mo) }\end{array}$ & $\begin{array}{l}\text { DFS/RFS } \\
(95 \% \text { CI) (mo) }\end{array}$ & $\begin{array}{l}\text { Follow- } \\
\text { up times } \\
\text { (range) } \\
\text { (mo) }\end{array}$ \\
\hline \multicolumn{13}{|l|}{ HIPEC single arm studies } \\
\hline $\begin{array}{l}\text { Hompes/ 2014/ Belgium } \\
{[59]}\end{array}$ & $\begin{array}{l}\approx 91 \text { (L-OHP) } \\
\approx 88 \text { (MMC) }\end{array}$ & $\begin{array}{l}\approx 68 \text { (L-OHP) } \\
\approx 59 \text { (MMC) }\end{array}$ & $\begin{array}{l}\approx 53 \text { (L-OHP) } \\
\approx 42(\mathrm{MMC})\end{array}$ & $\begin{array}{l}\approx 45 \text { (L-OHP) } \\
\approx 33 \text { (MMC) }\end{array}$ & NA & 0 & 41.1 & $\begin{array}{l}37.1 \text { (L-OHP) } \\
26.5 \text { (MMC) }\end{array}$ & $\begin{array}{l}22.4- \\
52.8 \\
\text { (L-OHP) } \\
16.9- \\
64.8 \\
(\mathrm{MMC})\end{array}$ & NR & $\begin{array}{l}12.2 \\
\text { (7.2-undefined) } \\
\text { (L-OHP) } \\
13.8(7.0-25.8) \\
\text { (MMC) (RFS) }\end{array}$ & $\begin{array}{l}33.6 \\
\text { (L-OHP) } \\
61.2 \\
\text { (MMC) }\end{array}$ \\
\hline $\begin{array}{l}\text { Iversen/ 2013/ Denmark } \\
{[60]}\end{array}$ & $\approx 97$ & 60 & 47 & 38 & 38 & 2.9 & 32.4 & $\approx 31$ & NR & NR & NR & $\begin{array}{l}16.0 \\
(0.9- \\
71.3)\end{array}$ \\
\hline $\begin{array}{l}\text { Kecmanovic/ 2005/ Serbia } \\
\text { and Montenegro [61] }\end{array}$ & $\approx 85$ & $\approx 85$ & $\approx 85$ & $\approx 85$ & NA & 0 & 44.4 & 15 & $1-57$ & NR & NR & $\begin{array}{ll}21 \\
56)\end{array}$ \\
\hline $\begin{array}{l}\text { Kianmanesh/ 2007/ France } \\
\text { [62] }\end{array}$ & $\approx 95$ & 72 & $\approx 57$ & 44 & 44 & 2.3 & 39 & 38.4 & $\begin{array}{l}32.8- \\
43.9\end{array}$ & NR & NR & NR \\
\hline $\begin{array}{l}\text { Klaver/2011/ Netherlands } \\
\text { [63] }\end{array}$ & 71 & $\approx 56$ & $\approx 43$ & $\approx 35$ & $\approx 18$ & NR & NR & 28 & $3-100$ & NR & NR & NR \\
\hline $\begin{array}{l}\text { Klaver/2012/ Netherlands } \\
\text { [64] }\end{array}$ & 83 & $\approx 52$ & $\approx 26$ & $\approx 26$ & NA & 0 & 33.3 & 35 & $\begin{array}{l}20.0- \\
49.9\end{array}$ & NR & $12(7.7-16.3)$ & $\begin{array}{l}10.5)(1- \\
52)\end{array}$ \\
\hline $\begin{array}{l}\text { Kuijpers/ 2013/ Netherlands } \\
{[65]}\end{array}$ & $\approx 84$ & $\approx 62$ & 46 & $\approx 37$ & 31 & $\begin{array}{l}3 \\
\text { included } \\
\text { PMP }\end{array}$ & $\begin{array}{l}34 \\
\text { included } \\
\text { PMP }\end{array}$ & 33 & $28-38$ & $15(13-17)$ & NR & $\begin{array}{l}41 \quad(35- \\
46) \\
\text { included } \\
\text { PMP } \\
\end{array}$ \\
\hline $\begin{array}{l}\text { Kuijpers/2014/ Netherlands } \\
{[66]}\end{array}$ & $\approx 87$ & $\approx 62$ & 45 & $\approx 37$ & $\approx 32$ & 0 & 30 & 30 & $19-41$ & $15(14-16)$ & NR & $\begin{array}{l}47 \quad(43- \\
51)\end{array}$ \\
\hline Lanuke/ 2009/ Canada [67] & $\approx 85$ & $\approx 58$ & $\approx 46$ & NA & NA & 4 (all) & 39 (all) & 26 & $1-48$ & NR & $8(1-31)$ & $\begin{array}{ll}12 \\
48)\end{array}$ \\
\hline Levine/ 2014/ America [68] & $\approx 69$ & $\approx 38$ & $\approx 27$ & $\approx 19$ & $\approx 17$ & 3.8 (all) & 42 (all) & $\approx 19$ & NR & NR & NR & NR \\
\hline Maillet/2016/ France [101] & NR & NR & 58 & NR & 34 & 4 & NR & 43.3 & NR & 12.4 & NR & NR \\
\hline $\begin{array}{l}\text { McConnell/ 2013/ Canada } \\
\text { [69] }\end{array}$ & NR & NR & NR & NR & NR & 0 & 36.9 & NR & NR & NR & NR & NR \\
\hline Nikolic/ 2014/ Serbia [70] & 78.6 & 58.7 & $\approx 53$ & $\approx 50$ & $\approx 42$ & NR & NR & 51 & $>22$ & NR & $\begin{array}{l}23(>16) \\
1-, 2-, 6-\mathrm{yr} \\
68.3 \%, 46.7 \% \\
\text { and } 38.1 \% \\
\end{array}$ & $\begin{array}{l}22 \\
83)\end{array}$ \\
\hline Passot/2012/ France [21] & 77 & 51 & NR & NR & 33 & NR & NR & 36.2 & NR & NR & NR & $\begin{array}{l}58.5 \\
183)\end{array}$ \\
\hline Passot/ 2016/ France [104] & $\approx 83$ & $\approx 65$ & $\approx 51$ & $\approx 38$ & 31 & NR & 30 & 36 & NR & NR & 11 & NR \\
\hline Pilati/ 2003/ Italy [71] & $\approx 68$ & 31 & NR & NR & NR & 0 & 35 & 18 & NR & 13 & NR & 14.5 \\
\hline $\begin{array}{l}\text { Prada-Villaverde/ 2014/ } \\
\text { Spain [72] }\end{array}$ & $\approx 85$ & $\approx 63$ & $\approx 45$ & $\approx 38$ & $\approx 35$ & NR & NR & 31.4 & NR & NR & NR & NR \\
\hline Quenet/ 2011/ France [73] & $\approx 92$ & $\approx 72$ & $\approx 36$ & $\approx 47$ & $\approx 44$ & 4.1 & 47.2 & 41 & $32-60$ & NR & $\begin{array}{l}15.7 \\
\text { (RFS) }\end{array}$ & $\begin{array}{l}48.5 \\
(41.0- \\
56.3)\end{array}$ \\
\hline Rivard/2014/Canada [74] & $\begin{array}{l}\approx 88 \text { (Colon) } \\
\approx 80 \text { (Rectal) }\end{array}$ & $\begin{array}{l}\approx 68 \text { (Colon) } \\
\approx 24 \text { (Rectal) }\end{array}$ & $\begin{array}{l}\approx 46 \text { (Colon) } \\
\approx 30 \text { (Rectal) }\end{array}$ & NA & NA & NR & NR & $\begin{array}{l}\approx 31 \text { (Colon) } \\
\approx 18 \text { (Rectal) }\end{array}$ & NR & NR & $\begin{array}{l}10.9 \\
3-y r, 15 \%\end{array}$ & $\begin{array}{l}30.3 \quad(2- \\
88)\end{array}$ \\
\hline
\end{tabular}

Note: yr: year; SR: survival rate; mo: months; OS: overall survival; PFS: progression-free survival; RFS: recurrence-free survival; DFS: disease-free survival; NA: not achieved; NR: not reported; PMP: pseudomyxoma peritonei; L-OHP: oxaliplatin; MMC: mitomycin; all: all tumors in researches; MVR: multivisceral resection group; NVR: No visceral resection group; APP: appendix; NNT: non-neoadjuvant therapy; NCA: neoadjuvant chemotherapy alone; NCB: neoadjuvant chemotherapy + bevacizumab; AC: adjuvant chemotherapy; NAC: non- adjuvant chemotherapy

\section{Statistical methods}

All meta-analysis were performed using Review Manager 5. Overall survival (OS) or disease-free survival (DFS) in all studies were extracted from original literature. If not achieved accurate data in original text, hazard ratios (HRs) for time-to-event outcomes with $95 \%$ confidence intervals $(95 \% \mathrm{CI})$ in two groups were estimated by Tierney's methods [108]. The heterogeneity in the metaanalysis was evaluated by $I^{2}$ statistics [109] and $T$ test [110] was calculated for each result in summarizing analysis of all HIPEC-related data from the included 76 articles. If $I^{2}>50 \%$, it was defined as the unacceptable heterogeneity. If $I^{2}<50 \%$, fixed effect model was used to get pooled $\mathrm{HR}$ and $95 \% \mathrm{CI}$; otherwise, random effects model was used if moderate heterogeneity. For a sensitivity analysis, we investigated the different research features of eligible trials, which included statistical methods, methodological quality, sample sizes, and clinical factors on HIPEC-related effect, after that, summarizing each subgroup data in term of Mental-Haenszel stratification analysis. According to Egger's test [111] and Begg's test [112], publication bias was considered to be inevitable when $P<0.10$. The funnel plot analyses using 'STATA: Data Analysis and Statistical Software version 12.0', was to observe the results of meta-analysis whether any publication bias. 
Table 24: Survival of Patients with CRC PC Treated by CRS and HIPEC and/or EPIC and/or SC: Summary of 76 Researches.

\begin{tabular}{|c|c|c|c|c|c|c|c|c|c|c|c|c|}
\hline \begin{tabular}{ll|l}
$\begin{array}{l}\text { Author/ } \\
\text { Country }\end{array}$ & Years/
\end{tabular} & 1-yr SR (\%) & 2-yr SR (\%) & $\begin{array}{l}\begin{array}{l}3-\mathrm{yr} \\
(\%)\end{array} \\
\end{array}$ & $\begin{array}{ll}\begin{array}{l}\text { 4-yr } \\
(\%)\end{array} & \text { SR } \\
\end{array}$ & $\begin{array}{ll}5-y r & \text { SR } \\
(\%) & \end{array}$ & $\begin{array}{l}\text { Mortality } \\
\text { Rate (\%) }\end{array}$ & $\begin{array}{l}\text { Morbidity } \\
\text { Rate (\%) }\end{array}$ & $\begin{array}{l}\text { Median OS } \\
\text { (mo) }\end{array}$ & $\begin{array}{l}\text { OS 95\% CI } \\
\text { (mo) }\end{array}$ & $\begin{array}{l}\text { PFS }(95 \% \\
\text { CI) (mo) }\end{array}$ & $\begin{array}{l}\text { DFS/RFS } \\
(95 \% \text { CI) (mo) }\end{array}$ & \begin{tabular}{|l} 
Follow-up \\
times \\
(range) (mo)
\end{tabular} \\
\hline \multicolumn{13}{|l|}{ HIPEC single arm studies } \\
\hline $\begin{array}{l}\text { Rodt/ 2013/ Denmark } \\
{[75]}\end{array}$ & \begin{tabular}{l|l}
$\mathrm{k}$ & $\approx 52$ \\
\end{tabular} & $\approx 36$ & $\approx 12$ & 0 & 0 & 0 & 9.4 (all) & 12.7 & $4.0-21.4$ & NR & NR & $13(1-44)$ \\
\hline $\begin{array}{l}\text { Shen/ 2004/ America } \\
{[20]}\end{array}$ & \begin{tabular}{|l|l|} 
& $N R$ \\
\end{tabular} & NR & 25 & NR & 17 & 12 & 30 & 16 & $10-26$ & $7(3-31)$ & NR & 15 \\
\hline $\begin{array}{l}\text { Shen/ 2008/ America } \\
{[76]}\end{array}$ & 91 & $\approx 60$ & 48 & $\approx 32$ & 26 & 5.5 & 41.8 & 34 & $23-45$ & NR & NR & 86 \\
\hline $\begin{array}{l}\text { Simkens/ 2015/ } \\
\text { Netherlands [102] }\end{array}$ & NR & NR & 42 & NR & NR & 3 & 24.8 & 27 & $18.8-35.3$ & NR & 1-yr: $35 \%$ & $22.9(0.4-75.3)$ \\
\hline $\begin{array}{l}\text { Swellengrebel/ 2009/ } \\
\text { Netherlands [77] }\end{array}$ & $\mathrm{NR}$ & NR & NR & NR & NR & NR & NR & 25.6 & 20.9-29.4 & $\begin{array}{l}13.6(11.2- \\
16.4)\end{array}$ & NR & NR \\
\hline $\begin{array}{l}\text { Tabrizian/ } \\
\text { America [78] }\end{array}$ & 74.0 & $\approx 47$ & 89.4 & NA & NA & NR & NR & NR & NR & NR & $\begin{array}{l}12.4 \pm 1.8 \text { (RFS) } \\
1-3-\mathrm{yr}, \\
46.9 \%, 73.9 \% \\
\end{array}$ & $15.7 \pm 1.2$ \\
\hline $\begin{array}{l}\text { Teo/ 2013/ Singapore } \\
\text { [79] }\end{array}$ & $\approx 87$ & $\approx 58$ & $\approx 36$ & $\approx 18$ & $\approx 18$ & 0 & 56 (all) & $\approx 28$ & NR & NR & $\approx 10$ & $\begin{array}{l}21 \\
\text { (all) }\end{array}$ \\
\hline $\begin{array}{l}\text { Teo/ 2014/ Singapore } \\
{[80]}\end{array}$ & 83.7 & $\approx 53$ & 38.2 & 19.1 & 19.1 & 0 & 40 & 27.1 & $15.3-39.1$ & NR & $\begin{array}{l}9.4(5.5-18.7) \\
1-, 3-, 5-\mathrm{yr}, \\
43.8 \%, 22.3 \% \text {, } \\
22.3 \%\end{array}$ & $24.7(0.6-81.8)$ \\
\hline $\begin{array}{l}\text { Ung/ 2013/ Australia } \\
\text { [81] }\end{array}$ & $\approx 84$ (Colon) & $\approx 63$ (Colon) & $\begin{array}{l}\approx 53 \\
\text { (Colon) }\end{array}$ & $\begin{array}{l}\approx 37 \\
(\text { Colon })\end{array}$ & $\begin{array}{l}33 \\
\text { (Colon) }\end{array}$ & NR & NR & \begin{tabular}{|l|}
37.1 (Colon) \\
29.6 (Rectal)
\end{tabular} & NR & NR & $\begin{array}{l}12.6 \text { (Colon) } \\
19.0 \text { (Rectal) }\end{array}$ & $\begin{array}{ll}\begin{array}{l}23.3 \\
\text { (all) }\end{array} & (1-156) \\
\end{array}$ \\
\hline Vaira/ 2010/ Italy [82] & \begin{tabular}{|l}
100 \\
(L-OHP) \\
$\approx 61(\mathrm{MMC})$
\end{tabular} & $\begin{array}{l}\text { ₹60 } \\
\text { (L-OHP) } \\
\approx 17 \text { (MMC) }\end{array}$ & $\begin{array}{l}\approx 18 \\
\text { (L-OHP) } \\
\approx 9 \text { (MMC) }\end{array}$ & $\begin{array}{l}\approx 18 \\
(\mathrm{~L}-\mathrm{OHP}) \\
\approx 4 \\
(\mathrm{MMC})\end{array}$ & $\begin{array}{l}\text { NA } \\
\text { (L-OHP) } \\
0 \\
(\mathrm{MMC})\end{array}$ & 2.5 & 55 & $\begin{array}{l}24.6 \text { (L-OHP) } \\
16.6 \text { (MMC) }\end{array}$ & NR & NR & NR & NR \\
\hline $\begin{array}{l}\text { van Leeuwen / 2008/ } \\
\text { Sweden 83] }\end{array}$ & $\approx 82$ & $\approx 65$ & NA & NA & NA & $>1$ (all) & 56.3 (all) & NA & NA & NR & 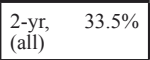 & $13(2-37)$ (all) \\
\hline van Oudheus84] & $\approx 86$ & $\approx 70$ & $\approx 43$ & $\approx 30$ & $\approx 22$ & 1.8 & 22.1 & 36.1 & NR & NR & NR & $16.2(0.13-90)$ \\
\hline $\begin{array}{lr}\text { van } & \text { Oudheusden / } \\
2015 / & \text { Netherlands } \\
{[85]} & \end{array}$ & $\approx 87$ & $\approx 68$ & 44 & $\approx 38$ & $\approx 27$ & NR & 13.5 & 35.1 & NR & NR & NR & $12.7(0.10-90.2)$ \\
\hline $\begin{array}{l}\text { Varban/2009/America } \\
{[86]}\end{array}$ & $\approx 63$ & 36.8 & $\approx 25$ & 17.4 & $\approx 16$ & 7.7 & 40.1 & 15.8 & $13.5-20.2$ & NR & NR & 13.4 \\
\hline $\begin{array}{l}\text { Verwaal/ 2005/ } \\
\text { Netherlands [19] }\end{array}$ & 75 & NR & 28 & NR & 19 & NR & NR & 21.8 & $19.0-25.5$ & NR & NR & 46 \\
\hline $\begin{array}{ll}\text { Votanopoulos/ } & \text { 2013/ } \\
\text { America [87] } & \end{array}$ & $\begin{array}{l}\approx 63 \text { (Colon) } \\
\approx 83 \\
(\text { Rectal })\end{array}$ & $\begin{array}{l}\approx 31 \text { (Colon) } \\
\approx 36 \text { (Rectal) }\end{array}$ & $\begin{array}{l}25.1 \\
\text { (Colon) } \\
28.2 \\
\text { (Rectal) }\end{array}$ & NR & NR & $\begin{array}{l}5.7 \text { (Colon) } \\
0 \text { (Rectal) }\end{array}$ & $\begin{array}{l}57 \text { (Colon) } \\
46 \text { (Rectal) }\end{array}$ & $\begin{array}{l}17.3 \text { (Colon) } \\
14.6 \text { (Rectal) }\end{array}$ & NR & NR & NR & $\begin{array}{l}88.1 \text { (Colon) } \\
40.1 \text { (Rectal) }\end{array}$ \\
\hline
\end{tabular}

Note: yr: year; SR: survival rate; mo: months; OS: overall survival; PFS: progression-free survival; RFS: recurrence-free survival; DFS: disease-free survival; NA: not achieved; NR: not reported; PMP: pseudomyxoma peritonei; L-OHP: oxaliplatin; MMC: mitomycin; all: all tumors in researches; MVR: multivisceral resection group; NVR: No visceral resection group; APP: appendix; NNT: non-neoadjuvant therapy; NCA: neoadjuvant chemotherapy alone; NCB: neoadjuvant chemotherapy + bevacizumab; AC: adjuvant chemotherapy; NAC: non- adjuvant chemotherapy

Table 25: Survival of Patients with CRC PC Treated by CRS and HIPEC and/or EPIC and/or SC: Summary of 76 Researches.

\begin{tabular}{|c|c|c|c|c|c|c|c|c|c|c|c|c|}
\hline \begin{tabular}{ll|l} 
Author/ & Years $/$ \\
Country & & 1-
\end{tabular} & $\begin{array}{ll}1-\mathrm{yr} & \mathrm{SR} \\
(\%) & \end{array}$ & 2-yr SR (\%) & $\begin{array}{ll}\begin{array}{l}3-\mathrm{yr} \\
(\%)\end{array} & \text { SR } \\
\end{array}$ & $\begin{array}{l}\text { 4-yr SR } \\
(\%)\end{array}$ & $\begin{array}{l}\text { 5-yr } \\
\text { SR } \\
(\%)\end{array}$ & $\begin{array}{l}\text { Mortality } \\
\text { Rate (\%) }\end{array}$ & $\begin{array}{l}\text { Morbidity } \\
\text { Rate (\%) }\end{array}$ & $\begin{array}{l}\text { Median OS } \\
\text { (mo) }\end{array}$ & $\begin{array}{l}\text { OS } \\
95 \% \\
\text { CI } \\
\text { (mo) } \\
\end{array}$ & $\begin{array}{l}\text { PFS }(95 \% \\
\text { CI) (mo) }\end{array}$ & $\begin{array}{l}\text { DFS/RFS } \\
\left(\begin{array}{ll}95 \% \\
(\mathrm{mo})\end{array}\right.\end{array}$ & $\begin{array}{l}\begin{array}{l}\text { Follow- } \\
\text { up times } \\
\text { (range) } \\
\text { (mo) }\end{array} \\
\end{array}$ \\
\hline \multicolumn{13}{|l|}{ HIPEC single arm studies } \\
\hline $\begin{array}{l}\text { Winer/ 2014/ America } \\
{[88]}\end{array}$ & 53 & 22 & 22 & $\approx 13$ & $\approx 13$ & 6.7 & 22.2 & 12.2 & $\begin{array}{l}7.5- \\
17.2\end{array}$ & $\begin{array}{l}9.3 \quad(3.3- \\
17.8) \\
1-3-\mathrm{yr}, \\
47 \%, 16 \%\end{array}$ & NR & $\begin{array}{l}52.8 \\
(12.5- \\
138)\end{array}$ \\
\hline $\begin{array}{l}\text { Witkamp/ 2001/ } \\
\text { Netherlands [89] }\end{array}$ & NR & 45 & 23 & NR & NR & 3 & 38 & NR & NR & NR & $\begin{array}{l}11 \quad(3-29) \\
\text { (RFS) }\end{array}$ & \begin{tabular}{|lr}
38 & $(26-$ \\
$52)$ & \\
\end{tabular} \\
\hline $\begin{array}{l}\text { Yan/ 2006/ Australia } \\
{[90]}\end{array}$ & 72 & 64 & NR & NR & NR & 0 & NR & 29 & $2-39$ & NR & NR & $12(2-39)$ \\
\hline $\begin{array}{l}\text { Yan/ 2008/ Australia } \\
\text { [91] }\end{array}$ & 79 & 67 & 39 & NR & NR & NR & NR & 29 & $1-56$ & NR & NR & $14(1-56)$ \\
\hline Zanon/ 2006/Italy [92] & $\approx 75$ & $\approx 60$ & $\approx 28$ & NR & NR & 4 & 24 & 30.3 & $\begin{array}{l}17.0- \\
52.2\end{array}$ & $\begin{array}{l}17.3 \\
(5.72- \\
28.9)\end{array}$ & NR & NR \\
\hline $\begin{array}{l}\text { Total of } 76 \text { studies } \\
\text { (Mean } \pm \text { SD; Median/ } \\
\text { Range) }\end{array}$ & \begin{tabular}{|lr}
79.7 & \pm \\
$14.5 ;$ \\
83 \\
$100)$
\end{tabular} & $\begin{array}{l}56.5 \pm 17.3 \\
60(17-89)\end{array}$ & $\begin{array}{l}42.3 \pm 17.1 \\
42(9-89.4)\end{array}$ & $\begin{array}{l}33.8 \quad \pm \\
15.4 ; \\
34.5(0- \\
85)\end{array}$ & \begin{tabular}{|l|}
$27.5 \pm$ \\
$14.1 ;$ \\
$31(0-$ \\
$58)$
\end{tabular} & $\begin{array}{l}2.8 \pm 2.9 \\
2.5(0-12)\end{array}$ & $\begin{array}{l}33.0 \pm 13.4 \\
32.9(4-60)\end{array}$ & $\begin{array}{l}29.2 \pm 11.3 \\
29 \\
62.7)\end{array}$ & & $\begin{array}{l}13.1 \pm 3.2 \\
13.6 \\
17.3)\end{array}$ & $\begin{array}{l}15.9 \pm 7.7 \\
12.6 \\
36.9)\end{array}$ & $\begin{array}{ll}33.1 & \pm \\
22.5 & \end{array}$ \\
\hline
\end{tabular}

Note: yr: year; SR: survival rate; mo: months; OS: overall survival; PFS: progression-free survival; RFS: recurrence-free survival; DFS: disease-free survival; NA: not achieved; NR: not reported; PMP: pseudomyxoma peritonei; L-OHP: oxaliplatin; MMC: mitomycin; all: all tumors in researches; MVR: multivisceral resection group; NVR: No visceral resection group; APP: appendix; NNT: non-neoadjuvant therapy; NCA: neoadjuvant chemotherapy alone; NCB: neoadjuvant chemotherapy + bevacizumab; AC: adjuvant chemotherapy; NAC: non- adjuvant chemotherapy 


\section{ACKNOWLEDGEMENTS AND GRANT SUPPORT}

This study was supported by the grants for the Young Teacher Foundation of the Fundamental Research Fund for the Central Universities (No. 413000009), the Wuhan Clinical Research Center for Peritoneal Carcinomatosis (No. 2015060911020462), the Hubei Province's Outstanding Medical Academic Leader Program (No. [2013]4) and the Science Fund for Doctorate Mentors by China's Ministry of Education (No. 20120141110042).

\section{CONFLICTS OF INTEREST}

The authors declare that there are no conflicts of interest.

\section{REFERENCES}

1. Chu DZ, Lang NP, Thompson C, Osteen PK, Westbrook KC. Peritoneal carcinomatosis in nongynecologic malignancy. A prospective study of prognostic factors. Cancer. 1989; 63:364-67.

2. Sadeghi B, Arvieux C, Glehen O, Beaujard AC, Rivoire M, Baulieux J, Fontaumard E, Brachet A, Caillot JL, Faure JL, Porcheron J, Peix JL, François Y, et al. Peritoneal carcinomatosis from non-gynecologic malignancies: results of the EVOCAPE 1 multicentric prospective study. Cancer. 2000; 88:358-63.

3. Jayne DG, Fook S, Loi C, Seow-Choen F. Peritoneal carcinomatosis from colorectal cancer. Br J Surg. 2002; 89:1545-50.

4. de Gramont A, Figer A, Seymour M, Homerin M, Hmissi A, Cassidy J, Boni C, Cortes-Funes H, Cervantes A, Freyer G, Papamichael D, Le Bail N, Louvet C, et al. Leucovorin and fluorouracil with or without oxaliplatin as first-line treatment in advanced colorectal cancer. J Clin Oncol. 2000; 18:2938-47.

5. Adachi $\mathrm{T}$, Hinoi $\mathrm{T}$, Egi $\mathrm{H}$, Shimomura $\mathrm{M}$, Ohdan $\mathrm{H}$. Oxaliplatin and molecular-targeted drug therapies improved the overall survival in colorectal cancer patients with synchronous peritoneal carcinomatosis undergoing incomplete cytoreductive surgery. Surg Today. 2015; 45:986-92.

6. Franko J, Shi Q, Goldman CD, Pockaj BA, Nelson GD, Goldberg RM, Pitot HC, Grothey A, Alberts SR, Sargent DJ. Treatment of colorectal peritoneal carcinomatosis with systemic chemotherapy: a pooled analysis of north central cancer treatment group phase III trials N9741 and N9841. J Clin Oncol. 2012; 30:263-67.

7. Kerscher AG, Chua TC, Gasser M, Maeder U, Kunzmann $\mathrm{V}$, Isbert C, Germer CT, Pelz JO. Impact of peritoneal carcinomatosis in the disease history of colorectal cancer management: a longitudinal experience of 2406 patients over two decades. Br J Cancer. 2013; 108:1432-39.

8. Sugarbaker PH, Gianola FJ, Speyer JC, Wesley R, Barofsky I, Meyers CE. Prospective, randomized trial of intravenous versus intraperitoneal 5-fluorouracil in patients with advanced primary colon or rectal cancer. Surgery. 1985; 98:414-22.

9. Sugarbaker PH. Peritonectomy procedures. Ann Surg. 1995; 221:29-42.

10. Jacquet P, Averbach AM, Stephens AD, Sugarbaker PH. Cancer recurrence following laparoscopic colectomy. Report of two patients treated with heated intraperitoneal chemotherapy. Dis Colon Rectum. 1995; 38:1110-14.

11. Sugarbaker PH, Cunliffe WJ, Belliveau J, de Bruijn EA, Graves T, Mullins RE, Schlag P. Rationale for integrating early postoperative intraperitoneal chemotherapy into the surgical treatment of gastrointestinal cancer. Semin Oncol. $1989 ; 16: 83-97$.

12. Verwaal VJ, van Ruth S, de Bree E, van Sloothen GW, van Tinteren H, Boot H, Zoetmulder FA. Randomized trial of cytoreduction and hyperthermic intraperitoneal chemotherapy versus systemic chemotherapy and palliative surgery in patients with peritoneal carcinomatosis of colorectal cancer. J Clin Oncol. 2003; 21:3737-43.

13. Glehen O, Kwiatkowski F, Sugarbaker PH, Elias D, Levine EA, De Simone M, Barone R, Yonemura Y, Cavaliere F, Quenet F, Gutman M, Tentes AA, Lorimier G, et al. Cytoreductive surgery combined with perioperative intraperitoneal chemotherapy for the management of peritoneal carcinomatosis from colorectal cancer: a multiinstitutional study. J Clin Oncol. 2004; 22:3284-92.

14. Elias D, Gilly F, Boutitie F, Quenet F, Bereder JM, Mansvelt B, Lorimier G, Dubè P, Glehen O. Peritoneal colorectal carcinomatosis treated with surgery and perioperative intraperitoneal chemotherapy: retrospective analysis of 523 patients from a multicentric French study. J Clin Oncol. 2010; 28:63-68.

15. Chua TC, Morris DL, Saxena A, Esquivel J, Liauw W, Doerfer J, Germer CT, Kerscher AG, Pelz JO. Influence of modern systemic therapies as adjunct to cytoreduction and perioperative intraperitoneal chemotherapy for patients with colorectal peritoneal carcinomatosis: a multicenter study. Ann Surg Oncol. 2011; 18:1560-67.

16. Glehen O, Cotte E, Schreiber V, Sayag-Beaujard AC, Vignal J, Gilly FN. Intraperitoneal chemohyperthermia and attempted cytoreductive surgery in patients with peritoneal carcinomatosis of colorectal origin. Br J Surg. 2004; 91:747-54.

17. Elias D, Delperro JR, Sideris L, Benhamou E, Pocard M, Baton O, Giovannini M, Lasser P. Treatment of peritoneal carcinomatosis from colorectal cancer: impact of complete cytoreductive surgery and difficulties in conducting randomized trials. Ann Surg Oncol. 2004; 11:518-21.

18. Elias D, Raynard B, Farkhondeh F, Goéré D, Rouquie D, Ciuchendea R, Pocard M, Ducreux M. Peritoneal 
carcinomatosis of colorectal origin. Gastroenterol Clin Biol. 2006; 30:1200-04.

19. Verwaal VJ, van Ruth S, Witkamp A, Boot H, van Slooten G, Zoetmulder FA. Long-term survival of peritoneal carcinomatosis of colorectal origin. Ann Surg Oncol. 2005; 12:65-71.

20. Shen P, Hawksworth J, Lovato J, Loggie BW, Geisinger KR, Fleming RA, Levine EA. Cytoreductive surgery and intraperitoneal hyperthermic chemotherapy with mitomycin C for peritoneal carcinomatosis from nonappendiceal colorectal carcinoma. Ann Surg Oncol. 2004; 11:178-86.

21. Passot G, Vaudoyer D, Cotte E, You B, Isaac S, Noël Gilly F, Mohamed F, Glehen O. Progression following neoadjuvant systemic chemotherapy may not be a contraindication to a curative approach for colorectal carcinomatosis. Ann Surg. 2012; 256:125-29.

22. Haslinger M, Francescutti V, Attwood K, McCart JA, Fakih M, Kane JM 3rd, Skitzki JJ. A contemporary analysis of morbidity and outcomes in cytoreduction/hyperthermic intraperitoneal chemoperfusion. Cancer Med. 2013; 2:334 42.

23. Turrini O, Lambaudie E, Faucher M, Viret F, Blache JL, Houvenaeghel G, Delpero JR. Initial experience with hyperthermic intraperitoneal chemotherapy. Arch Surg. 2012; 147:919-23.

24. Verwaal VJ, Bruin S, Boot H, van Slooten G, van Tinteren H. 8-year follow-up of randomized trial: cytoreduction and hyperthermic intraperitoneal chemotherapy versus systemic chemotherapy in patients with peritoneal carcinomatosis of colorectal cancer. Ann Surg Oncol. 2008; 15:2426-32.

25. Chua TC, Liauw W, Saxena A, Al-Mohaimeed K, Fransi S, Zhao J, Morris DL. Evolution of locoregional treatment for peritoneal carcinomatosis: single-center experience of 308 procedures of cytoreductive surgery and perioperative intraperitoneal chemotherapy. Am J Surg. 2011; 201:14956.

26. Chua TC, Liauw W, Zhao J, Morris DL. Comparative analysis of perioperative intraperitoneal chemotherapy regimen in appendiceal and colorectal peritoneal carcinomatosis. Int J Clin Oncol. 2013; 18:439-46.

27. Cavaliere F, De Simone M, Virzì S, Deraco M, Rossi CR, Garofalo A, Di Filippo F, Giannarelli D, Vaira M, Valle M, Pilati P, Perri P, La Pinta M, et al. Prognostic factors and oncologic outcome in 146 patients with colorectal peritoneal carcinomatosis treated with cytoreductive surgery combined with hyperthermic intraperitoneal chemotherapy: italian multicenter study S.I.T.I.L.O. Eur J Surg Oncol. 2011; $37: 148-54$.

28. Elias D, Lefevre JH, Chevalier J, Brouquet A, Marchal F, Classe JM, Ferron G, Guilloit JM, Meeus P, Goéré D, Bonastre J. Complete cytoreductive surgery plus intraperitoneal chemohyperthermia with oxaliplatin for peritoneal carcinomatosis of colorectal origin. J Clin Oncol. $2009 ; 27: 681-85$.
29. Esquivel J, Lowy AM, Markman M, Chua T, Pelz J, Baratti D, Baumgartner JM, Berri R, Bretcha-Boix P, Deraco M, Flores-Ayala G, Glehen O, Gomez-Portilla A, et al. The American Society of Peritoneal Surface Malignancies (ASPSM) Multiinstitution Evaluation of the Peritoneal Surface Disease Severity Score (PSDSS) in 1,013 Patients with Colorectal Cancer with Peritoneal Carcinomatosis. Ann Surg Oncol. 2014; 21:4195-201.

30. Cao C, Yan TD, Black D, Morris DL. A systematic review and meta-analysis of cytoreductive surgery with perioperative intraperitoneal chemotherapy for peritoneal carcinomatosis of colorectal origin. Ann Surg Oncol. 2009; 16:2152-65.

31. Esquivel J, Sticca R, Sugarbaker P, Levine E, Yan TD, Alexander R, Baratti D, Bartlett D, Barone R, Barrios P, Bieligk S, Bretcha-Boix P, Chang CK, et al, and Society of Surgical Oncology Annual Meeting. Cytoreductive surgery and hyperthermic intraperitoneal chemotherapy in the management of peritoneal surface malignancies of colonic origin: a consensus statement. Ann Surg Oncol. 2007; 14:128-33.

32. Yonemura Y, Canbay E, Ishibashi H. Prognostic factors of peritoneal metastasis from colorectal cancer following cytoreductive surgery and perioperative chemotherapy. Scien-tificWorldJournal. 2013; 2013:978394.

33. Li Y, Yu Y, Liu Y. Report on the 9th international congress on peritoneal surface malig-nancies. Cancer Biol Med. 2014; 11:281-84.

34. Chua TC, Pelz JO, Kerscher A, Morris DL, Esquivel J. Critical analysis of 33 patients with peritoneal carcinomatosis secondary to colorectal and appendiceal signet ring cell carcinoma. Ann Surg Oncol. 2009; 16:276570.

35. Elias D, Blot F, El Otmany A, Antoun S, Lasser P, Boige V, Rougier P, Ducreux M. Curative treatment of peritoneal carcinomatosis arising from colorectal cancer by complete resection and intraperitoneal chemotherapy. Cancer. 2001; 92:71-76.

36. Elias D, Benizri E, Di Pietrantonio D, Menegon P, Malka D, Raynard B. Comparison of two kinds of intraperitoneal chemotherapy following complete cytoreductive surgery of colorectal peritoneal carcinomatosis. Ann Surg Oncol. 2007; 14:509-14.

37. Franko J, Ibrahim Z, Gusani NJ, Holtzman MP, Bartlett DL, Zeh HJ 3rd. Cytoreductive surgery and hyperthermic intraperitoneal chemoperfusion versus systemic chemotherapy alone for colorectal peritoneal carcinomatosis. Cancer. 2010; 116:3756-62.

38. Gervais MK, Dubé P, McConnell Y, Drolet P, Mitchell A, Sideris L. Cytoreductive surgery plus hyperthermic intraperitoneal chemotherapy with oxaliplatin for peritoneal carcinomatosis arising from colorectal cancer. J Surg Oncol. $2013 ; 108: 438-43$.

39. Huang CQ, Feng JP, Yang XJ, Li Y. Cytoreductive surgery plus hyperthermic intraperitoneal chemotherapy improves 
survival of patients with peritoneal carcinomatosis from colorectal cancer: a case-control study from a Chinese center. J Surg Oncol. 2014; 109:730-39.

40. Passot G, You B, Boschetti G, Fontaine J, Isaac S, Decullier E, Maurice C, Vaudoyer D, Gilly FN, Cotte E, Glehen O. Pathological response to neoadjuvant chemotherapy: a new prognosis tool for the curative management of peritoneal colorectal carcinomatosis. Ann Surg Oncol. 2014; 21:260814.

41. Alzahrani N, Ferguson JS, Valle SJ, Liauw W, Chua T, Morris DL. Cytoreductive surgery and hyperthermic intraperitoneal chemotherapy: long-term results at $\mathrm{St}$ George Hospital, Aus-tralia. ANZ J Surg. Epub ahead of print. https://doi.org/10.1111/ans.13152.. Published July 14, 2015.

42. Beaujard AC, Glehen O, Caillot JL, Francois Y, Bienvenu J, Panteix G, Garbit F, Grandclément E, Vignal J, Gilly FN. Intraperitoneal chemohyperthermia with mitomycin $\mathrm{C}$ for digestive tract cancer patients with peritoneal carcinomatosis. Cancer. 2000; 88:2512-19.

43. Bijelic L, Yan TD, Sugarbaker PH. Treatment failure following complete cytoreductive surgery and perioperative intraperitoneal chemotherapy for peritoneal dissemination from colorectal or appendiceal mucinous neoplasms. J Surg Oncol. 2008; 98:295-99.

44. Braam HJ, van Oudheusden TR, de Hingh IH, Nienhuijs SW, Boerma D, Wiezer MJ, van Ramshorst B. Patterns of recurrence following complete cytoreductive surgery and hyperthermic intraperitoneal chemotherapy in patients with peritoneal carcinomatosis of colorectal cancer. J Surg Oncol. 2014; 109:841-47.

45. Cao CQ, Yan TD, Liauw W, Morris DL. Comparison of optimally resected hepatectomy and peritonectomy patients with colorectal cancer metastasis. J Surg Oncol. 2009; 100:529-33.

46. Cavaliere F, Valle M, De Simone M, Deraco M, Rossi CR, Di Filippo F, Verzi S, Giannarelli D, Perri P, Pilati PL, Vaira M, Di Filippo S, Garofalo A. 120 peritoneal carcinomatoses from colorectal cancer treated with peritonectomy and intra-abdominal chemohyperthermia: a S.I.T.I.L.O. multicentric study. In Vivo. 2006; 20:747-50.

47. Ceelen W, Van Nieuwenhove Y, Putte DV, Pattyn P. Neoadjuvant chemotherapy with bevacizumab may improve outcome after cytoreduction and hyperthermic intraperitoneal chemoperfusion (HIPEC) for colorectal carcinomatosis. Ann Surg Oncol. 2014; 21:3023-28.

48. Desantis M, Bernard JL, Casanova V, Cegarra-Escolano M, Benizri E, Rahili AM, Benchimol D, Bereder JM. Morbidity, mortality, and oncological outcomes of 401 consecutive cytoreductive procedures with hyperthermic intraperitoneal chemotherapy (HIPEC). Langenbecks Arch Surg. 2015; 400:37-48.

49. Elias D, Pocard M, Sideris L, Edè C, Ducreux M, Boige V, Lasser P. Preliminary results of intraperitoneal chemohyperthermia with oxaliplatin in peritoneal carcinomatosis of colorectal origin. Br J Surg. 2004; 91:455-56.

50. Elias D, Faron M, Goéré D, Dumont F, Honoré C, Boige V, Malka D, Ducreux M. A simple tumor load-based nomogram for surgery in patients with colorectal liver and peritoneal metastases. Ann Surg Oncol. 2014; 21:2052-58.

51. Evers DJ, Verwaal VJ. Indication for oophorectomy during cytoreduction for intraperitoneal metastatic spread of colorectal or appendiceal origin. Br J Surg. 2011; 98:28792.

52. Franko J, Gusani NJ, Holtzman MP, Ahrendt SA, Jones HL, Zeh HJ 3rd, Bartlett DL. Multivisceral resection does not affect morbidity and survival after cytoreductive surgery and chemoperfusion for carcinomatosis from colorectal cancer. Ann Surg Oncol. 2008; 15:3065-72.

53. Glehen O, Mithieux F, Osinsky D, Beaujard AC, Freyer G, Guertsch P, Francois Y, Peyrat P, Panteix G, Vignal J, Gilly FN. Surgery combined with peritonectomy procedures and intraperitoneal chemohyperthermia in abdominal cancers with peritoneal carcinomatosis: a phase II study. J Clin Oncol. 2003; 21:799-806.

54. Glehen O, Gilly FN, Boutitie F, Bereder JM, Quenet F, Sideris L, Mansvelt B, Lorimier G, Msika S, Elias D, and French Surgical Association. Toward curative treatment of peritoneal carcinomatosis from nonovarian origin by cytoreductive surgery combined with perioperative intraperitoneal chemotherapy: a multi-institutional study of 1,290 patients. Cancer. 2010; 116:5608-18.

55. Gomes da Silva R, Cabanas J, Sugarbaker PH. Limited survival in the treatment of carcinomatosis from rectal cancer. Dis Colon Rectum. 2005; 48:2258-63.

56. Gusani NJ, Cho SW, Colovos C, Seo S, Franko J, Richard SD, Edwards RP, Brown CK, Holtzman MP, Zeh HJ, Bartlett DL. Aggressive surgical management of peritoneal carcinomatosis with low mortality in a high-volume tertiary cancer center. Ann Surg Oncol. 2008; 15:754-63.

57. Hamilton T, Lanuke K, Mack LA, Temple WJ. Long-term follow-up in the treatment of peritoneal carcinomatosis. Am J Surg. 2011; 201:650-54.

58. Hompes D, D'Hoore A, Van Cutsem E, Fieuws S, Ceelen W, Peeters M, Van der Speeten K, Bertrand C, Legendre $\mathrm{H}$, Kerger J. The treatment of peritoneal carcinomatosis of colorectal cancer with complete cytoreductive surgery and hyperthermic intraperitoneal peroperative chemotherapy (HIPEC) with oxaliplatin: a Belgian multicentre prospective phase II clinical study. Ann Surg Oncol. 2012; 19:2186-94.

59. Hompes D, D'Hoore A, Wolthuis A, Fieuws S, Mirck B, Bruin S, Verwaal V. The use of Oxaliplatin or Mitomycin $\mathrm{C}$ in HIPEC treatment for peritoneal carcinomatosis from colorectal cancer: a comparative study. J Surg Oncol. 2014; 109:527-32.

60. Iversen LH, Rasmussen PC, Hagemann-Madsen R, Laurberg S. Cytoreductive surgery and hyperthermic intraperitoneal chemotherapy for peritoneal carcinomatosis: 
the Danish experience. Colorectal Dis. 2013; 15:e365-72.

61. Kecmanovic DM, Pavlov MJ, Ceranic MS, Sepetkovski AV, Kovacevic PA, Stamenkovic AB. Treatment of peritoneal carcinomatosis from colorectal cancer by cytoreductive surgery and hyperthermic perioperative intraperitoneal chemotherapy. Eur J Surg Oncol. 2005; 31:147-52.

62. Kianmanesh R, Scaringi S, Sabate JM, Castel B, PonsKerjean N, Coffin B, Hay JM, Flamant Y, Msika S. Iterative cytoreductive surgery associated with hyperthermic intraperitoneal chemotherapy for treatment of peritoneal carcinomatosis of colorectal origin with or without liver metastases. Ann Surg. 2007; 245:597-603.

63. Klaver YL, de Hingh IH, Boot H, Verwaal VJ. Results of cytoreductive surgery and hyperthermic intraperitoneal chemotherapy after early failure of adjuvant systemic chemotherapy. J Surg Oncol. 2011; 103:431-34.

64. Klaver YL, Chua TC, de Hingh IH, Morris DL. Outcomes of elderly patients undergoing cytoreductive surgery and perioperative intraperitoneal chemotherapy for colorectal cancer peritoneal carcinomatosis. J Surg Oncol. 2012; 105:113-18.

65. Kuijpers AM, Mirck B, Aalbers AG, Nienhuijs SW, de Hingh IH, Wiezer MJ, van Ramshorst B, van Ginkel RJ, Havenga K, Bremers AJ, de Wilt JH, Te Velde EA, Verwaal VJ. Cytoreduction and HIPEC in the Netherlands: nationwide long-term outcome following the Dutch protocol. Ann Surg Oncol. 2013; 20:4224-30.

66. Kuijpers AM, Mehta AM, Boot H, van Leerdam ME, Hauptmann M, Aalbers AG, Verwaal VJ. Perioperative systemic chemotherapy in peritoneal carcinomatosis of lymph node positive colorectal cancer treated with cytoreductive surgery and hyperthermic intraperitoneal chemotherapy. Ann Oncol. 2014; 25:864-69.

67. Lanuke K, Mack LA, Temple WJ. Phase II study of regional treatment for peritoneal carcinomatosis. Am J Surg. 2009; 197:614-18.

68. Levine EA, Stewart JH 4th, Shen P, Russell GB, Loggie BL, Votanopoulos KI. Intraperitoneal chemotherapy for peritoneal surface malignancy: experience with 1,000 patients. J Am Coll Surg. 2014; 218:573-85.

69. McConnell YJ, Mack LA, Francis WP, Ho T, Temple WJ. HIPEC+EPIC versus HIPEC-alone: differences in major complications following cytoreduction surgery for peritoneal malignancy. J Surg Oncol. 2013; 107:591-96.

70. Nikolic S, Dzodic R, Zegarac M, Djurisic I, Gavrilovic D, Vojinovic V, Kocic M, Santrac N, Radlovic P, Radosavljevic D, Pupic G, Martinovic A. Survival prognostic factors in patients with colorectal peritoneal carcinomatosis treated with cytoreductive surgery and intraoperative hyperthermic intraperitoneal chemotherapy: a single institution experience. J BUON. 2014; 19:66-74.

71. Pilati P, Mocellin S, Rossi CR, Foletto M, Campana L, Nitti D, Lise M. Cytoreductive surgery combined with hyperthermic intraperitoneal intraoperative chemotherapy for peritoneal carcinomatosis arising from colon adenocarcinoma. Ann Surg Oncol. 2003; 10:508-13.

72. Prada-Villaverde A, Esquivel J, Lowy AM, Markman M, Chua T, Pelz J, Baratti D, Baumgartner JM, Berri R, Bretcha-Boix P, Deraco M, Flores-Ayala G, Glehen O, et al. The American Society of Peritoneal Surface Malignancies evaluation of HIPEC with Mitomycin C versus Oxaliplatin in 539 patients with colon cancer undergoing a complete cytoreductive surgery. J Surg Oncol. 2014; 110:779-85.

73. Quenet F, Goéré D, Mehta SS, Roca L, Dumont F, Hessissen M, Saint-Aubert B, Elias D. Results of two bi-institutional prospective studies using intraperitoneal oxaliplatin with or without irinotecan during HIPEC after cytoreductive surgery for colorectal carcinomatosis. Ann Surg. 2011; 254:294-301.

74. Rivard JD, McConnell YJ, Temple WJ, Mack LA. Cytoreduction and heated intraperitoneal chemotherapy for colorectal cancer: are we excluding patients who may benefit? J Surg Oncol. 2014; 109:104-09.

75. Rodt AP, Svarrer RO, Iversen LH. Clinical course for patients with peritoneal carcinomatosis excluded from cytoreductive surgery and hyperthermic intraperitoneal chemotherapy. World J Surg Oncol. 2013; 11:232.

76. Shen P, Thai K, Stewart JH, Howerton R, Loggie BW, Russell GB, Levine EA. Peritoneal surface disease from colorectal cancer: comparison with the hepatic metastases surgical paradigm in optimally resected patients. Ann Surg Oncol. 2008; 15:3422-32.

77. Swellengrebel HA, Zoetmulder FA, Smeenk RM, Antonini N, Verwaal VJ. Quantitative intra-operative assessment of peritoneal carcinomatosis - a comparison of three prognostic tools. Eur J Surg Oncol. 2009; 35:1078-84.

78. Tabrizian P, Shrager B, Jibara G, Yang MJ, Romanoff A, Hiotis S, Sarpel U, Labow DM. Cytoreductive surgery and hyperthermic intraperitoneal chemotherapy for peritoneal carcinomatosis: outcomes from a single tertiary institution. J Gastrointest Surg. 2014; 18:1024-31.

79. Teo MC, Tan GH, Tham CK, Lim C, Soo KC. Cytoreductive surgery and hyperthermic intraperitoneal chemotherapy in Asian patients: 100 consecutive patients in a single institution. Ann Surg Oncol. 2013; 20:2968-74.

80. Teo MC, Ching Tan GH, Lim C, Chia CS, Tham CK, Soo KC. Colorectal peritoneal carcinomatosis treated with cytoreductive surgery and hyperthermic intraperitoneal chemotherapy: the experience of a tertiary Asian center. Asian J Surg. 2015; 38:65-73.

81. Ung L, Chua TC, David L M. Peritoneal metastases of lower gastrointestinal tract origin:a comparative study of patient outcomes following cytoreduction and intraperitoneal chemotherapy. J Cancer Res Clin Oncol. 2013; 139:1899908.

82. Vaira M, Cioppa T, D’Amico S, de Marco G, D'Alessandro M, Fiorentini G, De Simone M. Treatment of peritoneal 
carcinomatosis from colonic cancer by cytoreduction, peritonectomy and hyperthermic intraperitoneal chemotherapy (HIPEC). Experience of ten years. In Vivo. 2010; 24:79-84.

83. van Leeuwen BL, Graf W, Pahlman L, Mahteme H. Swedish experience with peritonectomy and HIPEC. HIPEC in peritoneal carcinomatosis. Ann Surg Oncol. 2008; 15:745-53.

84. van Oudheusden TR, Braam HJ, Nienhuijs SW, Wiezer MJ, van Ramshorst B, Luyer MD, Lemmens VE, de Hingh IH. Cytoreduction and hyperthermic intraperitoneal chemotherapy: a feasible and effective option for colorectal cancer patients after emergency surgery in the presence of peritoneal carcinomatosis. Ann Surg Oncol. 2014; 21:262126.

85. van Oudheusden TR, Braam HJ, Nienhuijs SW, Wiezer MJ, van Ramshorst B, Luyer P, de Hingh IH. Poor outcome after cytoreductive surgery and HIPEC for colorectal peritoneal carcinomatosis with signet ring cell histology. J Surg Oncol. 2015; 111:237-42.

86. Varban O, Levine EA, Stewart JH, McCoy TP, Shen P. Outcomes associated with cytoreductive surgery and intraperitoneal hyperthermic chemotherapy in colorectal cancer patients with peritoneal surface disease and hepatic metastases. Cancer. 2009; 115:3427-36.

87. Votanopoulos KI, Swett K, Blackham AU, Ihemelandu C, Shen P, Stewart JH, Levine EA. Cytoreductive surgery with hyperthermic intraperitoneal chemotherapy in peritoneal carcinomatosis from rectal cancer. Ann Surg Oncol. 2013; 20:1088-92.

88. Winer J, Zenati M, Ramalingam L, Jones H, Zureikat A, Holtzman M, Lee K, Ahrendt S, Pingpank J, Zeh HJ, Bartlett DL, Choudry HA. Impact of aggressive histology and location of primary tumor on the efficacy of surgical therapy for peritoneal carcinomatosis of colorectal origin. Ann Surg Oncol. 2014; 21:1456-62.

89. Witkamp AJ, de Bree E, Kaag MM, Boot H, Beijnen JH, van Slooten GW, van Coevorden F, Zoetmulder FA. Extensive cytoreductive surgery followed by intraoperative hyperthermic intraperitoneal chemotherapy with mitomycin-C in patients with peritoneal carcinomatosis of colorectal origin. Eur J Cancer. 2001; 37:979-84.

90. Yan TD, Chu F, Links M, Kam PC, Glenn D, Morris DL. Cytoreductive surgery and perioperative intraperitoneal chemotherapy for peritoneal carcinomatosis from colorectal carcinoma: non-mucinous tumour associated with an improved survival. Eur J Surg Oncol. 2006; 32:1119-24.

91. Yan TD, Morris DL. Cytoreductive surgery and perioperative intraperitoneal chemotherapy for isolated colorectal peritoneal carcinomatosis: experimental therapy or standard of care? Ann Surg. 2008; 248:829-35.

92. Zanon C, Bortolini M, Chiappino I, Simone P, Bruno F, Gaglia P, Airoldi M, Deriu L, Mashiah A. Cytoreductive surgery combined with intraperitoneal chemohyperthermia for the treatment of advanced colon cancer. World J Surg.
2006; 30:2025-32.

93. Dimick JB, Cowan JA Jr, Upchurch GR Jr, Colletti LM. Hospital volume and surgical outcomes for elderly patients with colorectal cancer in the United States. J Surg Res. 2003; 114:50-56.

94. Pal N, Axisa B, Yusof S, Newcombe RG, Wemyss-Holden $\mathrm{S}$, Rhodes M, Lewis MP. Volume and outcome for major upper GI surgery in England. J Gastrointest Surg. 2008; 12:353-57.

95. Ghaferi AA, Birkmeyer JD, Dimick JB. Variation in hospital mortality associated with inpatient surgery. N Engl J Med. 2009; 361:1368-75.

96. Noordzij PG, Poldermans D, Schouten O, Bax JJ, Schreiner FA, Boersma E. Postoperative mortality in The Netherlands: a population-based analysis of surgery-specific risk in adults. Anesthesiology. 2010; 112:1105-15.

97. Jakobson T, Karjagin J, Vipp L, Padar M, Parik AH, Starkopf L, Kern H, Tammik O, Starkopf J. Postoperative complications and mortality after major gastrointestinal surgery. Medicina (Kaunas). 2014; 50:111-17.

98. Chua TC, Yan TD, Saxena A, Morris DL. Should the treatment of peritoneal carcinomatosis by cytoreductive surgery and hyperthermic intraperitoneal chemotherapy still be regarded as a highly morbid procedure?: a systematic review of morbidity and mortality. Ann Surg. 2009; 249:900-07.

99. Goéré D, Souadka A, Faron M, Cloutier AS, Viana B, Honoré C, Dumont F, Elias D. Extent of colorectal peritoneal carcinomatosis: attempt to define a threshold above which HIPEC does not offer survival benefit: a comparative study. Ann Surg Oncol. 2015; 22:2958-64.

100. Faron M, Macovei R, Goéré D, Honoré C, Benhaim L, Elias D. Linear Relationship of Peritoneal Cancer Index and Survival in Patients with Peritoneal Metastases from Colorectal Cancer. Ann Surg Oncol. 2016; 23:114-19.

101. Maillet M, Glehen O, Lambert J, Goere D, Pocard M, Msika S, Passot G, Elias D, Eveno C, Sabaté JM, Lourenco N, André T, Gornet JM, and BIG-RENAPE Working Group. Early Postoperative Chemotherapy After Complete Cytoreduction and Hyperthermic Intraperitoneal Chemotherapy for Isolated Peritoneal Carcinomatosis of Colon Cancer: A Multicenter Study. Ann Surg Oncol. 2016; 23:863-69.

102. Simkens GA, van Oudheusden TR, Luyer MD, Nienhuijs SW, Nieuwenhuijzen GA, Rutten HJ, de Hingh IH. Serious Postoperative Complications Affect Early Recurrence After Cytoreductive Surgery and HIPEC for Colorectal Peritoneal Carcinomatosis. Ann Surg Oncol. 2015; 22:2656-62.

103. Frøysnes IS, Larsen SG, Spasojevic M, Dueland S, Flatmark K. Complete cytoreductive surgery and hyperthermic intraperitoneal chemotherapy for colorectal peritoneal metastasis in Norway: prognostic factors and oncologic outcome in a national patient cohort. J Surg Oncol. 2016; 114:222-27. 
104. Passot G, Vaudoyer D, Villeneuve L, Kepenekian V, Beaujard AC, Bakrin N, Cotte E, Gilly FN, Glehen O. What made hyperthermic intraperitoneal chemotherapy an effective curative treatment for peritoneal surface malignancy: A 25 -year experience with 1,125 procedures. J Surg Oncol. 2016; 113:796-803.

105. North of England Antidepressant Guideline Development Group. Evidence Based Guideline for the choice of antidepressants for depression in primary care. Newcastle upon Tyne: Centre for Health Services Research; 1997.

106. Eccles M, Rousseau N, Freemantle N. Updating evidencebased clinical guidelines. J Health Serv Res Policy. 2002; 7:98-103.

107. National Cancer Institute. Common Terminology Criteria for Adverse Events version 4.0. Available: http://ctep. cancer.gov/protocolDevelopment/electronic_applications/ ctc.htm. [Accessed January 11, 2016]
108. Tierney JF, Stewart LA, Ghersi D, Burdett S, Sydes MR. Practical methods for incorporating summary time-to-event data into meta-analysis. Trials. 2007; 8:16.

109. Higgins JP, Thompson SG, Deeks JJ, Altman DG. Measuring inconsistency in meta-analyses. BMJ. 2003; 327:557-60.

110. Higgins JP, Thompson SG. Quantifying heterogeneity in a meta-analysis. Stat Med. 2002; 21:1539-58.

111. Egger M, Davey Smith G, Schneider M, Minder C. Bias in meta-analysis detected by a simple, graphical test. BMJ. 1997; 315:629-34.

112. Begg CB, Mazumdar M. Operating characteristics of a rank correlation test for publication bias. Biometrics. 1994; 50:1088-101. 